\title{
Optimal Scheduling of Random Flexible Demands in Smart Grid
}

\author{
by \\ Mohammad Mahdi Karbasioun
}

\author{
A dissertation submitted to the \\ Faculty of Graduate and Postdoctoral Affairs \\ in partial fulfillment of the requirements for the degree of
}

Doctor of Philosophy in Electrical and Computer Engineering

Ottawa-Carleton Institute for Electrical and Computer Engineering

Department of Systems and Computer Engineering

Carleton University

Ottawa, Ontario

February, 2018

(C) Copyright

Mohammad Mahdi Karbasioun, 2018 
The undersigned hereby recommends to the

Faculty of Graduate and Postdoctoral Affairs acceptance of the dissertation

\title{
Optimal Scheduling of Random Flexible Demands in Smart Grid
}

\author{
submitted by Mohammad Mahdi Karbasioun \\ in partial fulfillment of the requirements for the degree of \\ Doctor of Philosophy in Electrical and Computer Engineering \\ Professor Petros Spachos, External Examiner \\ Professor Ioannis Lambadaris, Thesis Supervisor \\ Professor Evangelos Kranakis, Thesis Co-supervisor \\ Professor Yvan Labiche, Chair, \\ Department of Systems and Computer Engineering \\ Ottawa-Carleton Institute for Electrical and Computer Engineering \\ Department of Systems and Computer Engineering \\ Carleton University \\ February, 2018
}




\section{Abstract}

On the consumer side of the electrical grid, there are some demands with some kind of "flexibility" in nature. Wisely exploiting these flexibilities could result in better utilization of the available resources.

Inspired by the existence of electric appliances with flexibility on their charging rate, we study the problem of optimally serving a set of "Malleable RectangularShape" energy requirements in a finite time interval. That is each demand must be supplied continuously with a constant intensity and duration bounded by left- and right-malleability constraints. At each moment of time, the total power consumption of the grid is the sum of all the consumption rates of the demands being supplied at that moment. First, we assume that the malleability constraints are the same for all demands. Then, we identify the lower bound on the optimal value of the power peak. We extend our study by considering stochastic malleability constraints, where each demand has its own constraint pair. In this case, we also include a convex cost of power consumption in our treatment. In each case, we propose an on-line scheduling algorithm, which is asymptotically optimal with respect to the given cost criteria.

As another type of flexible demands, we address the problem of controlling a charging station of Plug-in Hybrid Electric Vehicles (PHEVs), assuming they can tolerate rejection of their energy request. We introduce a model for a charging station, in which PHEVs are charged either directly from the electrical grid or from a local storage unit deployed for each class of customers. The control actions to be determined by the charging station operator are as follows: the probability of blocking new arrivals, the rate of charging each local storage unit, the proportion of cars being served by each local storage unit and eventually the proportion of the available power from the grid to be assigned to each class of customers. We will show how to find a control policy minimizing the utilization cost of the charging station. 


$$
\begin{aligned}
& \text { To my wonderful wife, Bahareh. } \\
& \text { for her endless support, patience and love, } \\
& \text { To my lovely Sha, } \\
& \text { To my great Wother and Father, } \\
& \text { for their enormous helfs and endless sacrificess } \\
& \text { And } \\
& \text { \%o my beloved family. }
\end{aligned}
$$




\section{Acknowledgments}

Firstly, I would like to express my appreciation and gratitude to Professor Ioannis Lambadaris and Professor Evangelos Kranakis for their priceless support, patience and supervision. This dissertation would not have been achievable without their help and guidance.

Besides, I am sincerely thankful to Professor Gennady Shaikhet for his precious time and for all the valuable suggestions and motivations and comments offered throughout this work.

I would like to thank my love, Bahar, for her endless patience and continuous encouragement throughout these years. This accomplishment would not have been possible without her constant help and support. I would also like to express my deepest gratitude to my wonderful parents for their love, support, and self-sacrifices. Passing through this long road was not possible without the energy and motivation I have received throughout these years from my wife, my daughter, my parents and my beloved family and friend. 


\section{Table of Contents}

Abstract

Acknowledgments $\quad$ v

Table of Contents $\quad$ vi

List of Tables $\quad$ ix

List of Figures $\quad$ x

Nomenclature $\quad$ xiv

1 Introduction 1

1.1 Research Motivations . . . . . . . . . . . . . . . . 2

1.2 Research Overview and Contributions . . . . . . . . . . . . 5

1.2.1 Demand Side Management . . . . . . . . . . . . 5

1.2.2 Optimal Charging Station Strategy . . . . . . . . . . . . 6

1.2.3 Published and Submitted Papers . . . . . . . . . . . . . . 9

1.3 Thesis Organization . . . . . . . . . . . . . . . . . . 10

2 Background and Related Works 12

2.1 Demand Side Management . . . . . . . . . . . . . . . . . 13

2.2 scheduling of random malleable demands . . . . . . . . . . . . . 16

2.2.1 Strip Packingproblem and the related literature . . . . . . . . 16

2.3 Optimal Controlling of Charging Stations for Electrical Vehicles . . . 21

2.3.1 Markov Decision Processes . . . . . . . . . . . . . . . . 22

2.3.2 Related Literature . . . . . . . . . . . . . . . . . . . . 27

2.4 Summary . . . . . . . . . . . . . . . . . . . . 29 
3 Power Strip Packing of Malleable Demands 30

3.1 Model . . . . . . . . . . . . . . . . . . . . . . 30

3.2 Main results . . . . . . . . . . . . . . . . . . . . . 32

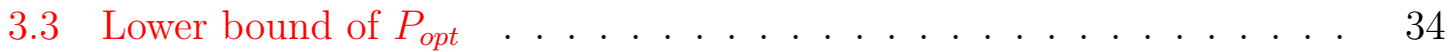

3.4 Algorithm . . . . . . . . . . . . . . . . . . . 38

3.4.1 Ideal Cases . . . . . . . . . . . . . . . . . . . . 38

3.4 .2 Near Ideal Cases . . . . . . . . . . . . . . . . . . . . . . 38

3.4 .3 Non-Ideal Cases . . . . . . . . . . . . . . . . . . . . . . 39

3.5 Proof of Theorem 1 . . . . . . . . . . . . . . . . 40

3.6 Computational Results . . . . . . . . . . . . . . . . . . 40

3.7 Summary . . . . . . . . . . . . . . . . . . . . 42

4 Asymptotic Convex Optimization for Packing Random Malleable Demands $\quad 43$

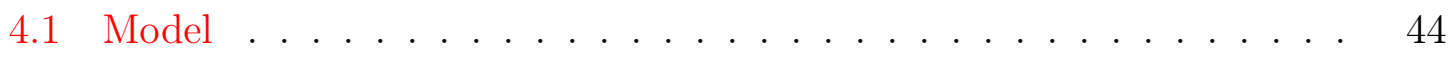

4.2 Cost Optimization. The Main Result . . . . . . . . . . . . . . . 46

4.2.1 Asymptotic Setting on a Grid . . . . . . . . . . . . . . . . . . 47

4.2.2 Optimal Policy . . . . . . . . . . . . . . . . 48

4.2.3 Discussion of the Algorithm . . . . . . . . . . . . . . . . . . 49

4.2.4 Proof of Theorem 2 . . . . . . . . . . . . . . 51

4.2.5 Generalization of the "uniformity" assumption . . . . . . . . . 55

4.2.6 Fixed Malleability Constraints . . . . . . . . . . . . 55

4.3 Computational Results for Covering Policy . . . . . . . . . . . . . . . 61

4.4 Sequential Application of Covering Policy . . . . . . . . . . . . . 68

4.5 Summary . . . . . . . . . . . . . . . . . . . . 72

5 Optimal Controlling of Charging Stations for Electrical Vehicles under Real Time Pricing $\quad 74$

5.1 Charging Station Model: Overall View . . . . . . . . . . . . . 74

5.2 Charging Station Model: Mathematical details . . . . . . . . . 76

5.2.1 System primitives . . . . . . . . . . . . 76

5.2.2 Introducing controls . . . . . . . . . . . . . . . . 79

5.3 Optimal Control Policy . . . . . . . . . . . . . . . . . 80

5.3.1 Deriving the Optimal Policy . . . . . . . . . . . . . . 82

5.3.2 Expected total $\beta$-discounted cost . . . . . . . . . . 88 
5.3.3 Minimizing the infinite horizon Average Cost . . . . . . . . 88

5.3.4 Numerical Examples . . . . . . . . . . . . . . . . . . . . . . . 89

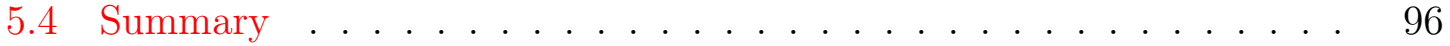

6 Optimal Controlling of Charging Stations for Heterogeneous Elec$\begin{array}{ll}\text { tric Vehicles } & 97\end{array}$

6.1 Charging Station Model: Overall View . . . . . . . . . . . . 97

6.2 Controlling the Charging Station with Multi-Class of customers . . . 98

6.2.1 Off-line splitting of Power from the Grid . . . . . . . . . . 102

6.2.2 Real-Time splitting of Power from the Grid . . . . . . . . . 104

6.2 .3 Numerical Examples . . . . . . . . . . . . . . . . . . . 105

6.3 Summary . . . . . . . . . . . . . . . . . . . 107

7 Conclusions and Suggestions for Future Research 109

7.1 Conclusions . . . . . . . . . . . . . . . . . . . . . . . . . . 109

7.2 Future Directions . . . . . . . . . . . . . . . . . . . . . . 111

$\begin{array}{ll}\text { List of References } & 113\end{array}$

$\begin{array}{lll}\text { Appendix A Proof of Lemma } 1 \text { in Chapter } 3 & 120\end{array}$

$\begin{array}{ll}\text { Appendix B Proof of Lemma } 2 \text { in Chapter } 3 & 121\end{array}$

Appendix C Proof of Lemma 4 in Chapter 3

Appendix D Proof of Lemma 5 in Chapter $3 \quad 123$ 


\section{List of Tables}

4.1 The summary of the Assumptions in Subsection 4.2.1 . . . . . . . . . 62

5.1 The summary of all Optimal Action Criteria . . . . . . . . . . . . 90 


\section{List of Figures}

1.1 A typical daily load curve in a grid. For example, this curve is computed based on the power consumption in France Power distribution network on February 4, 2016. . . . . . . . . . . . . . . . . 3

1.2 Electric Reliability Council of Texas (ERCOT) system load profile on August 27, 1990. This figure illustrates a typical commitment of generation assets. The reserve power supply is about $5.9 \mathrm{GW}$ during peak hours .......................... 4

2.1 Malleable rectangular Shape Energy Demand representation. . . . . . 17

2.2 Different interpretations of power and height in PSP and TSP. For simplicity the height of each rectangle is assumed to be 1. In PSP: $P\left(t_{1}\right)=5$ and $P\left(t_{2}\right)=3$ where in TSP $H\left(t_{1}\right)=H\left(t_{2}\right)=5 \ldots \ldots$

3.1 Different interpretations of power and height in PSP and TSP. For simplicity the height of each rectangle is assumed to be 1. In PSP: $P\left(t_{1}\right)=5$ and $P\left(t_{2}\right)=3$ where in TSP $H\left(t_{1}\right)=H\left(t_{2}\right)=5 \ldots \ldots$

3.2 Coverable region vs. Non-Coverable region. The Coverable region is shown with shadow. . . . . . . . . . . . . . . .

3.3 Performance of the algorithms for Near-Ideal case with respect to Peak criteria for different number of demands, where malleability constraint pair is in the coverable region $\mathcal{G}(\ell=0.35714$ and $r=0.75758)$, that is $Z^{*}=1 \ldots \ldots \ldots \ldots \ldots \ldots \ldots \ldots$

3.4 Performance of the algorithms for Non-Ideal case with respect to Peak criteria for different number of demands, where malleability constraint pair is NOT in the coverable region $\mathcal{G}(\ell=0.3571$ and $r=0.43103)$, that is $Z^{*}<1 \ldots \ldots \ldots \ldots \ldots \ldots \ldots \ldots$ 
4.1 An example of a Malleable Rectangular-Shape energy demand, with energy requirement $A_{i}$ and malleability constraints $\left(\ell_{i}, r_{i}\right)$. In this example, this energy demand is served in two different ways: $\left(\tau_{i}, s_{i}\right)$ and $\left(\tau_{i}, s_{i}^{\prime}\right)$. The service times $s_{i}$ and $s_{i}^{\prime}$ satisfy $0<\ell_{i} \leq s_{i}^{\prime}<s_{i} \leq r_{i} \leq 1$.

4.2 Applying the covering policy $\mathbf{v}^{*}$ for $d=8$ and $r_{i}=\frac{3}{8} \ldots \ldots$. . . .

4.3 Applying the covering policy $\mathbf{v}^{*}$ for scheduling the demands with different right malleability constraints $r_{i}$. Each sub-figure illustrates the scheduling of demands with their right malleability constraints equal to $\frac{k}{8}$, where $k=1, \cdots, d$, and $d=8 \ldots \ldots \ldots$

4.4 Performance of the covering policy $\mathbf{v}^{*}$ in Algorithm 2 with respect to both cost criteria: maximum power and the total convex cost of power consumption, where malleability constraint pairs are i.i.d random vectors in $\Omega_{d}$, with $d=3$ and $n \in\{500,1000,1500, \cdots, 10000\} . .$.

4.5 Performance of the covering policy $\mathbf{v}^{*}$ in Algorithm 2 with respect to both cost criteria: maximum power and the total convex cost of power consumption, where malleability constraint pairs are i.i.d random vectors in $\Omega_{d}$, with $d=9$ and $n \in\{500,1000,1500, \cdots, 10000\}$. . . . .

4.6 Performance of the covering policy $\mathbf{v}^{*}$ in Algorithm 2 with respect to both cost criteria: maximum power and the total convex cost of power consumption, where malleability constraint pairs are i.i.d random vectors in $\Omega_{d}$, with $d=20$ and $n \in\{500,1000,1500, \cdots, 10000\}$. . . .

4.7 Performance of the covering policy $\mathbf{v}^{*}$ in Algorithm 2 with respect to both cost criteria: maximum power and the total convex cost of power consumption, where malleability constraint pairs are i.i.d random vectors in $\Omega_{d}$, with $d=100$ and $n \in\{500,1000,1500, \cdots, 10000\}$. . . .

4.8 Performance of the covering policy $\mathbf{v}^{*}$ in Algorithm 2 with respect to both cost criteria: maximum power and the total convex cost of power consumption, where malleability constraint pairs are i.i.d random vectors in $\Omega_{d}$, with $d=3$ and $n \in\{500,5500,10500, \cdots, 100000\}$. . .

4.9 Performance of the covering policy $\mathbf{v}^{*}$ in Algorithm 2 with respect to both cost criteria: maximum power and the total convex cost of power consumption, where malleability constraint pairs are i.i.d random vectors in $\Omega_{d}$, with $d=9$ and $n \in\{500,5500,10500, \cdots, 100000\}$. . . . 
4.10 Performance of the covering policy $\mathbf{v}^{*}$ in Algorithm 2 with respect to both cost criteria: maximum power and the total convex cost of power consumption, where malleability constraint pairs are i.i.d random vectors in $\Omega_{d}$, with $d=20$ and $n \in\{500,5500,10500, \cdots, 100000\}$. . .

4.11 Performance of the covering policy $\mathbf{v}^{*}$ in Algorithm 2 with respect to both cost criteria: maximum power and the total convex cost of power consumption, where malleability constraint pairs are i.i.d random vectors in $\Omega_{d}$, with $d=100$ and $n \in\{500,5500,10500, \cdots, 100000\}$. . .

4.12 Performance of the covering policy $\mathbf{v}^{*}$ in Algorithm 3 with respect to both cost criteria: maximum power and the total convex cost of power consumption, where malleability constraint pair is fixed and is in the

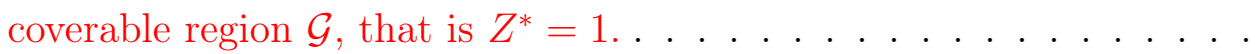

4.13 Performance of the covering policy $\mathbf{v}^{*}$ in Algorithm 4 with respect to Peak criteria, where malleability constraint pair is fixed, but is not in the coverable region $\mathcal{G}$, that is $Z^{*}<1 \ldots \ldots \ldots$. . . . . . .

4.14 Applying the covering policy for scheduling demands in time interval $[0, T]$. Demands are arriving in different time intervals of unit length and have be to be scheduled in the next time interval. The dotted horizontal line shows the average power consumption. . . . . . . . .

4.15 Long-Term performance of the covering policy $\mathbf{v}^{*}$ with respect to total operational convex cost. Malleability constraint pairs are i.i.d uniform random vectors in $\Omega_{d}$. The whole scheduling time interval is $[0,8]$. . .

4.16 Long-Term performance of the covering policy $\mathbf{v}^{*}$ with respect to Peak criteria. Malleability constraint pairs are i.i.d uniform random vectors in $\Omega_{d}$. The whole scheduling time interval is $[0,8] \ldots$. . . . . . . 71

5.1 The overall view of the proposed charging station . . . . . . . . 75

5.2 The out-flow of the state $g, i, j$ in the charging station . . . . . . . 78

$5.3 \gamma$ in optimal policy $z^{*}$ obtained from dynamic programming equations, where $(o)$ and $(*)$ correspond to $\gamma=0$ and $\gamma=1$ respectively . . . . 91

$5.4 \alpha$ in optimal policy $z^{*}$ obtained from dynamic programming equations, where $(o)$ and $(*)$ correspond to $\alpha=0$ and $\alpha=1$ respectively . . . .

$5.5 i_{R}$ in optimal policy $z^{*}$ obtained from dynamic programming equations, where $(o)$ and $(*)$ correspond to $(i-S)^{+}$and $\min (i, j)$ respectively . 
5.6 The Discounted Utility $\mathbf{U}(z)=-\mathbf{J}(z)$ per unit time for optimal policy is compared to heuristic policies "Grid-First" and "Storage-First" for different values of $\lambda$, where $V_{X}$ is constant. . . . . . . . . .

5.7 The Discounted Utility $\mathbf{U}(z)=-\mathbf{J}(z)$ per unit time for optimal policy is compared to heuristic policies "Grid-First" and "Storage-First" for different values of $\lambda$, where $V_{X}$ is variable. . . . . . . . . .

5.8 The average Utility $\mathbf{U}(z)=-\mathbf{J}(z)$ per unit time for optimal policy is compared to heuristic policies "Grid-First" and "Storage-First" for different values of $\lambda$, where $V_{X}$ is constant. . . . . . . . .

5.9 The average Utility $\mathbf{U}(z)=-\mathbf{J}(z)$ per unit time for optimal policy is compared to heuristic policies "Grid-First" and "Storage-First" for different values of $\lambda$, where $V_{X}$ is variable. . . . . . . . . . .

6.1 The out-flow of the state $X=\left(g, i^{1}, j^{1}, \ldots, i^{C}, j^{C}\right)$ in the charging station serving different classes of EVs . . . . . . . . . . . . .

6.2 The average Utility $\mathbf{U}(z)=-\mathbf{J}(z)$ per unit time for Real-Time and Off-line splitting of Power from the Grid optimal policies are compared to heuristic policies "Grid-First" and "Storage-First" for different values of $\lambda$, where $\mu^{1}=1$ and $\mu^{2}=2$. . . . . . . . . . . . . . . . 106

6.3 The average Utility $\mathbf{U}(z)=-\mathbf{J}(z)$ per unit time for Real-Time and Off-line splitting of Power from the Grid optimal policies are compared to heuristic policies "Grid-First" and "Storage-First" for different values

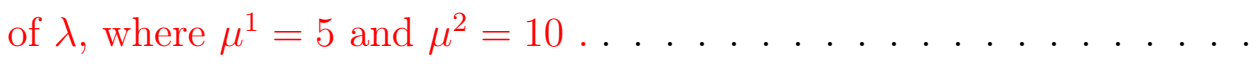

6.4 The average Utility $\mathbf{U}(z)=-\mathbf{J}(z)$ per unit time for Real-Time and Off-line splitting of Power from the Grid optimal policies are compared to heuristic policies "Grid-First" and "Storage-First" for different values of $\lambda$, where $\mu^{1}=[1,2,3,4,5]$ and $\mu^{2}=2 \mu^{1} \ldots \ldots \ldots$ 


\title{
Nomenclature
}

\author{
DSM Demand Side Management \\ EV Electrical Vehicles \\ FSP Fractional Strip Packing \\ PEV Plug-in Electrical Vehicle \\ PHEV Plug-in Hybrid Electrical Vehicle \\ MDP Markov Decision Process \\ PSP Power Strip Packing \\ RTP Real-Time Pricing \\ TOU Time of Use \\ TSP Traditional Strip Packing
}




\section{Chapter 1}

\section{Introduction}

Smart grid [1] is introduced to improve the overall performance of the existing electrical grid. It uses embedded measuring and controlling systems along with "Information Technology", to obtain the real time information about the state of the whole system and its numerous components. It uses this information to control the whole power grid by sending a variety of commands to some of the available facilities and services. Using these monitoring and control facilities, smart grid continually minimizes its operational costs and enhances the overall utility of the grid. Besides, it detects and diagnoses possible anomalies. It mitigates the effects of possible failures by avoiding them from spreading over the system and providing some tools for fast recovery.

Smart grid is expected to be a network of distributed and interconnected smart "MicroGrids" [2]. MicroGrids are clusters of electrical loads and local Distributed Generators (DG). They serve a variety of local electrical loads mainly by using their own DGs. DGs are micro-sources that mainly use renewable and intermittent energy resources like wind and sun. Each of these smart MicroGrids performs pervasive monitoring and control over its network (loads and sources). Furthermore, they incorporate a communication infrastructure to exchange data and commands with each other and the grid, in order to reliably meet their demands and optimize the utility of themselves and the whole electrical grid. These MicroGrids can operate whether they are connected to or isolated from each other and/or the electrical grid, which results in improving the reliability and Quality of Service by avoiding massive blackouts.

The classical electrical grid is, however, "Top-Down" oriented. There are some large power "generation" plants (power utilities) feeding into the power grid. These 
power plants are usually located far from urban areas. They transmit their produced electrical power via "transmission" lines toward the demand side and finally deliver the electrical power to their consumers via "distribution" system. The power utility always tries to provide maximum reliability by delivering requested demands by their customers. However, the electrical grid is "unidirectional", that is the utility doesn't have "real-time" information from demand side. Therefore, in order to assure maximum reliability, it is "over-engineered" to meet the maximum anticipated peak demand throughout its network. So, there are some reserve power supplies, which are only being used during peak hours and are out of service for the rest of the times. The problem is that these peak demands are not so frequent and only happen for limited durations of time. For example, about $20 \%$ of generation capacity exists only to meet the peak demands, so this capacity only is being used $5 \%$ of the time [3]. It causes the considerable waste of assets and capitals. Besides, these supplementary power supplies are generally produced from expensive natural non-sustainable resources, i.e. fossil fuels. Using these kinds of resources results in increasing the $\mathrm{CO}_{2}$ emissions and hence, increasing the electrical grid's total carbon footprint [4], [5].

Currently, we are facing the steadily rising demand for electrical power. Moreover, the increase in the number of the Plug-in [Hybrid] Electric Vehicles (PEVs/PHEVs) in the market, will increase the demand for electrical power even more. For example, during charging hours of PHEVs, the average household load is expected to be doubled [6]. This rise in demand along with limited capacity of the existing electrical grid, both in generation and transmission systems, results in decreasing the Quality of Service. More importantly, it will reduce the reliability of the grid, because it increases the probability of power failures due to sudden surges in electrical demand. This could impose a significant cost to the economy. As an example, in US, it is estimated that outages cost more than $\$ 100$ billion annually [7].

\subsection{Research Motivations}

To address the main issues of the classical grid, smart grid was introduced. However, with respect to value, size and geographical diversity of the existing electrical grid, it is not possible to drastically replace it with a smart grid. Instead, moving toward a smart grid is a gradual process starting by modernizing the current grid, optimizing its efficiency and finally making it more intelligent. Considering the fact that about $90 \%$ 


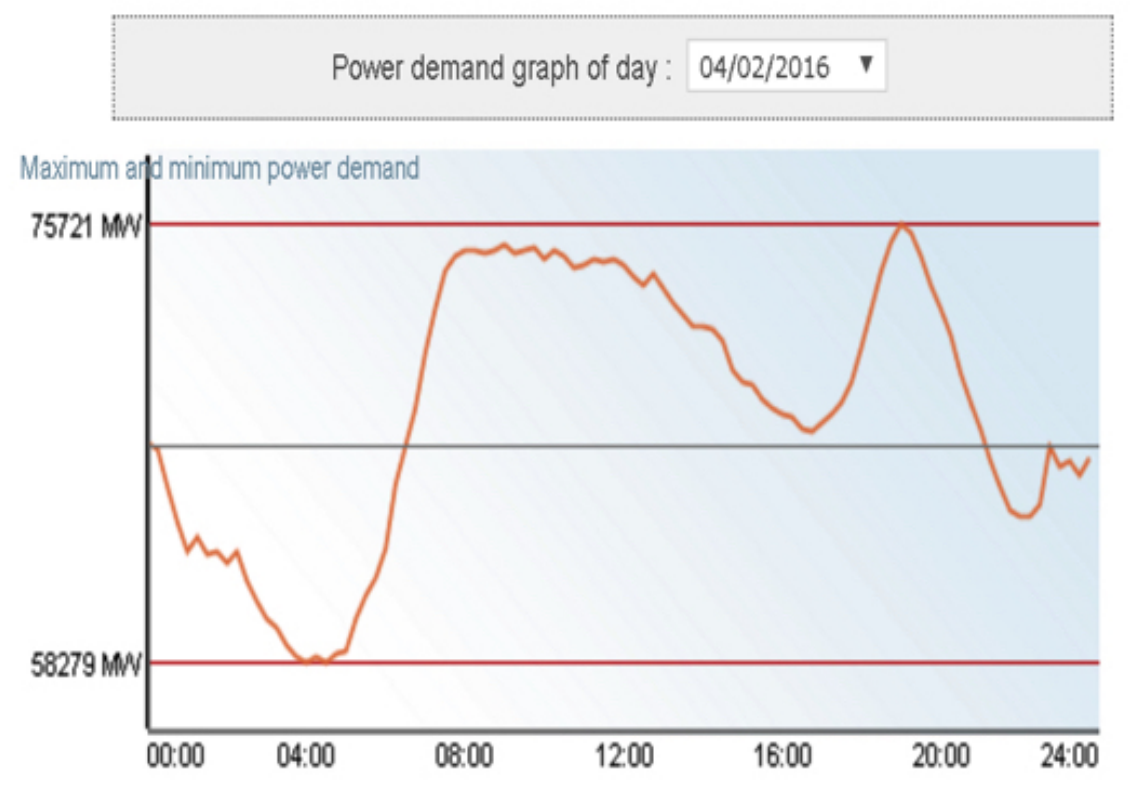

Figure 1.1: A typical daily load curve in an electrical grid. For example, this curve is computed based on the power consumption in France Power distribution network on February 4, 2016. [8].

of all disturbance and power outages are caused from issues in distribution system [3], it makes reasonable to start this process by changing and optimizing the consumption pattern in the distribution system. Indeed, without changing and improving the current "blind" consumption pattern on the demand side, we cannot stop wasting the assets and resources in the electrical grid. A consumption profile is the aggregation of individual end users' load profiles. For example, Figures 1.1 [8] and 1.2 [9] illustrate a typical daily load profile in an electrical grid. As we can see in these figures, during peak hours (e.g. 6 - 8 p.m. in Figure 1.1), the consumption rate is much higher than that of during off-peak hours (e.g. 3 - 6 a.m. in Figure 1.1). Then, as it is shown in Figure 1.2, it imposes the power utility to provide some reserve power supply to meet the demands during peak times and as we mentioned earlier it causes a considerable waste of assets in an electrical grid. On the other hand, if we can shape this load profile and make it more "even", we will be able to better utilize these supplementary power supplies and hence improve the efficiency of the assets in electrical grid.

In order to shape the load profile of the grid, the utility needs an infrastructure to exchange information and commands with its different components. So, it will be 


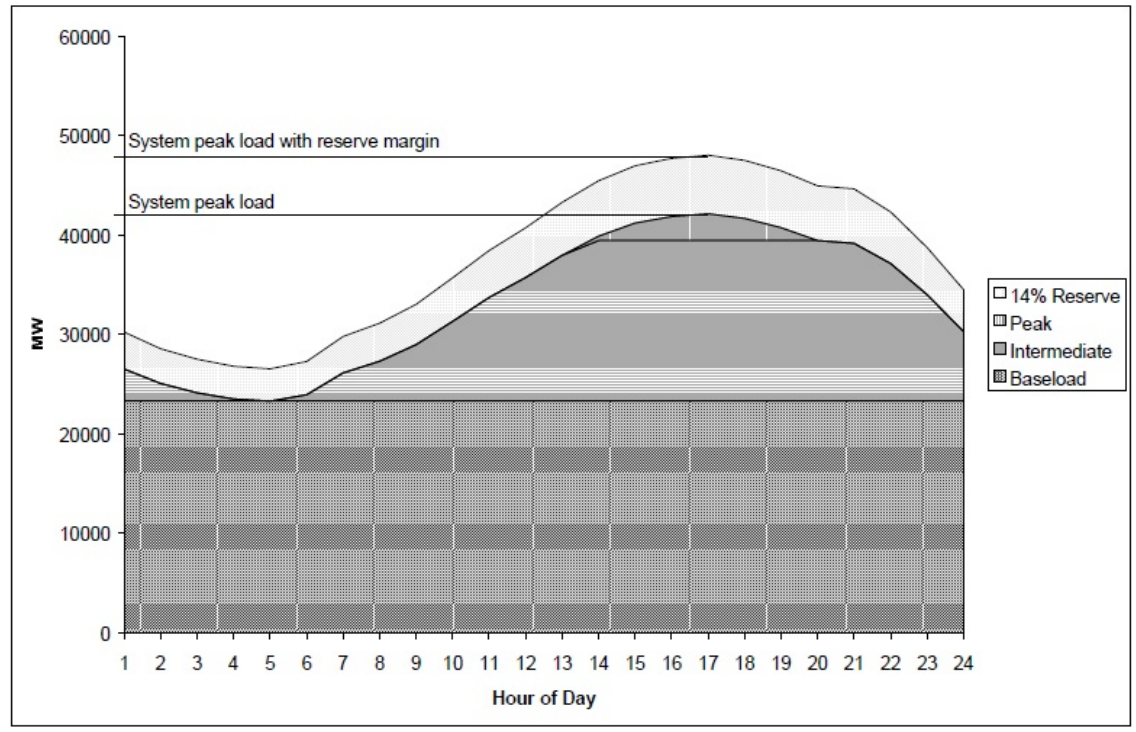

Figure 1.2: Electric Reliability Council of Texas (ERCOT) system load profile on August 27, 1990. This figure illustrates a typical commitment of generation assets. The reserve power supply is about 5.9 GW during peak hours [9].

able to perform more thorough and effective monitoring and control on the demand side. Advanced Metering Infrastructure (AMI) [10] was introduced to the electrical market to meet this requirement. It measures, analyzes and stores the energy usage data of electrical demands. Moreover, it sends necessary information to the utility operator and receives and performs a wide variety of commands from controller units. As a result, utilities are able to obtain the information from all over the grid and then by using this information, they will be able to devise and implement optimal control strategies and policies at demand side to enhance overall efficiency and utility of the grid.

Generally, the set of all efforts at the consumption side aiming at enhancing the efficiency and utilization of the power grid, is called "Demand Side Management (DSM)" [11]. It ranges from changing old and inefficient facilities to devising and deploying complex and sophisticated load management strategies. Demand Side Management does not necessarily decrease the total energy consumption. Instead, it mainly focuses on "shifting" some consumption demands from peak times to "offpeak" times. This load shifting will flatten the consumption profile of the system as much as possible. It then decreases the need for extra supplementary power supplies during peak hours and consequently enhances the utilization of the total power grid. Dynamic Pricing is one of the most popular tools being used in this respect [12,13]. 
As an example, in Time of Use (TOU) schemes [14], utility set different tariffs for power consumption in different times of the day.

However, due to limited flexibility of consumers and also with respect to actual requirements, users don't respond to energy prices that much and show only a minor shift in their consumptions behaviour [15]. Therefore, to optimize the utilization and minimize the operational cost of the grid, we need to perform more complex and sophisticated control schemes than just working with prices. This can be done by designing and deploying smart scheduling policies for serving the demands on the consumers' side.

\subsection{Research Overview and Contributions}

\subsubsection{Demand Side Management}

As we discussed earlier, one of the first and at the same time, most important steps toward enhancing the efficiency of the grid is changing the consumption pattern by deploying appropriate scheduling policies on the demand side of the grid. In designing such policies, we should take into account different natural and logistical constraints and limitations of different elements in the grid. It should be considered that most of these characteristics and constraints are "stochastic" in nature. Considering these parameters, will enable us to devise proper scheduling policies satisfying our optimization objectives. Our main contributions in this regard are as follows:

- In Chapters 3 and 4, we consider the problem of supplying electricity to a set of (large number of) customers in a smart-grid framework. Then we propose a new model for the demand side of the grid, as a set of "Malleable Rectangular-Shape" energy requirements. It means that each energy requirement has to be supplied without interruption in its service duration with a constant power intensity. In addition, its service duration is bounded by left- and right-malleability constraints, which are given system parameters. This model is motivated by the existence of electric appliances with flexibility on the charging rate. This model itself is novel in the smart grid literature. We will relate this model to the Strip Packing problem [16] with some modifications.

- In Chapter 3, we address the problem of scheduling electrical demands with fixed malleability constraints, where malleability constraint pairs are the same 
for all demands. Having the objective of minimizing the power peak as the cost, we first identify the lower bound on the optimal value of the power peak for all possible malleable constraint pairs. Then, we will propose a linear time algorithm that is asymptotically optimal.

- In Chapter 4, we extend the problem of scheduling malleable electrical demands, by letting each demand have its own malleability constraints (as an opposite to the unique pair in Chapter 3). In particular, we consider an asymptotic setting with a large number of relatively small energy demands. Such a setting enables us to include a convex cost in the treatment. Therefore, the objective will be finding a scheduling policy minimizing the power peak and the operational convex cost of the system while satisfying all the demands. Using an asymptotic analysis of stochastic demands, we will introduce the proper tight lower bounds for both types of costs in the system. Then, we will propose an on-line scheduling algorithm for demands with stochastic energy demands and stochastic malleability constraints and show that the presented algorithm is asymptotically optimal and has fully linear running time.

\subsubsection{Optimal Charging Station Strategy}

Due to public concerns about greenhouse emissions and high gasoline prices, Plug-in [Hybrid] Electric Vehicles (PEVs/PHEVs) are becoming more and more attractive for both consumers and industries. It is expected that PEVs will take $62 \%$ US market share of new car sales by the year 2050 [17]. This interest in PEVs is mainly due to high gasoline prices and public concerns about greenhouse emissions. In addition, by introducing Vehicle to Grid (V2G), it has been shown that in parking times, the electrical energy stored in PEVs, can be used as distributed energy resources to serve the rest of the electrical grid and alleviate the peak load on the grid $[18,19]$. Besides, the stored energy in PEVs can be used to enhance the reliability of micro grid networks, especially the ones using intermittent energy resources such as wind turbines, resulting in improving the QoS and stability of the micro grid network $[20,21]$.

In practice, the introduction of $\mathrm{PEVs}$ remains challenging. In particular, with respect to the size of the batteries deployed in PEVs, charging relatively large number of PEVs at the same time will impose a big stress on both the power generation 
and the distribution system [22]. For example, in [23] and [24], it is shown that simultaneous charging of only $5 \%$ of all PEVs will require additional $5 G W$ of electrical power by 2018 in the VACAR (Virginia - North Carolina- South Carolina) region. It will decrease the efficiency of the grid, and instead increase the need for extra supplementary power supplies during peak hours, which are mainly produced from non-green resources, such as fossil fuels. This contradicts one of the main purposes of using PEVs, i.e. decreasing the amount of greenhouse emissions by reducing the consumption of fossil fuels. To decrease the peak demand, different "Demand Side Management (DSM)" [11] techniques are deployed. Without necessarily decreasing the total energy consumption, DSM "shifts" some consumption demands from peak times to "off-peak" times to flatten the consumption profile of the system as much as possible and consequently enhances the utilization of the total power grid. DSM uses different schemes to influence the consumption pattern. Among them are some pricing schemes to encourage consumers to change their consumption behaviour in order to enhance the utility of the whole electrical grid. Time of Use (TOU) scheme is one of the most popular of such pricing schemes. In TOU scheme, different tariffs for the electrical power unit are set in different times of a day. For example, in Double Tariff scheme, which is very popular in the electricity market, consumers pay more per power unit during day-time in weekdays than during night-times and weekends.

In order to have better connection between the electricity prices and real-time situation of the grid, "Real-Time Pricing" (RTP) scheme has been introduced. In RTP scheme, the price of an electrical power unit is continuously updated with respect to the state of the grid at each time instance and sent to the consumers in real-time practically without delay [25]. Using RTP scheme, a utility can set the prices based on the state of the power generation, consumption rate and grid congestion at each time instance.

In some researches, the problem of charging PEVs and their impact on the electrical grid is studied by considering only residential (mostly night-time) charging. In [26] and [27], dynamic pricing being used as incentives to encourage the EV users to schedule charging and discharging their PEVs in order to reduce their cost and at the same time flatten the load curve of the grid. In [28], both centralized and decentralized models are used to mitigate the impact of residential charging on peak load of the grid. Unfortunately, due to limited flexibility of consumers, they don't respond to energy prices as much and show only a minor shift in their consumption 
patterns [15]. Therefore, in addition to offering these types of incentives, we need to develop more complex and sophisticated control policies resulting in better overall performance of the electrical grid.

Relying only on residential charging cannot even fully address the charging requirements of PEVs (See Chapter 2, Section 2.3). For example, by deploying only night-time charging, an average size PEV needs a battery with a capacity of 40 miles per full charge; this necessitates the deployment of relatively big and expensive batteries for PEVs. Using charging stations decreases this value to 13 miles per full charge [29]. It then decreases the size of the battery and consequently reduces PEV prices in the market and makes it more competitive with internal combustion engine vehicles. It has been shown that ease of recharging PEVs is a major factor for consumers for buying electric cars [30]. So, deploying public PEV charging stations facilitates the use of PEVs and consequently enhances their place in the market. The same as gas stations, these charging stations should be easily accessible for consumers in both urban and non-urban areas. However, due to longer charging process of PEVs, gas stations cannot be used for both PEVs and internal combustion engine vehicles at the same time [31]. Therefore, we have to design and deploy PEV specific charging facilities, which could satisfy the special requirements of both PEVs and the modern smart grid. In particular, a charging station should deploy a suitable scheduling policy for serving PEVs. Especially, these policies should take into account different objectives such as: the overall efficiency and reliability of the grid, users' satisfaction (QoS), and eventually the long term profit of deploying the charging station.

As part of this thesis, we will consider the problem of finding an optimal control policy for a charging station of PEVs. Our main contributions are as follows:

- In Chapter 5, we address the problem of serving PEVs in a charging station using a local storage energy unit with finite capacity. In particular, we take into account the price fluctuations for electrical power by considering a Real-Time Pricing scheme. Our goal is to find a control policy minimizing the operational cost of the charging station, while considering the stability of the grid and Quality of Service. So, we first model the charging station as a continuous time Markov Decision Process with three control actions at each state: whether to accept or block the arriving demands, determining the charging rate of the local storage unit and deciding on the number of demands being served by the storage unit. This model enables us to use some well-known mathematical techniques in 
this field, especially Dynamic Programming Techniques, which are widely used in Network Optimization problems. Then we prove the existence of a stationary and bang-bang optimal policy minimizing a discounted cost over an infinite time horizon. Eventually, we extend our results and determine the optimal policy minimizing the infinite horizon average cost.

- In Chapter 6, we extend the problem introduced in Chapter 5, by letting the arriving PEVs belong to different classes of batteries and hence have different charging rates. Similar to the single-class scenario, the charging station is modeled as continuous-time Markov Decision Process. A controller of a charging station should again continuously decide about: whether to accept or block an arriving customer, how much of the energy demands should be met using the local storage units, and what should be the charging rate of each local storage unit. In addition to these control actions, the controller has to decide how to split the available power from the grid between different classes of PEVs. We will first derive the resulted optimization problem for the cases where splitting decision is made offline, i.e. before serving the PEVs starts. Then, we go further and present the formulation for the optimization problem for real-time splitting of the available power form the grid; that is splitting decisions are made continuously while serving the customers.

\subsubsection{Published and Submitted Papers}

Based on the work done in this thesis, the following papers have been published or submitted:

- Journal papers:

- M. M. Karbasioun, I. Lambadaris, G. Shaikhet, and E. Kranakis, "Optimal Controlling of Charging Stations for Heterogeneous Electric Vehicles", Submitted to the journal of Computer Communications, 2018 [32].

- M. M. Karbasioun, G. Shaikhet, I. Lambadaris, and E. Kranakis, "Asymptotically Optimal Scheduling of Random Malleable Demands in Smart Grid," The journal on Discrete Mathematics, Algorithms and Applications (DMAA), vol. 10, no. 2, 2018 [33].

- Conference Papers: 
- M. M. Karbasioun, I. Lambadaris, G. Shaikhet, and E. Kranakis, "Optimal charging strategies for electrical vehicles under real time pricing," in IEEE International Conference on Smart Grid Communications (SmartGridComm), pp. 746-751, IEEE, 2014 [34].

- M. Karbasioun, G. Shaikhet, E. Kranakis, and I. Lambadaris, "Power strip packing of malleable demands in smart grid," in IEEE International Conference on Communications (ICC), Budapest, Hungary, 2013 [35].

- G. Shaikhet, M. Karbasioun, E. Kranakis, and I. Lambadaris, "Asymptotic convex optimization for packing random malleable demands in smart grid," in IEEE International Conference on Communications (ICC), Budapest, Hungary, 2013 [36].

\subsection{Thesis Organization}

The rest of the thesis is organized as follows:

- In Chapter 2, a literature review as well as research background is presented. Especially, we will review some major studies in Demand Side Management, Strip Packing Problem and Charging Station Control.

- In Chapter 3, we are introducing the problem of supplying electricity to "Rectangular-Shape" demands with stochastic energy requirements and fixed malleability constraint pair. Then, we will introduce a linear time algorithm that minimizes the power peak and is asymptotically optimal.

- In Chapter 4, we consider an asymptotic setting with a large number of relatively small energy demands. In the new introduced setting, each demand will have its own stochastic scheduling constraints. Furthermore, we will include a convex cost as the cost criteria for evaluating the performance of the grid operator. We will finish the chapter by introducing an asymptotically optimal algorithm with respect to both types of costs, i.e. power peak and the total convex cost.

- In Chapter 5, we will study the problem of optimally controlling a charging station of PHEVs in which PHEVs are charged either directly from the electrical grid or from a local storage unit. First, we model the charging station as a 
continuous time Markov Decision Process. Then after introducing a proper cost function, we will show how to find the optimal policy.

- In Chapter 6, we extend the problem introduced in Chapter 5, by letting the arriving PEVs belong to different classes of batteries and hence have different charging rates. In this case, the charging station will deploy a local storage unit for each class of customers. We start our analyses by presenting the resulted mathematical model, then we will show how to find the corresponding optimal policies for controlling the charging station. In particular, in addition to all control actions mentioned in Chapter 5, the controller has to decide how to split the available power from the grid between different classes of customers. We find optimal policies in two distinct scenarios: In the first scenario, the splitting decision is made off-line; that is before serving the customer starts. In the second scenario, we formulate and solve the optimization problem for RealTime splitting of the available power from the grid between different classes of PHEVs, i.e. while serving the demands.

- In Chapter 7, we will summarize the works discussed in this thesis and highlight the next steps for future works. 


\section{Chapter 2}

\section{Background and Related Works}

Wisely designing and implementing a scheduling policy plays a crucial role in Demand Side Management (DSM) [7]. The main goal of DSM is enhancing the efficiency and Quality of Services in the grid network while reducing the total cost of using the (limited) resources of the grid. This is usually done by smart exploitation of some statistical characteristics of (stochastic) demands and then shaping the load profile of the system as much as the natural or logistical constraints permit. Scheduling policies use different schemes to influence the consumption pattern, and consequently minimize the operating cost of the grid. In Section 2.1, we will review these efforts.

Different techniques are being used to model the demand side of the smart grid. In Chapters 3-4, we modelled electrical energy requirements as "rectangular" shape demands, meaning that each demand has to be supplied without interruption with a constant power intensity during the time been served. The resulted setting resembles a so-called Strip Packing Problem. In Section 2.2, after introducing Strip Packing Problem, we will review major works in this area. Then we will consider some efforts in smart grid literature using Strip Packing in their models.

Eventually with the expected presence of Plug-in [Hybrid] Electric Vehicles (PEVs/PHEVs) to the market, designing and deploying suitable charging stations with proper scheduling schemes is a vital part in Demand Side Management (Especially due to the fact that charging a large number of PHEVs at the same time, will push the peak demand in the electrical grid [6]). Several efforts aimed at designing

and deploying efficient charging stations, will be reviewed in Section 2.3, which are the most related works to our study in Chapter 5 . 


\subsection{Demand Side Management}

Generally, the set of all efforts at the consumption side aiming at enhancing the efficiency and utilization of the power grid, is called "Demand Side Management (DSM)" [11]. It ranges from changing old and inefficient facilities to devising and deploying complex and sophisticated load management strategies. Demand Side management does not necessarily decrease the total energy consumption. Instead it mainly focuses on "shifting" some consumption demands from peak times to "offpeak" times $[13,37]$. This load shifting will flatten the consumption profile of the system as much as possible. It then decreases the need for extra supplementary power supplies during peak hours and consequently enhances the utilization of the whole power grid.

Demand side management deploys different schemes to influence consumption pattern. For example, in Direct Load Control (DLC) [38] schemes, the utility can directly control the energy consumption rate of some loads on the customer side. On the other hand, in Demand Response (DR) [39], utilities don't directly control the demands. Instead the utility sends some signals and information an incentives to the users to encourage them to shift and reschedule their demands and/or change their consumption patterns. Dynamic Pricing is one of the most popular schemes being used in this respect $[12,13,27]$. As an example, in Time of Use (TOU) schemes [14], utility set different tariffs for power consumption in different times of the day. Among TOU schemes, Double Tariff is very popular in electrical market, where consumers pay more per power unit during day-time in weekdays than during night-times and weekends. In most of TOU schemes, the rates of electrical power for different times of the day are usually assumed to be set at the beginning of the season and remain fixed during that season. However, in some TOU schemes, the prices are fluctuating more frequently, and the prices of using electricity in different times in a day are sent to the users at least a day ahead. In order to have better connection between the electricity prices and real-time situation of the grid, "Real-Time Pricing" (RTP) scheme has been introduced [25]. In RTP scheme, the price of an electrical power unit is continuously updated with respect to the state of the grid at each time instance and sent to the consumers in real time practically without delay. In RTP the granularity of prices, i.e. the time where retail electricity prices remain unchanged, is about an hour in most cases, which is much smaller than that of in TOU scheme, which is about 8 
hour [25]. However due to limited flexibility of consumers and also with respect to physical world requirements, users don't respond to energy prices that much and show only a minor shift in their consumptions behaviour [15]. Therefore, to optimize the utilization and minimize the operational cost of the grid, we need to perform more complex and sophisticated control schemes than just working with prices.

Almost in all DSM schemes, the main goal is minimizing the total cost of using the (limited) resources of that network and hence enhancing the efficiency of the grid $[12,27,40,41]$. While in many studies, such as [12,27,37,41], the Peak to Average Ratio (PAR) of the energy consumption rate is considered as the cost criteria for the grid, others are also taking into account the total convex cost of using the grid resources [40,42-44]. Even though these two costs are somehow related to each other, they are not the same. The cost of consuming the energy is considered to be a convex function of instantaneous total power consumption due to the fact that each additional unit of power needed to serve demands is more expensive as the total power demand increases [44]. This is because the supplementary power for serving the demands when the consumption rate is high, is generally produced from expensive natural non-sustainable resources e.g. fossil fuels.

To achieve the goal of minimizing the cost of the grid, researchers are deploying different techniques and approaches. In [44-47] centralized control policies are deployed to minimize the cost of the grid, where $[26,40,42,43,48]$ chose distributed algorithm to achieve the same goal. In [42] and [43], demands are assumed to be deterministic and known in advance for controllers, while many studies consider stochastic demands and/or suppliers in their setting [20,21,44,45]. For example, in [44] and [45], some queueing analyses alongside Dynamic Programming techniques are being used to devise on-line scheduling schemes for the random arriving demands with flexibility of delaying the start of their service. Specially in [44], by using a threshold policy, they proposed an asymptotic scheduling policy to minimize the total convex cost of the consumed power in the system. In their setting the running time of the system is assumed to be infinite as well as the acceptable delay for starting the serving of each demand. In [49], it is assumed that demands have different stochastic power requirements and durations and then authors try to propose an on-line energy storage control policy that minimizes long-term average convex operational cost of the grid, in the presence of a storage unit. Using game theory, in [40,42,43] authors propose a distributed algorithm for serving the deterministic demands. In [40], it was 
assumed that multiple energy suppliers being available and then they considered two non-cooperative games, the one on supplier side and another one on the demand side. In [42] and [43], assuming a central energy supplier, the game on customer side was studied. Especially in their model, the power network consists of demands each with its own energy demand and with its own minimum and maximum acceptable power level that should be scheduled in their own requested time intervals. The primary goal of their algorithm is reducing the total convex cost of the network, while they still show that their algorithm can be used to reduce the PAR in the network. The problem in [42] is off-line Scheduling of deterministic demands, since complete knowledge of the demands, such as number of demands and the amount of energy needed by each of them are known in advance. Other scenarios can happen when the complete knowledge of the demands is not available and we may need to perform on-line scheduling. For example, in [46] authors try to find algorithms for different levels of knowledge about the demands such as arrival times, durations and power intensities. Again, the goal is reducing the total convex cost of the power consumption in the system.

In the sequel, in Chapter 3, considering Peak to Average Ratio (PAR) cost criteria, we will pursue the goal of minimizing the maximum total consumption rate in a grid. Then in Chapter 4, in addition to PAR cost criteria, we will include the total convex cost of the grid in our analyses. In general, in both Chapters 3 and 4, we will propose some centralized scheduling policies, which flatten the consumption rate of the grid, as much as possible, and consequently minimize the total cost of the grid. Eventually in Chapter 5, after introducing a model for a charging station of PEVs, we are proposing a control policy minimizing the operational cost of the charging station. In particular, we assume that the price of the electrical power unit is determined by a Real-Time Pricing scheme, where the price of the electrical power is continuously being updated with respect to the state of the grid at each time instance. Furthermore, we will isolate the fluctuations in the total energy consumption rate of the proposed charging station from the grid, by imposing the assumption that the electrical power drawn from the grid by the charging station is bounded. Eventually, we will employ a local energy storage unit, to serve PHEVs in hi-demand time periods, and get charged in low-demand ones. 


\section{2 scheduling of random malleable demands}

To design efficient scheduling policies for an electrical grid, we first need to have an appropriate model to represent the grid. In this model, we should take into account physical and statistical characteristics as well as inherent and logistical constraints and limitations of different components of the electrical grid. Most of these characteristics and constraints are "stochastic" in nature. Taking advantage of such statistical characteristics, we will be able to come up with satisfactory models and then develop appropriate scheduling policies to control and modify the load profile of the system, with respect to our desires.

In Chapters 3 and 4, we consider a problem of supplying electricity to a set of (large number of) customers in a smart-grid framework. In these Chapters, we model the demand side of the grid, as a set of "Malleable Rectangular-Shape" energy requirements. By "rectangular-shape" demands, we mean each energy requirement $A_{i}$, in $\mathcal{N}=\left\{A_{i}, i=1, \ldots, n\right\} \in \mathbb{R}_{+}^{n}$, requires a certain amount of electrical energy and has to be supplied without interruption in some interval $\left[\tau_{i}, \tau_{i}+s_{i}\right]$ with a constant power intensity $d_{i}=\frac{A_{i}}{s_{i}}$, assuming $0 \leq \tau_{i} \leq \tau_{i}+s_{i} \leq 1, \quad i=1, \ldots, n$. In addition, each demand is "Malleable"; that is, the energy requirement $A_{i}$, has to be served with possible service time duration $s_{i}$, between the malleability constraint pair $\ell_{i}$ and $r_{i}$, which are given system parameters $\left(\ell_{i} \leq r_{i}\right)$ (Figure 2.1). In particular, a controller has to determine a scheduling policy $\pi=\left\{\left(\tau_{i}, s_{i}\right), i=1, . ., n\right\}$, so that for each $i$ :

- The demand $A_{i}$ is being supplied on $\left[\tau_{i}, \tau_{i}+s_{i}\right] \subset[0,1]$, with a constant power $A_{i} / s_{i}$,

- Its duration $s_{i}$ satisfies: $\ell_{i} \leq s_{i} \leq r_{i}, \quad i=1, \ldots, n$.

Then at each moment of time, the total energy consumption rate (i.e, total power $P(t)$ ) of the grid is the sum of all consumption rates of the demands being supplied at that moment.

\subsubsection{Strip Packingproblem and the related literature}

Our setting resembles a so-called strip-packing problem [16,50,51]. Indeed, by viewing the demands as rectangles, we want to pack them with their side $s_{i}$ parallel to 


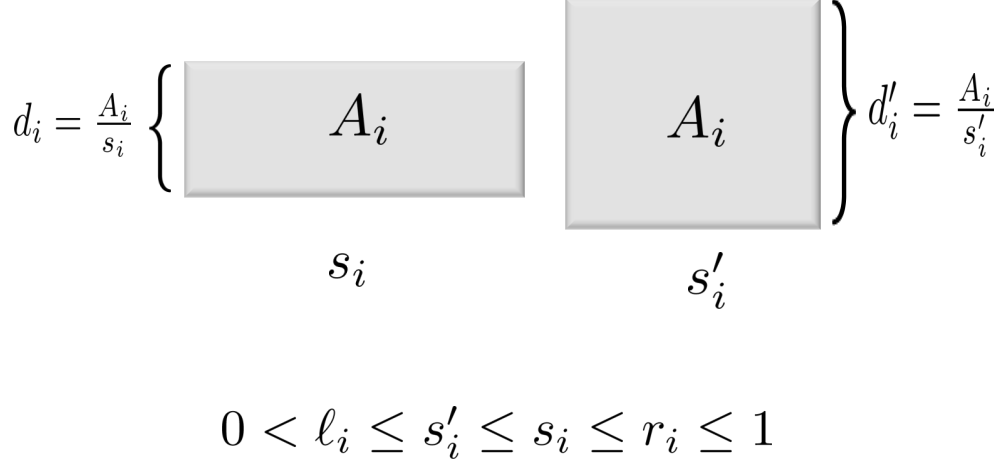

Figure 2.1: Malleable rectangular Shape Energy Demand representation.

horizontal axis in a rectangular bin of width $[0,1] \times P_{\text {opt }}$ where an optimal height $P_{\text {opt }}$ is unknown. The problem is known to be NP complete (see [52]) and therefore an optimal height cannot always be computed in polynomial time.

Strip packing problem (also called two-dimensional strip packing) is a variant of the bin-packing problem $[53,54]$ in which rectangles are packed into a strip of width 1 and infinite height in a way that rectangles don't overlap each other [52]. Strip packing has been extensively explored in the literature, where some heuristics [16, 51,55-60], as well as polynomial approximations [50,61,62] for Strip Packing problem were proposed. ( [52] and [63] are presenting a good survey of some of the important works in this area). The objective in many works related to Strip Packing is minimizing the maximum height of the packing in the strip, i.e. minimizing the $H$, where $H=H_{\text {max }}=\max _{t \in[0,1]}\{H(t)\}$. More precisely, for a given set of demands, $\mathcal{N}$, let $H_{\text {opt }}(\mathcal{N})$ denote the optimum solutions of Strip Packing problem and $\mathcal{H}(\mathcal{N})$ denote the output of the algorithm $\mathcal{H}$. The absolute performance ratio of $\mathcal{H}$, also known as worst case ratio, is defined as $\sup _{\mathcal{N}} \mathcal{H}(\mathcal{N}) / H_{\text {opt }}(\mathcal{N})$ and the asymptotic performance ratio of $\mathcal{H}$ (also known as asymptotic worst-case ratio)is defined as $\lim _{H_{\text {opt }}(\mathcal{N}) \rightarrow \infty} \sup _{\mathcal{N}} \mathcal{H}(\mathcal{N}) / H_{\text {opt }}(\mathcal{N})$ (used for the cases where we are dealing with large numbers of demands). The value of the demands can be considered as random values with a certain distribution or as deterministic values. The performance of a scheduling policy in both cases can be measured by worst case analyses [64]. For 
stochastic demands, average case analyses [65] is also being used.

Scheduling algorithms in Strip Packing are mainly divided into two categories, online and off-line algorithms. An algorithm is called on-line, when demands arrive one by one and then a demand $A_{i}$ is scheduled without knowledge of next demands, i.e. $A_{i+1}, \cdots A_{n}$ [53], while off-line algorithms need the information of all the demands. For off-line scheduling problem, most commonly referred work is [50] which has an asymptotic worst case performance ratio $(1+\epsilon)$ while its running time is polynomial in both $n$ (the number of demands) and $(1 / \epsilon)$ (Algorithms with such running times, are known as "Fully" polynomial algorithms). On the other hand [66] proposes an online scheduling policy with the same performance ratio for on-line problem while the running time is just linear in the number of demands, $n$ and worse than exponential in $(1 / \epsilon)$.

The most related works in strip packing literature to our work are [66] and [67]. In [67], authors generalize the setting of [50] to find an off-line scheduling policy for Malleable Tasks where each task could use different number of resources (e.g. processor, memories, ...) which can also alternate their service times. The same as [50], the proposed algorithm is fully polynomial and has an asymptotic worst case performance ratio $(1+\epsilon)$. In [66], authors propose an on-line scheduling policy for malleable demands where demands can be lengthened while their areas remain fixed. In their setting the height of each rectangular demand is below bounded by its initial height but does not have upper bound for the heights of the rectangles. The values of demands (their width and heights) are unknown but deterministic. The main drawback of this algorithm is the running time in term of $(1 / \epsilon)$, which is worse than exponential.

The scheduling problem in this paper (which is called Power Strip Packing (PSP) in the sequel) is different from strip packing problem (we call it Traditional Strip Packing (TSP)) in some aspects. In TSP, as shown earlier, the height $H^{\pi}(t)$ at any time $t$, for a scheduling policy $\pi=\left\{\left(\tau_{i}, s_{i}\right), i=1, . ., n\right\}$, is defined as the uppermost boundary of scheduled rectangles at time $t$, while in PSP the height of the strip packing at time $t$ or equivalently the total electrical power, $P(t)$, of the grid is obtained from Equation (2.1), as follows:

$$
P^{\pi}(t)=\sum_{i=1}^{n}\left(\frac{A_{i}}{s_{i}} \cdot 1_{\left\{\tau_{i} \leq t \leq \tau_{i}+s_{i}\right\}}\right), \quad 0 \leq t \leq 1 .
$$




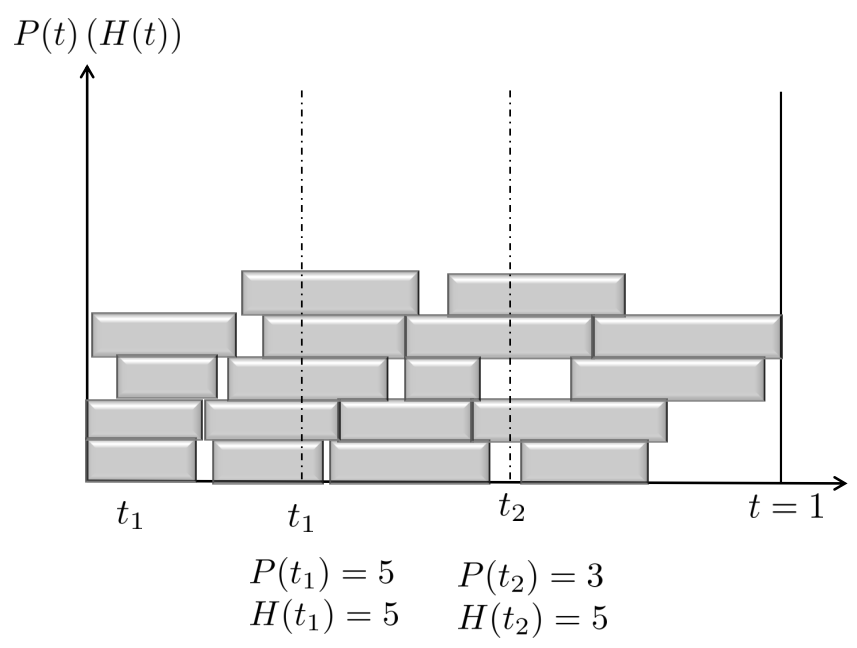

Figure 2.2: Different interpretations of power and height in PSP and TSP. For simplicity the height of each rectangle is assumed to be 1. In PSP: $P\left(t_{1}\right)=5$ and $P\left(t_{2}\right)=3$ where in TSP $H\left(t_{1}\right)=H\left(t_{2}\right)=5$.

and consequently,

$$
P_{\max }^{\pi}=\max _{t \in[0,1]}\left\{P^{\pi}(t)\right\},
$$

This difference arises from the nature of the electric power, in which the overall height (i.e. power) at any given time is just the sum of the scheduled (i.e. active) demands (See Figure 2.2). Naturally, $P^{\pi}(t) \leq H^{\pi}(t)$ for any policy $\pi$ and consequently $P_{\max }^{\pi} \leq H_{\max }^{\pi}$. Another difference which also arises from the nature of power demands, is that demands can overlap each other and then the amount of consumed power at each time is the sum of all scheduled demands at that time. In the next chapters, we will show that our algorithms and analyses are applicable for both Traditional Strip Packing and Power Strip Packing problems.

There are some studies in Smart Grid literature, in which similar models are being used for representing the energy requirements in the grid. For example, in [44] and [45], demands are considered as rectangles with given service durations, $s_{i}$, and power demands, $d_{i}$. Besides, they are assumed to be flexible with respect to delaying the start of their service. Then using a threshold policy, an asymptotic scheduling policy is proposed to minimize the total convex cost of the consumed power in the 
system. Especially in their final setting the running time of the system is assumed to be infinite as well as the acceptable delay for starting the serving of each demand. In [49], each demand is assumed to get served with a constant random power intensity for a random duration of time. Considering this model, an on-line energy storage control policy is devised, which minimizes long-term average convex operational cost of the grid, in the presence of a storage unit. In [46], demands are assumed as rectangles with given service times $s_{i}$ and power intensity $d_{i}$, but flexible start time $\tau_{i}$. Then after showing that in general finding the optimal solution is NP hard, they tried to find some approximations for optimal policy in the presence of different levels of knowledge about the demands such as durations and power intensities. Eventually in [68-71], a set of rectangular-shape electrical demands are considered, where for each demand the power intensity and service duration is known in advance. In addition, by having the flexibility of delaying the starting time of the service, it is assumed that all demands have the same earliest start time and deadline for receiving their energy requirement. In particular, authors considered two main situations, where demands are assumed to be preemptive and non-preemptive. Then with the objective of minimizing the peak power, they proposed scheduling policies using integer linear programming, as well as next-fit decreasing height heuristic [16] and then compared the results for preemptive and non-preemptive cases.

In Chapters 3, we will explore the problem of scheduling rectangular-shape electrical demands in a finite interval $[0,1]$, while considering fix malleability constraint pair $(\ell, r)$ for the time duration of serving each demands. In our setting we assume that the demands are non-preemptive, i.e. it has to be be supplied without interruption in their service interval. Eventually we will propose a linear time algorithm minimizing the power peak and show that this algorithm is asymptotically optimal. Then in Chapter 4, we will extend the Power Strip Packing problem by assuming each demand has its own malleable constraint pair, $\left(\ell_{i}, r_{i}\right)$. In particular, in this Chapter, we will consider an asymptotic setting with a large number of relatively small energy demands. Furthermore, we will include the total convex cost in our treatment. Therefore, the objective will be finding a scheduling policy minimizing the power peak and/or the operational convex cost of the system while satisfying all the demands. 


\subsection{Optimal Controlling of Charging Stations for Electrical Vehicles}

Plug-in [Hybrid] Electric Vehicles (PHEVs/PEVs) have attracted the interest of both industry and consumers. It is expected that PEVs will take $50 \%$ market share of new car sales by the year 2050 [72]. The estimate for US market is even higher and is expected to be $62 \%$ [17]. This interest in PEVs is mainly due to high gasoline prices and public concerns about greenhouse emissions. In addition, by introducing Vehicle to Grid (V2G), it has been shown that in parking times, the electrical energy stored in PEVs, can be used as distributed energy resources to serve the rest of the electrical grid and alleviate the peak load on the grid $[18,19]$. Besides, the stored energy in PEVs can be used to enhance the reliability of micro grids networks, especially the ones using intermittent energy resources such as wind turbines, resulting in improving the QoS and stability of the micro grid network [20,21].

In particular, with respect to the size of the batteries deployed in EVs, charging relatively large number of EVs at the same time will impose a big stress on both the power generation and the distribution system [22]. For example, in [23] and [24], it is shown that simultaneous charging of only $5 \%$ of all EVs will require additional $5 G W$ of electrical power by 2018 in the VACAR (Virginia - North Carolina- South Carolina) region. It will decrease the efficiency of the grid, and instead increase the need for extra supplementary power supplies during peak hours, which are mainly produced from non-green resources, such as fossil fuels. This contradicts one of the main purpose of using EVs, i.e. decreasing the amount of greenhouse emissions by reducing the consumption of fossil fuels. In some studies, it is tried to address this issue, considering only residential (mostly night-time) charging. In [26] and [27], dynamic pricing being used as incentives to encourage the PEV users, to schedule charging and discharging their PEVs in order to reduce their cost and at the same time flatten the load curve of the grid. Also in [28], both centralized and decentralized models are being used to mitigate the effect of residential charging on peak load of the grid.

However, only considering residential charging cannot solve the problem of charging PEVs. For example, by deploying only night-time charging, an average size PEV needs a battery with a capacity of 40 miles per full charge; this necessitates the deployment of relatively big and expensive batteries for PEVs. However, using charging 
stations will decrease this value to 13 miles per full charge [29], which will decrease the size of the battery and consequently reduce PEV prices in the market and make it more competitive with internal combustion engine vehicles. It has been shown that ease of recharging PEVs is a major factor for consumers for buying electric cars [30]. So, deploying public PEV charging stations will facilitate the use of PEVs and hence enhance their place in the market. The same as gas stations, these charging stations should be easily accessible for consumers in both urban and non-urban areas. However due to longer charging process of PEVs, gas stations cannot be used for both PEVs and internal combustion engine vehicles, at the same time [31]. Therefore, we have to design and deploy PEV specific charging facilities, which address PEVs and modern smart grid requirements. One of the main considerations in deploying a charging station is having a suitable scheduling policy for serving PEVs. Especially these policies should take into account different objectives, such as grid efficiency and reliability, user satisfaction and QoS, and eventually and essentially charging station profit.

In Chapters 5 and 6, we will address the problem of serving PEVs in a charging station. In particular, we model the charging station as a continuous time Markov Decision Process(MDP). Then we will propose a control policy minimizing the operational cost of the charging station, while considering the stability of the grid and Quality of Service. In the sequel, we first provide some technical background with respect to Markov Decision Processes and then will review some of the most relevant studies to our research, which is presented in Chapters 5 and 6.

\subsubsection{Markov Decision Processes}

\subsubsection{Definitions}

Markov decision processes(MDPs) are models for sequential decision-making when outcomes are uncertain. It helps us to model a decision making problem in cases where outcomes are partly random and partly under the control of a decision maker. The controls will be applied at transition instances so that an appropriate cost is optimized. (That is the reason, MDPs are sometimes called stochastic control processes.). The Markov decision process model mainly consists of states, actions, decision epochs, transition probabilities and costs. If transitions happen (and consequently, decisions 
are made) at discrete time intervals, the process is called discrete-time Markov Decision Process. However, in continuous-time Markov Decision Processes, transitions can happen (and decisions can be made) at any time. We denote, discrete-time Markov Decision Process by $\left\{X_{k}: \quad k \geq 0\right\}, k \in \mathbf{N}$ and continuous-time Markov Decision Processes by $\left\{X_{t}: t \geq 0\right\}, t \in \mathbf{R}^{+}$. In any case, the stochastic process takes its value either from a finite set (e.g. $\{1,2, \ldots, n\}$ ) or a infinite but countable set (e.g. $\{1,2,3, \ldots\} \equiv \mathbf{N}$ ), which is called the state space (We denote the state space by $\mathcal{X}$ ). In the sequel, unless stated otherwise, we assume that the Markov Process is discretetime. At any given time epoch $k$, when the system is in state $X$, the decision maker chooses an action $\mathbf{u}_{X, k}$ from a certain action set that may depend on the observed state ${ }^{1}$. We denote the action set in state $X$ by $\Omega_{X}$. Similar to the state space $X$, the action sets $\Omega_{X}$ may be finite, countable infinite, non-countable but compact or more general. The set of all decision rules $\mathbf{z}=\left\{\mathbf{u}_{X, k}: \mathbf{u}_{X, k} \in \Omega_{X}: \quad X \in \mathcal{X}, k \geq 0\right\}$ is called a control policy. For any given state of the system and the chosen action at any time epoch, an immediate cost is incurred by the system. In general, this cost may depend on the decision time epoch, the state of the process and the chosen action, but not on the history of the process. The controllers apply the actions at transition instances so that an appropriate cost is optimized over the control horizon, which is the entire time interval at which the system has to be controlled. This horizon may be finite, infinite or of a random length.

A Markov decision process is indeed an extension of Markov chains with the addition of actions and costs. The state transitions in a Markov chain (and a Markov Decision Process) satisfy the "Markov property". It means that at any time epoch $k \geq 0$, the state at the next time epoch depends on the decision time epoch $k$, the state of the system at that time epoch $X_{k}$ and the chosen action $\mathbf{u}_{x, k}$ and not on the history of the process. That is:

$$
\begin{aligned}
& P\left(X_{k+1}=X^{\prime} \mid X_{k}=X, \mathbf{u}_{X, k}=u, X_{k-1}=X^{\prime \prime}, \mathbf{u}_{X^{\prime \prime}, k-1}=u^{\prime \prime}, \ldots, X_{0}=X^{0}, \mathbf{u}_{X^{0}, 0}=u^{0}\right) \\
= & P\left(X_{k+1}=X^{\prime} \mid X_{k}=X, \mathbf{u}_{X, k}=u\right)
\end{aligned}
$$

Equation (2.3) states that the future state of the system only depends on its current state and the current action, and is conditionally independent of all previous states

\footnotetext{
${ }^{1}$ Note that in this study, we study the Markov Decision Process under the complete observation assumption, i.e. the decision maker observes that the system is in state $X$, iff the system is actually in state $X$.
} 
and actions. That is the reason Markov property is also called memoryless property. If the transitions also depend on the decision time epoch $k$, then the process is called non-stationary. Otherwise, it is stationary. Therefore, in a stationary Markov Process, all the actions are chosen according to a stationary policy $\mathbf{z}=\left\{\mathbf{u}_{X}: \mathbf{u}_{X} \in \Omega_{X}: X \in\right.$ $\mathcal{X}\}$, i.e. all the actions only depend on the state of the system at each time epoch. For each stationary policy $\mathbf{z}$, the "one-step" transitions in the resulted Markov Chain $\left\{X_{k}^{\mathbf{z}}: k \geq 0\right\}$ are fully described by a "one-step" transition probability matrix:

$$
\mathbb{P}^{\mathbf{z}}:=\left(P_{X^{i}, X^{j}}^{\mathbf{z}}\right)_{\left\{X^{i}, X^{j} \in \mathcal{X},\right\}}
$$

where:

$$
P_{X^{i}, X^{j}}^{\mathbf{z}}=P\left(X_{k+1}=X^{j} \mid X_{k}=X^{i}, \mathbf{u}_{X^{i}, k}=\mathbf{u}_{X^{i}}\right)
$$

for all $k \in\{0,1,2, \ldots\}$, where $\mathbf{u}_{X^{i}}$ for any $X^{i} \in \mathcal{X}$ is determined according to the control policy z. $\mathbb{P}^{\mathbf{z}}$ is a stochastic matrix; that means $\mathbb{P}^{\mathbf{z}}$ is a square matrix of size $|\mathcal{X}| \times|\mathcal{X}|$, with its elements, $P_{X^{i}, X^{j}}^{\mathbf{z}}$, satisfying the following properties:

$$
\begin{aligned}
P_{X^{i}, X^{j}}^{\mathbf{z}} & \geq 0, \\
\sum_{j=1}^{|\mathcal{X}|} P_{X^{i}, X^{j}}^{\mathbf{z}} & =1,
\end{aligned}
$$

for all $i, j \in\{1, \cdots,|\mathcal{X}|\}$. For any integer number $n \geq 1$, the " $n$-step" transition matrix $\mathbb{P}^{\mathbf{z}, n}$ is as follows:

$$
\mathbb{P}^{\mathbf{z}, n}:=\left(P_{X^{i}, X^{j}}^{\mathbf{z}, n}\right)_{\left\{X^{i}, X^{j} \in \mathcal{X}\right\}}
$$

where:

$$
P_{X^{i}, X^{j}}^{\mathbf{z}, n}=P\left(X_{k+n}=X^{j} \mid X_{k}=X^{i}, \mathbf{u}_{X^{i}, k}=\mathbf{u}_{X^{i}}\right)
$$

for all $k \in\{0,1,2, \ldots\}$, where $\mathbf{u}_{X^{i}}$ for any $X^{i} \in \mathcal{X}$ is determined according to the control policy z. Using Chapman-Kolmogorov Equation [73], for stationary Markov 
Processes, results in the following equations:

$$
P_{X^{i}, X^{j}}^{\mathbf{z}, n+m}=\sum_{X^{k} \in \mathcal{X}} P_{X^{i}, X^{k}}^{\mathbf{z}, n} P_{X^{k}, X^{j}}^{\mathbf{z}, m}
$$

for all $n, m \in\{1,2, \ldots\}$ and all $X^{i}, X^{j} \in \mathcal{X}$. Equation (2.9) results in:

$$
\mathbb{P}^{\mathbf{z}, n+m}=\mathbb{P}^{\mathbf{z}, n} \times \mathbb{P}^{\mathbf{z}, m}
$$

\subsubsection{Stationary Distribution}

In a Markov Chain, states are classified according to some properties. In the sequel, we will introduce some of them, which we are going to use them later on in subsequent chapters.

\subsection{Irreducibility}

For any two states $X^{i}, X^{j} \in \mathcal{X}$, we say state $X^{j}$ is accessible from state $X^{i}$ if:

$$
P_{X^{i}, X^{j}}^{n}>0 ; \quad \text { for some } n \geq 0
$$

If $X^{i}$ is accessible from state $X^{j}$ and $X^{j}$ is accessible from state $X^{i}$, we say $X^{i}$ and $X^{j}$ communicate with each other and are in the same class, where a class is a subset of states in which all states can communicate with each other. The state space of a Markov chain is divided either into some disjoint classes or contains only one class. For the latter case, the Markov chain is called irreducible.

\subsection{Recurrence}

For any state $X \in \mathcal{X}$, we define:

$$
f_{X}=P\left(X_{k}=X, \text { for some } k \geq 1 \mid X_{0}=X\right)
$$

If $f_{X}=1$, then state $X$ is called recurrent. Otherwise, it is called transient. This property states that for a given recurrent state $X$, if $X_{0}=X$, then with probability 1 the Markov Chain will revisit this state. In addition, a state $X$ is called positive recurrent if starting from state $X$, the expected time until the process returns to this state is finite. If the state space $\mathcal{X}$ is finite, all recurrent states are positive recurrent. 


\subsection{Periodicity}

For a given state $X \in \mathcal{X}$, define:

$$
S_{X}=\left\{k: P_{X, X}^{k}>0, k \geq 1\right\}
$$

Let $d_{X}$ be the largest common denominator of the elements of $S_{X}$. If $d_{X}>1$, then state $X$ is called periodic. Otherwise, state $X$ is called aperiodic.

\subsection{Ergodicity and Stationary Distribution}

Positive recurrent and aperiodic states are called ergodic. Let $\left\{X_{k}: k \geq 0\right\}, k \in \mathbf{N}$ be an irreducible ergodic markov chain. Then, it can be shown that ( [73]) for any two states $X^{\prime}, X \in \mathcal{X}$ :

$$
\lim _{n \rightarrow \infty} P_{X^{\prime}, X}^{n}
$$

exists and is independent of $X^{\prime}$. Denote by $\pi(X)=\lim _{n \rightarrow \infty} P_{X^{\prime}, X}^{n}$, then $\pi=$ $\{\pi(X), \quad X \in \mathcal{X}\}$ is the unique non-negative solution of:

$$
\begin{aligned}
\pi(X) & =\sum_{X^{\prime} \in \mathcal{X}} \pi\left(X^{\prime}\right) P_{X^{\prime}, X} \\
\sum_{X \in \mathcal{X}} \pi(X) & =1
\end{aligned}
$$

That is the steady state probability distribution $\pi=\{\pi(X): \quad X \in \mathcal{X}\}$ exists [73][74], and is the solution of $\pi=\pi \mathbf{P}$ subject to $\pi \cdot \mathbf{1}=1$.

\subsubsection{Minimizing the infinite horizon Average Cost}

In Chapters 5 and 6, we introduce a model for a charging station of Electrical Vehicles (EVs). Our ultimate objective is to find a control policy minimizing the utilization cost of the charging station over an infinite time horizon.

Based on the assumptions presented in Chapters 5 and 6, the presented optimization problem can be modelled as a Continuos-time Markov Decision Process (MDP). Following standard practice in Markov Decision Processes, we will introduce a finite horizon $\delta$-discounted cost problem and find its optimal control policy. Then the optimal policy of the infinite horizon problem can be derived as a limit of the optimal 
policies of a sequence of $\delta$-discounted problems [75]. Let $\delta$ be the discount rate. Then given the initial state is $X$ and the time horizon is $\{t: t>0\}, \mathbf{J}_{t}^{\delta}(X)$, the minimum expected total $\delta$-discounted cost is defined as follows:

$$
\mathbf{J}_{t}^{\delta}(X)=\min _{\mathbf{z} \in \mathcal{Z}} \mathbf{E}_{\mathbf{X}}^{\mathbf{z}}\left(\int_{0}^{t} e^{-\delta \tau} C\left(X_{\tau}, \mathbf{u}_{X, \tau}\right) d \tau\right)
$$

Where $C\left(X_{\tau}, \mathbf{u}_{X, \tau}\right) d \tau$ is the cost incurred by the system at time $\tau$ for the duration of $d \tau$, when the system remains at state $X$ and the chosen action is $\mathbf{u}_{X, \tau}$. So, the infinite horizon optimal $\delta$-discounted expected cost is:

$$
\mathbf{J}^{\delta}(X)=\min _{\mathbf{z} \in \mathcal{Z}} \mathbf{E}_{\mathbf{X}}^{\mathbf{z}}\left(\int_{0}^{\infty} e^{-\delta \tau} C\left(X_{\tau}, \mathbf{u}_{X, \tau}\right) d \tau\right)
$$

If there exists a policy $\mathbf{z}^{*}$, resulting in $\mathbf{J}^{\delta}(X)$, this policy is called the optimal $\delta$ discounted expected cost policy. This optimal policy satisfies the following optimality condition [76]:

$$
\begin{aligned}
& \mathbf{J}_{t+d t}^{\delta}(X)=\min _{\mathbf{u}_{X, 0} \in \Omega}\left\{C\left(X, \mathbf{u}_{X, 0}\right) d t\right. \\
+ & \left.e^{-\delta d t} d t\left(\sum_{X^{\prime} \in \mathcal{X}} \mathbf{P}\left(X_{d t}=X^{\prime} \mid X_{0}=X, \mathbf{u}_{X, 0}\right) \mathbf{J}_{t}^{\delta}\left(X^{\prime}\right)\right)\right\}
\end{aligned}
$$

If the optimal policy is stationary, the optimal action for each state is independent of $t$ and only depends on the state of the system, $X$. So we have:

$$
\mathbf{z}^{*}=\left\{\mathbf{u}_{X, t}^{*}=\mathbf{u}_{X}^{*}: \quad X \in \mathcal{X}, \quad t\right\}
$$

To find the optimal policy, we first relate the continuous time Markov chain $\left\{X_{t}: t \geq 0\right\}$ to a suitable discrete time Markov chain. Then, we find the corresponding cost function and transition probabilities for the resulting discrete time Markov chain. Eventually, we will apply them to the corresponding discrete time dynamic programming equations to find the optimal action for each state and hence the optimal policy.

\subsubsection{Related Literature}

The most related works to our problem appears in the following studies: 
The model introduced in Chapters 5 and 6 is an extension of the one introduced in $[72,79,80]$. In these studies, a model for a charging station of EVs is presented. The main goal in [72] is to determine the proper size of the storage unit by comparing the simulation results of the charging station model for different sizes of the storage unit. The simulation results mainly focus on the average cost and the blocking probability of the station. In [79] and [80], the charging station model is extended to address charging of different classes of EVs, where EVs are classified with respect to their deployed batteries. This model is used in [79] to find out when to choose an energy storage with higher capacity over the one with a higher power rating and vice versa. Eventually, this model is used in [80] to find the optimal placing of a network of charging stations in an urban area. However, as it mentioned, in all of these works, only heuristic policies are deployed by each charging station, without trying to find optimal policies minimizing the operational cost of a charging station.

In [77], using Genetic Algorithm, an optimization policy is proposed in which authors considered different limitations in the systems, such as load on transformations, voltage limits and parking availability patterns. They showed using the proposed policy will result in reducing peak load and increasing the long-term utilization of power system elements. In [78], in the proposed charging scheduling policy, a routine-aware method is used in deciding whether a demanding PEV should be accepted or rejected in order to increase the charging station long-term benefit.

In [49], a discrete time Markov Decision Process (MDP) model for serving electrical demands is presented. In this model, it is assumed that the electrical demands can be fulfilled either by using the electrical supply from the grid or by drawing power from a local storage unit. In this model the demands arrive to the system according to a Poisson process, with identical but independent exponentially distributed random service times. In this model demands may not be blocked, but they can be delayed. The cost function is assumed to be a fixed convex function of consumed electrical power over the time. The goal in [49] is finding a control policy which minimizes the infinite horizon average cost of the system. Eventually an MDP problem is solved asymptotically for large storage capacity values.

In [81], authors introduce a model of serving electrical demands using power from either the grid or a local storage unit. The total amount of demands and the total available power from the grid is assumed to be bounded as well as the capacity of the storage unit. However, since the total electricity demand is assumed to be less than 
the available power from the grid, there are no blocked demands. The resulting model follows a discrete time MDP and the goal is to minimize the expected total discounted cost of serving demands. In [13], a dynamic programming based scheduling policy for a data center equipped with a local storage unit is devised. Taking into account the stochastic arrivals of network tasks, as well as characteristics of storage unit, the proposed policy will result in reducing the overall cost of the data center.

In Chapter 5, we will present a model for a charging station using a local storage energy unit with finite capacity. Then we will propose a control policy minimizing the operational cost of the charging station. In designing the scheduling policy, we will consider Real-Time Pricing scheme, in which the price of the electrical power is continuously being updated with respect to the state of the grid at each time instance. We model the charging station as a continuous time Markov Decision Process with three control actions: the probability of blocking new arrivals, the rate of charging the battery and the proportion of cars being served by the battery. Then by using dynamic programming, we will derive the optimal policy minimizing both discounted and average cost over an infinite time horizon.

\subsection{Summary}

In this chapter, we gave an overview of the background of our major works, and reviewed some of most related works in those areas. Especially we introduced Demand Side Management(DSM) and showed major works aiming at reducing either power peak or total convex cost of the grid. Then with respect to the model proposed in Chapters 3 and 4, Strip Packing Problem and major works in this area were investigated. In particular, we showed the differences between traditional Strip Packing, and the one we used to model the electrical demands. Eventually after emphasizing on the importance of deploying proper scheduling policies in charging stations of PHEVs, we reviewed some of the most related studies, aiming at designing scheduling policies minimizing the cost of charging stations. 


\section{Chapter 3}

\section{Power Strip Packing of Malleable Demands}

In this chapter, we consider a problem of supplying electricity to a set of customers in a smart-grid framework. Each customer requires a certain amount of electrical energy which has to be supplied during the time interval $[0,1]$. We assume that each demand has to be supplied without interruption, with possible duration between $\ell$ and $r$, which are given system parameters $(\ell \leq r)$. At each moment of time, the power of the grid is the sum of all the consumption rates for the demands being supplied at that moment. Our goal is to find an assignment that minimizes the power peak - maximal power over $[0,1]$ - while satisfying all the demands. To do this first we find the lower bound of the optimal power peak, for all possible values of malleability constraint pairs. We show that the problem depends on whether or not the pair $\ell, r$ belongs to a "Coverable" region $\mathcal{G}$. If it does - then an optimal assignment almost perfectly "fills" the rectangle time $\times$ power $=[0,1] \times[0, A]$ with $A$ being the sum of all the energy demands - thus achieving an optimal power peak $A$. Conversely, if $\ell, r$ do not belong to $\mathcal{G}$, we identify the lower bound $\bar{A}>A$ on the optimal value of power peak and introduce a linear time algorithm that almost perfectly arranges all the demands in a rectangle $[0, A / \bar{A}] \times[0, \bar{A}]$ and show that it is asymptotically optimal.

\section{$3.1 \quad$ Model}

Consider a set $\mathcal{N}=\{1,2, \ldots, n\}$ of energy demands $\left\{A_{i}, i \in \mathcal{N}\right\}$, needed to be scheduled in a finite time interval $[0,1]$. We assume that only "rectangular" shape of scheduling is permitted, meaning that each demand $i \in \mathcal{N}$ has to be supplied without interruption in some interval $\left[\tau_{i}, \tau_{i}+s_{i}\right]$ with a constant power intensity $d_{i}=\frac{A_{i}}{s_{i}}$. 
Obviously,

$$
0 \leq \tau_{i} \leq \tau_{i}+s_{i} \leq 1, \quad i \in \mathcal{N} .
$$

In addition to (3.1), we impose a demand malleability constraint. That is, we assume the power system has parameters $\ell$ and $r$ with $0 \leq \ell \leq r \leq 1$ so that

$$
\ell \leq s_{i} \leq r, \quad i \in \mathcal{N} .
$$

This is motivated by existence of electric appliances with flexibility on the charging rate. As a special application, we may think of a MicroGrid or a PEV parking lot with charging facilities where all customers either are known a priori or should register in advances.

A set of pairs $\pi=\left\{\left(\tau_{i}, s_{i}\right), i \in \mathcal{N}\right\}$, satisfying (3.1)-(3.2) for given parameters $(\ell, r)$, will be called a scheduling policy. Let $\Pi=\Pi^{(\ell, r)}$ be a set of all policies. For a policy $\pi \in \Pi$, we define its maximal power as

$$
P_{\max }^{\pi}=\max _{t \in[0,1]}\left\{P^{\pi}(t)\right\}
$$

where, for $\left(\tau_{i}, s_{i}\right) \in \pi$,

$$
P^{\pi}(t)=\sum_{i=1}^{n}\left(\frac{A_{i}}{s_{i}} \cdot 1_{\left\{\tau_{i} \leq t \leq \tau_{i}+s_{i}\right\}}\right), \quad 0 \leq t \leq 1 .
$$

We are interested in finding a scheduling that minimizes the peak to average ratio in the power grid, i.e. in finding

$$
P_{o p t}=\inf _{\pi \in \Pi} P_{m a x}^{\pi}
$$

Note that $P_{\text {opt }}$, although it is not stated explicitly, depends on the parameters $\ell$ and $r$.

Our setting resembles a so-called strip-packing problem [16] and [50]. Indeed by viewing the demands as rectangles, we want to pack them with their side $s_{i}$ parallel to horizontal axis in a rectangular bin of width $[0,1] \times P_{\text {opt }}$ where an optimal height $P_{\text {opt }}$ is unknown. The problem is known to be NP complete (see [52]) and therefore an optimal height cannot always be computed in polynomial time. 


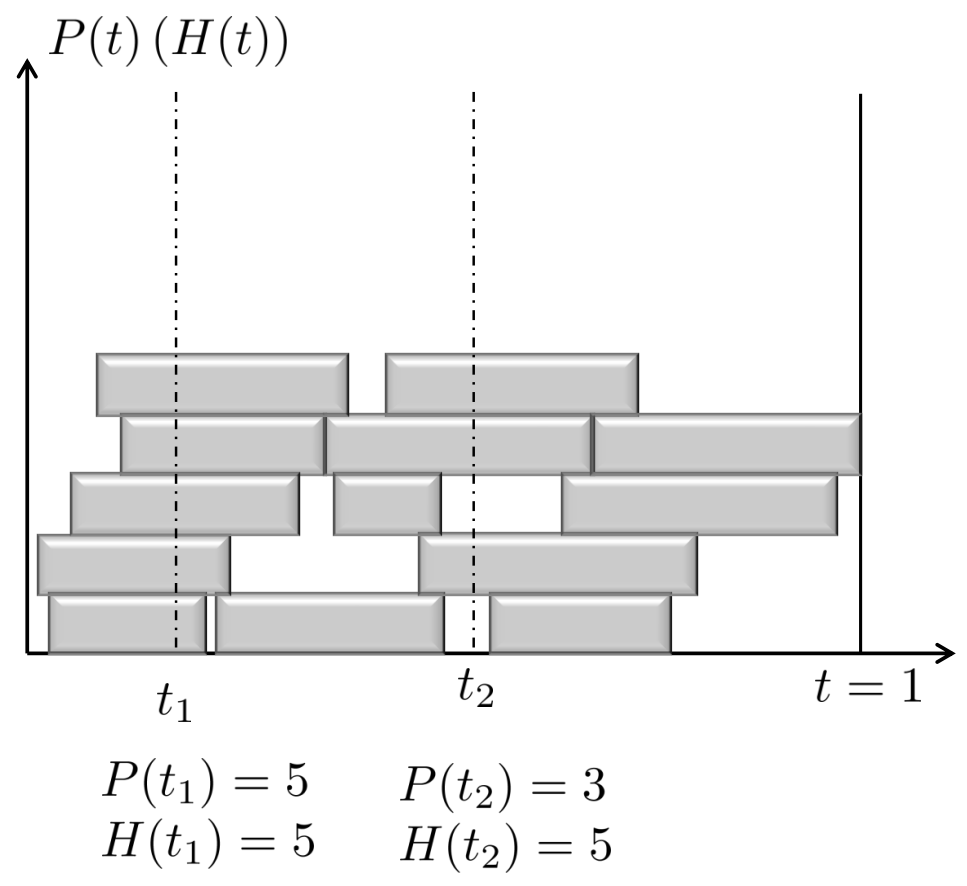

Figure 3.1: Different interpretations of power and height in PSP and TSP. For simplicity the height of each rectangle is assumed to be 1 . In PSP: $P\left(t_{1}\right)=5$ and $P\left(t_{2}\right)=3$ where in TSP $H\left(t_{1}\right)=H\left(t_{2}\right)=5$.

In the traditional strip packing (TSP) problem, however, the height at any time t $\left(H^{\pi}(t)\right)$ for a scheduling policy $\pi$ is defined as the uppermost boundary of scheduled rectangles at time $t$, while in our model (Power Strip Packing - PSP) the height of the strip packing is obtained from Equation (3.3). This difference arises from the nature of the electric power, in which the overall height (i.e. power) at any given time is the sum of the scheduled (i.e. active) demands (See Figure 3.1). Naturally, for any policy $\pi, P^{\pi}(t) \leq H^{\pi}(t)$ at any time $t$ and hence $P_{\max }^{\pi} \leq H_{\max }^{\pi}$.

\subsection{Main results}

We start with some preparatory work. Let $\ell$ and $r$ be fixed. A real number $w>0$ is called Coverable, if it can be represented as an integer combination of numbers from the interval $[\ell, r]$. That is, if there exist an integer number $q$ and a set of positive real values: $s_{1}, \ldots, s_{q} \in[\ell, r]$ such that $\sum_{i=1}^{q} s_{i}=w$.

Lemma 1 A value $w$ is coverable if and only if $\left\lceil\frac{w}{r}\right\rceil \leq \frac{w}{\ell}$. 


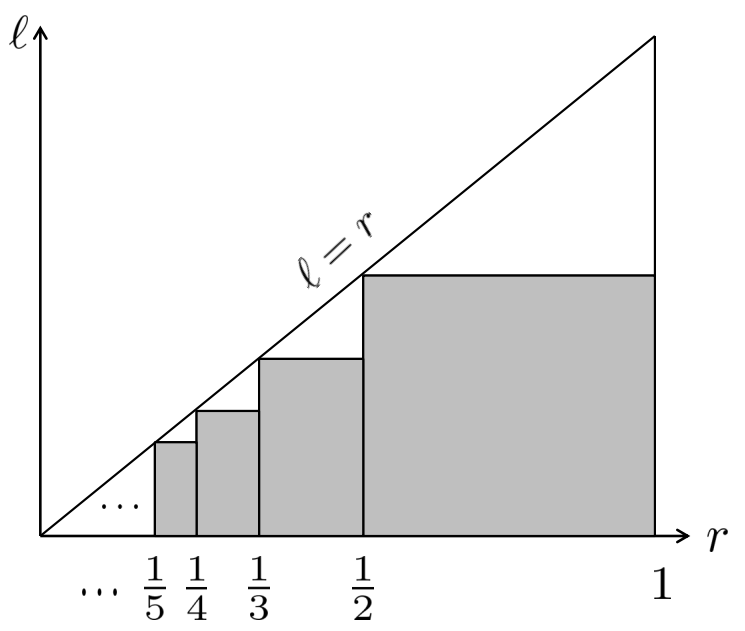

Figure 3.2: Coverable region vs. Non-Coverable region. The Coverable region is shown with shadow.

The proof is given in Appendix A.

Lemma 1 implies that the point $w=1$ is coverable iff $\left\lceil\frac{1}{r}\right\rceil \leq \frac{1}{\ell}$. Define a Coverable region to be the set of all pairs $(\ell, r)$ that makes $w=1$ a coverable point (see Fig. 3.2):

$$
\mathcal{G}=\{(\ell, r), \ell \leq r \text { and } 1 \text { is coverable }\}
$$

For any value $w$, we define its largest coverable point $w^{*} \triangleq w^{*}(w)$ as:

$$
w^{*} \triangleq \sup \{v: v \leq w, \text { such that } v \text { is coverable }\}
$$

Lemma 2 If $w$ is not coverable, then $w^{*}(w)=r \cdot\left\lfloor\frac{w}{r}\right\rfloor$.

The proof is given in Appendix B.

Using Lemma 2, if $(\ell, r) \notin \mathcal{G}$ and $Z=1$, then $Z^{*} \triangleq Z^{*}(1)=r \cdot\left\lfloor\frac{1}{r}\right\rfloor$. We are ready to state the main result of this chapter. 
Theorem 1 Let $A=\sum_{i=1}^{n} A_{i}$. Then $P_{\text {opt }} \in\left[\bar{A}, \bar{A}+\frac{A_{\max }}{\ell}\right]$, where

$$
\bar{A}= \begin{cases}A, & \text { if }(\ell, r) \in \mathcal{G}, \\ \frac{A}{Z^{*}} & , \text { if }(\ell, r) \notin \mathcal{G} .\end{cases}
$$

As the number of demands $n$ grows, the value of $\bar{A}$ grows as well, thus making the interval $\left[\bar{A}, \bar{A}+\frac{A_{\max }}{\ell}\right]$ relatively tight (relative to $\bar{A}$ ). Therefore, every scheduling policy $\pi$, whose corresponding value $P_{\max }^{\pi}$ belongs to that interval, will be considered asymptotically optimal. In the last section two of such policies will be introduced.

\subsection{Lower bound of $P_{\text {opt }}$}

Theorem 1 will be proved in two main steps, each dealing with lower and upper bounds respectively. Here we start our analyses by discussing about the lower bound.

\section{Lemma 3}

$$
P_{\text {opt }} \geq \bar{A}
$$

Proof.The Inequality (3.6) is obvious for $(\ell, r) \in \mathcal{G}$ (making $Z^{*}=1$ and $\bar{A}=A$ ). Therefore, in what follows we assume $(\ell, r) \notin \mathcal{G}$ and hence $Z^{*}<1$. To prove Lemma 3, we use the concept of the Fractional Strip Packing (FSP) [82], according to which it is allowed to perform horizontal cutting to each rectangle $\left(s_{i}, d_{i}\right)$ in order to get a set of equal width rectangles $\left\{\left(s_{i}, d_{i 1}\right),\left(s_{i}, d_{i 2}\right), \ldots,\left(s_{i}, d_{i Q_{i}}\right)\right\}$ such that $\sum_{j=1}^{Q_{i}} d_{i j}=d_{i}$ for all $i=1, \ldots, n$. Then, instead of packing the original set $\left\{\left(s_{i}, d_{i}\right) \quad i=1, \ldots, n\right\}$ of rectangles, a packing of a newly obtained set is performed.

Assume a certain set $\mathcal{N}$ of demands is given. For any FSP policy $\theta$, (that includes both assigning $\left(s_{i}, d_{i}\right), i \in \mathcal{N}$, as well as cutting), let $P_{\max }^{\theta}$ and $H_{\max }^{\theta}$ denote the maximal achieved heights, calculated according to power and traditional strip packing respectively. And let also $P_{o p t}^{F}$ and $H_{o p t}^{F}$ denote the FSP optimal values for power and traditional strip packing. Clearly, $A \leq P_{o p t}^{F} \leq H_{o p t}^{F}$ and also

$$
\begin{aligned}
& A \leq P_{o p t}^{F} \leq P_{o p t} \\
& A \leq H_{o p t}^{F} \leq H_{o p t}
\end{aligned}
$$


Next we will show that $\frac{A}{Z *} \leq P_{o p t}^{F}$, which, combined with (3.7), proves Lemma 3 .

Assume an arbitrary scheduling policy $\pi \in \Pi^{(\ell, r)}$ is given. Cut each demand $\left(s_{i}, d_{i}\right)$ horizontally to get the new set of very short height rectangles $\mathcal{N}^{\prime}=$ $\left\{\left(s_{i}, d_{i 1}\right),\left(s_{i}, d_{i 2}\right), \ldots,\left(s_{i}, d_{i Q_{i}}\right)\right\}$ such that $d_{i j}=\delta \forall i, j$ where $\delta$ is an infinitesimal positive real value. Now we try to pack these narrow rectangles in a strip of width 1 as follows: Let call an FSP policy $\theta^{*}$ as filling when there will not remain space to put any new rectangle with any arbitrary width $s_{i}\left(\ell \leq s_{i} \leq r\right)$ in each of its rows, i.e. the sum of all gaps in each row is always less than $\ell$. (maybe except for the last row). $\left(\Theta^{*}\right.$ is the set of all possible fillings $\left.\theta^{*}\right)$. Now, to pack all the narrow rectangle with a filling, we start with the first narrow rectangle and put it in the first row and then try to fill this row with subsequent narrow rectangles. After filling a row, we continue with filling subsequent rows until all the narrow rectangles will be scheduled.

\section{Lemma 4}

$$
P_{o p t}^{F}=\min _{\theta^{*} \in \Theta^{*}} P_{\max }^{\theta^{*}}
$$

The proof is given in Appendix C. So, with respect to Equation (3.9), in the sequel to show that $\frac{A}{Z *} \leq P_{\text {opt }}^{F}$ we just consider fillings $\theta^{*} \in \Theta^{*}$.

From Lemma 2, we know that in each row we cannot cover the time axis more than $Z^{*}$, then the maximum height (i.e. the upper boundary of the top row) cannot be less than $\frac{A}{Z^{*}}$, otherwise the total area will be less than $A$. Combining this fact with Inequality (3.8) results in:

$$
\frac{A}{Z^{*}} \leq H_{o p t}^{F} \leq H_{o p t}
$$

It proves Lemma 3 for TSP, but to prove this Lemma for PSP we should take into account all possible gaps in each row. Because, as mentioned before, the way of computing the maximum height in PSP is different from TSP. Indeed in PSP in calculating the height or more precisely the total power at each time, only the active demands at that time will be considered and gaps are not taken into account (Equation (3.3) and Figure 3.1). Also, note that by filling each row arbitrarily, these gaps can split in more than just one part in a row and may happen in different places of the time axis for different rows. Lemma 5 tells that in any arbitrary filling there will be exactly $K_{0}=\left\lfloor\frac{1}{r}\right\rfloor$ rectangles in each row and then in Lemma 6 it will be shown 
that for any arbitrary filling, there will be exactly $K_{0}$ equal length and identically placed "gap-free" interval in each row. Therefore, for each filling $\theta^{*}$, the resulted $P_{\max }$ here is indeed equal to $H_{\max }$ and consequently $P_{o p t}^{F}=H_{o p t}^{F}$. Eventually, using Lemma 4 together with (3.10) proves Lemma 3 for PSP.

Lemma 5 In any filling $\theta^{*}$ for a set of narrow rectangles of arbitrary widths $s_{i}$ (s.t. $\ell \leq s_{i} \leq r$ ), exactly $K_{0}=\left\lfloor\frac{1}{r}\right\rfloor=\left\lfloor\frac{1}{\ell}\right\rfloor$ narrow rectangles are needed (This may not apply for the last row which may contain less rectangles).

The proof is given in Appendix D.

Lemma 6 Under the same conditions as in Lemma 5, each row will contain $K_{0}$ identical (i.e. equal length and identically placed) intervals, $\left(1-\left(K_{0}-i+1\right) \cdot \ell, i \cdot \ell\right)$ for all $i=1, \ldots, K_{0}$, which cannot contain any gaps (maybe except for the last row).

Proof. Assume that we are trying to put $K_{0}$ arbitrary width narrow rectangles in a row. Now assume that we want to put the $i^{\text {th }}$ rectangle in this row. The largest value for the starting time of $i^{\text {th }}$ narrow rectangle, i.e. $\tau_{i}$, occurs when all the narrow rectangles starting after $\tau_{i}$, including $i^{\text {th }}$ rectangle itself, have the same width $\ell$. Also, all possible gaps happen before $\tau_{i}$. Then, the length of the remaining time after $\tau_{i}$ is: $\left(K_{0}-(i-1)\right) \cdot \ell$ and hence the largest value for $\tau_{i}$ is as follows:

$$
\tau_{i, \text { largest }}=1-\left(K_{0}-(i-1)\right) \cdot \ell
$$

On the other hand, the smallest value for the finishing time of this rectangle, i.e. $f_{i}=\tau_{i}+s_{i}$, happens only when this rectangle and all of the previous $(i-1)$ narrow rectangles have the same width $\ell$ and no gap placed in the time axis until after the $f_{i}$. Then the smallest value for $f_{i}$ is:

$$
f_{i, \text { smallest }}=i \cdot \ell
$$

Therefore, $i^{\text {th }}$ narrow rectangle starts at most at time $\tau_{i, \text { largest }}$ and remains active at least until $f_{i, s m a l l e s t}$. Then we conclude that the $i^{\text {th }}$ narrow rectangle (and only this one) is definitely active during the following time interval $\left(1-\left(K_{0}-i+1\right) \cdot \ell, i \cdot \ell\right)$ 
with the length:

$$
\begin{aligned}
& i \cdot \ell-\left(1-\left(K_{0}-i+1\right) \cdot \ell\right) \\
= & \left(K_{0}+1\right) \cdot \ell-1=\left\lceil\frac{1}{\ell}\right\rceil \cdot \ell-1>0
\end{aligned}
$$

Therefore, we have $K_{0}=\left\lfloor\frac{1}{r}\right\rfloor=\left\lfloor\frac{1}{\ell}\right\rfloor$ active intervals with equal non-zero length $\left(K_{0}+1\right) \cdot \ell-1$.

Note that, it is impossible for a demand to be completely placed in the interval between two consecutive active intervals, because the length of such an interval is $1-K_{0} \cdot \ell$ which is less than $\ell$ (because $1<\left\lceil\frac{1}{\ell}\right\rceil=\left(K_{0}+1\right) \cdot \ell$ ).

Corollary 1 In $i^{\text {th }}$ active interval of any filling $\theta^{*}$ (Lemma 6), when filling each row with narrow rectangles, definitely the $i^{\text {th }}$ narrow rectangle in that row is scheduled and the $i^{\text {th }}$ narrow rectangle is the only one which can be scheduled in this interval (maybe except for the last row).

Using Lemma 6, we conclude that in FSP when filling the rows with any arbitrary placement and with any arbitrary width $\left(s_{i}\right)$ for narrow rectangles, there will be exactly $K_{0}$ gap-free identical intervals in every row. Therefore the $P_{\max }^{\theta^{*}}$ is exactly the same as $H_{\text {max }}^{\theta^{*}}$ which is the sum of the heights of all the rows.

Note that no one can change FSP above and get the lower $P_{\max }^{\theta^{*}}$. For example if one tries to decrease the $P_{\max }^{\theta^{*}}$ by just one level, it needs to remove at least $K_{0}$ narrow rectangles from different gap-free intervals, either from one row or $K_{0}$ different rows (corollary 1). Then, these narrow rectangles will form at least one new row which then only can keep the total height the same as before and cannot decrease the $P_{\max }^{\theta^{*}}$. So, $P_{\max }^{\theta^{*}}$ above is in fact the minimum among all possible fillings and hence using Equation (3.9) in Lemma 4, it is equal to $P_{o p t}^{F}$.

From Lemma 2, we know that in a filling of narrow rectangles, in each row we cannot cover the time axis more than $Z^{*}=r \cdot\left\lfloor\frac{1}{r}\right\rfloor$, then the maximum height (i.e. the upper boundary of the top row) cannot be less than $\frac{A}{Z^{*}}$, otherwise the total area will be less than $A$. Therefore we have: $\frac{A}{Z^{*}} \leq P_{o p t}^{F} \leq P_{o p t}$ which proves Lemma 3.

In the next section we will use a simple linear time algorithm, in which we try to keep the $P(t)$ (and hence $P_{\max }$ ) around $\frac{A}{Z^{*}}$. Using this algorithm, we will show that the $P_{\text {opt }} \leq \bar{A}+\frac{A_{\max }}{\ell}$ which proves the upper bound of Theorem 1 and at the same time, it proves that this algorithm is asymptotically optimal. 


\subsection{Algorithm}

From Lemma 1, we know that $t=1$ is coverable if and only if $\left\lceil\frac{1}{r}\right\rceil \leq \frac{1}{\ell}$. Based on this fact, in the sequel, we will introduce different cases and in each case, we will find a proper policy which results in acceptable $P_{\max }$.

\subsubsection{Ideal Cases}

First, assume that $\ell \leq 1$ and $r=1$ (So 1 is coverable). In this case we have an optimal policy $\pi^{*}$ as follows: stretch the width of each demand to the whole time interval $[0,1]$, and then piling the demands up on top of each other. Another ideal case is when we have $\ell \leq \frac{A_{i}}{A} \leq r, i=1, \cdots, n$. In this case in optimal policy $\pi^{*}$, we have: $s_{i}=\frac{A_{i}}{A}$, therefore since $\sum_{i=1}^{n} s_{i}=\sum_{i=1}^{n} \frac{A_{i}}{A}=1,1$ is coverable. So in this case, we can simply stretch each intensity to $A$ as $A_{i}=\frac{A_{i}}{A} \times A$ and place the demands side by side. In both cases we have $P(t)=A$ for all $t \in[0,1]$ and hence:

$$
P_{\max }^{\pi^{*}}=A \leq \bar{A}+\frac{A_{\max }}{\ell}
$$

So, for ideal cases, we can pack the demands in a strip of height $A$ and because $A \leq P_{\text {opt }} \leq H_{\text {opt }}$, the output in this case is absolutely optimal.

\subsubsection{Near Ideal Cases}

In this case $t=1$ is coverable and hence $Z^{*}=1$. Then we perform scheduling policy $\pi$ as follows: Simply divide the time interval $[0,1]$ into $K_{0}=\left\lceil\frac{1}{r}\right\rceil$ non-overlapping time slots with equal length $S_{0}=\frac{1}{K_{0}}$ and since in this case, we have $\left\lceil\frac{1}{r}\right\rceil \leq \frac{1}{\ell}$ ( Lemma 1 ), we are sure that $\ell \leq S_{0} \leq r$.

Now, we can start with the first demand and put it in the first slot and continue this until the height of that slot becomes greater than or equal to the threshold value, $A$, then continue by putting the next demand in the next slot and doing the same procedure until all of the demands have been packed. Therefore, the height of each slot is at most $A+\frac{A_{\max }}{S_{0}}$. So, in this case we have:

$$
P_{\max }^{\pi} \leq A+\frac{A_{\max }}{S_{0}} \leq \bar{A}+\frac{A_{\max }}{\ell}
$$




\subsubsection{Non-Ideal Cases}

In this case $t=1$ is NOT coverable. So, the constraints on $\ell$ and $r$ are as follows:

$$
0<\ell<r<1 \quad \text { and } \quad\left\lceil\frac{1}{r}\right\rceil>\frac{1}{\ell}
$$

These conditions imply that $\left\lfloor\frac{1}{r}\right\rfloor=\left\lfloor\frac{1}{\ell}\right\rfloor$. Using Lemma 2, we have: $Z^{*}=r \cdot\left\lfloor\frac{1}{r}\right\rfloor<1$.

In this case, the chosen policy $\pi$ is as follows: We simply divide the time interval $\left[0, Z^{*}\right]$ into $K_{0}=\left\lfloor\frac{1}{r}\right\rfloor$ non-overlapping time slots with equal length $r$ and leave the remaining part of the time interval, i.e. $\left[Z^{*}, 1\right]$ unscheduled. Now, we can use the same approach used in Subsection 3.4.2, while here $s_{i}=S_{0}=r, \quad i=1, \ldots, n$ and $K_{0}=\left\lfloor\frac{1}{r}\right\rfloor$ and also the threshold value is $\frac{A}{Z^{*}}$. Then in this case we have:

$$
P_{\max }^{\pi} \leq \frac{A}{Z^{*}}+\frac{A_{\max }}{r} \leq \bar{A}+\frac{A_{\max }}{\ell}
$$

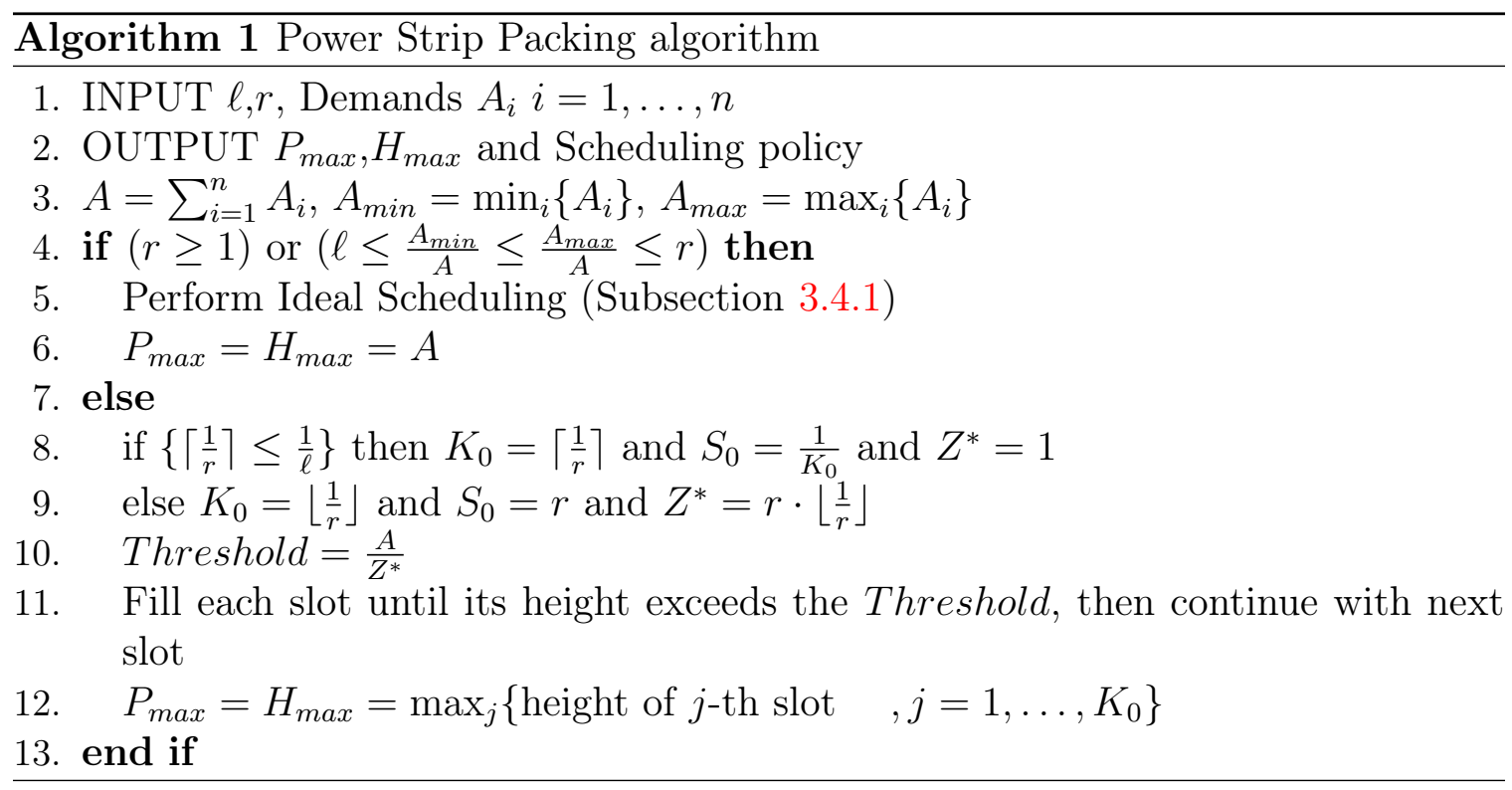




\subsection{Proof of Theorem 1}

Proof. Lemma 3 showed that $\bar{A} \leq P_{\text {opt }}$, which proves the lower bound part of Theorem 1. In addition, in Section 3.4, for each set of demands $\mathcal{N}$ with pair $(\ell, r)$ resulting in ideal, near-ideal, non-ideal cases, we introduced a policy $\pi$ with performance $P_{\max }^{\pi} \leq \bar{A}+\frac{A_{\max }}{\ell}$ which results in:

$$
P_{\text {opt }} \leq \bar{A}+\frac{A_{\max }}{\ell}
$$

which proves the upper bound part of Theorem 1. Therefore combining inequalities (3.6) and (3.14) gives us: $P_{\text {opt }} \in\left[\bar{A}, \bar{A}+\frac{A_{\max }}{\ell}\right]$ which proves Theorem 1.

\subsection{Computational Results}

Algorithm 1, which is called "Power Strip Packing" algorithm, summarizes all the steps of packing the demands. Clearly, the running time of this algorithm is linear in $n$, the number of demands. As we discussed in Section 3.4, we showed for this algorithm we have:

$$
P_{\max }^{P S P} \leq \bar{A}+\frac{A_{\max }}{\ell} \leq P_{o p t}+\frac{A_{\max }}{\ell}
$$

In obtaining the last inequality in (3.15), we used Inequality (3.6) in Lemma 3. So, as it was mentioned in Chapter 2, Subsection 2.2, it corresponds to asymptotic performance ratio being exactly 1 . Therefore, this algorithm is asymptotically optimal for all different cases. Furthermore, as we can see in Algorithm 1, this algorithm is linear time.

We can achieve better performance in terms of flatter $P(t)$ for $t \in[0,1]$ and get lower $P_{\max }$, albeit at the expense of increasing the number of operations. For example, the greedy algorithm works by ordering the demands by non-increasing areas $\left(A_{i}\right)$ and select $s_{i}=S_{0}$ for $i=1, \cdots, n$ exactly the same as that of in PSP Algorithm 1 (and hence $d_{i}=\frac{A_{i}}{S_{0}}$ ). Then start with the first (i.e. largest) demand and put it in a slot with minimum total height and continue this for subsequent demands until packing all of the demands. Note that when greedy algorithm place a new demand in a slot, it is not possible that the height of every slot being greater than $\frac{A}{Z^{*}}$, otherwise the 


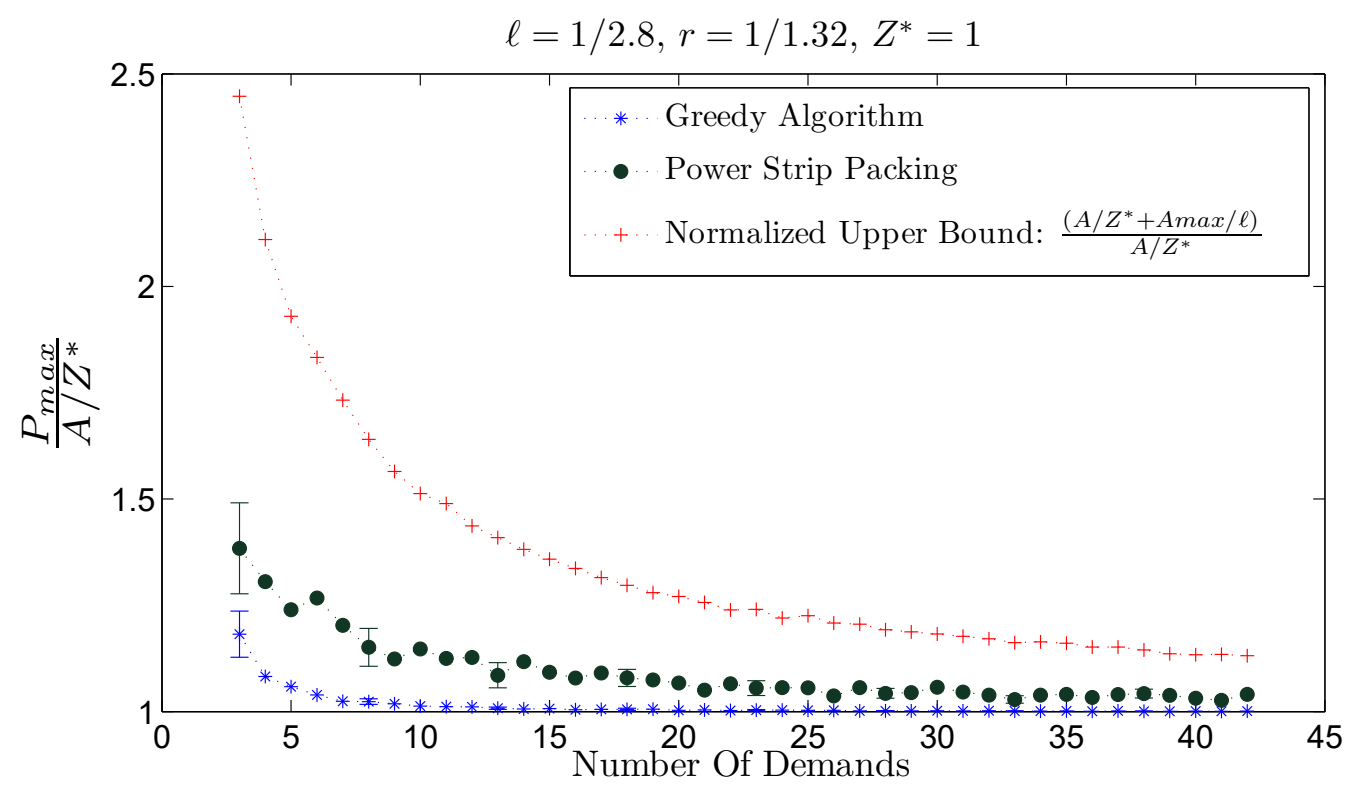

Figure 3.3: Performance of the algorithms for Near-Ideal case with respect to Peak criteria for different number of demands, where malleability constraint pair is in the coverable region $\mathcal{G}(\ell=0.35714$ and $r=0.75758)$, that is $Z^{*}=1$.

total area $\frac{A}{Z^{*}} \times Z^{*}$ exceeds $A$, the sum of the demands. Therefore the height of each slot is at most $\frac{A}{Z^{*}}+\frac{A_{\max }}{S_{0}}$. So, the inequalities (3.12) and (3.13) are still valid and hence it is also asymptotically optimal. However, the running time is $O\left(n^{2}\right)$.

Figures 3.3 and 3.4 illustrate the outputs of the presented algorithms (Algorithm 1 and the greedy algorithm) for different values of $|\mathcal{N}|$, the number of demands, in near ideal cases and non-ideal cases respectively. In these figures, the demands, i.e. $A_{i}$ 's, are independent and identically uniformly distributed in the interval $[0, \ell]$. For each value of $|\mathcal{N}|$, each of the algorithms is performed 30 times and then the mean value is depicted as the corresponding $P_{\max }$ of each algorithm. Furthermore, for some values of $|\mathcal{N}|$ the corresponding $0.95 \%$ confidence intervals are shown in these figures.

As it can be seen from these figures, in both cases, with or without ordering always we have: $P_{\max } \leq \frac{A}{Z^{*}}+\frac{A_{\max }}{\ell}$. However, as we can see in these figures, ordering can improve the performance of the algorithm albeit at the expense of increasing the number of operations. 


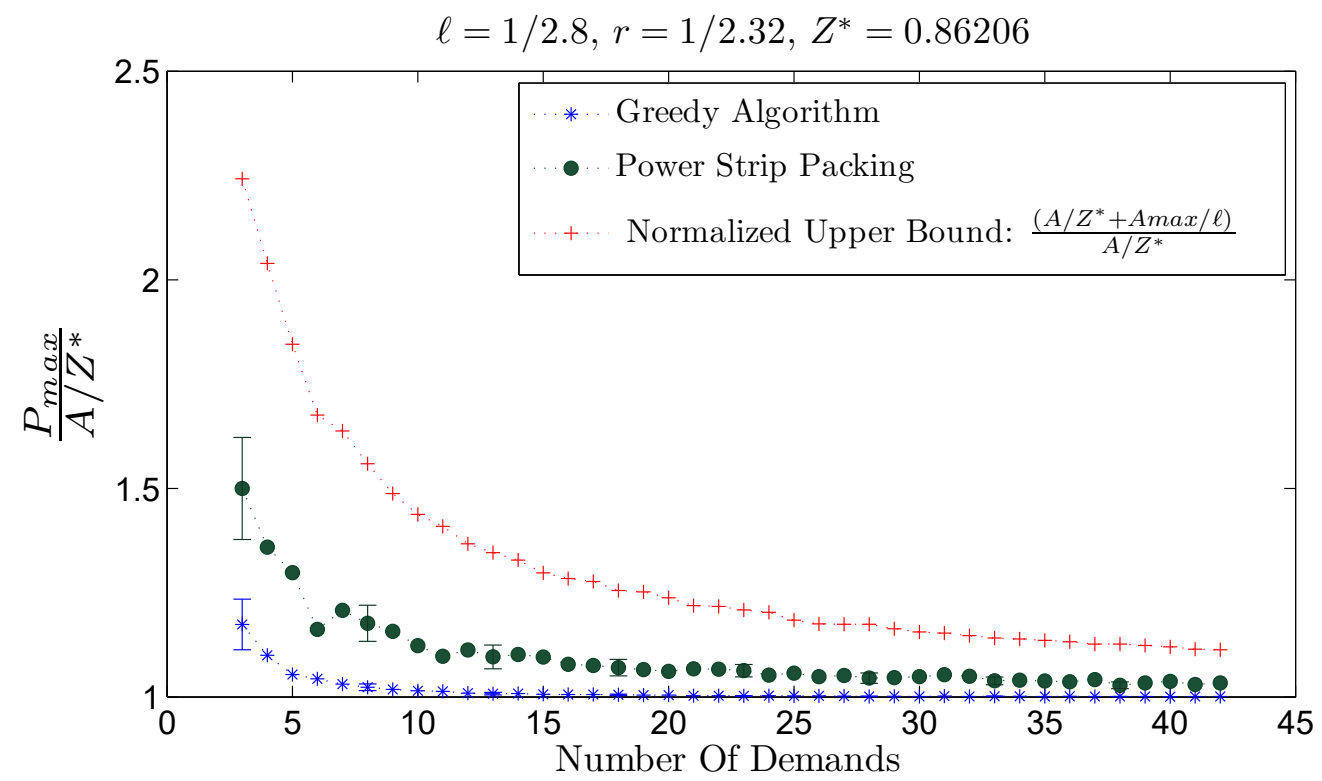

Figure 3.4: Performance of the algorithms for Non-Ideal case with respect to Peak criteria for different number of demands, where malleability constraint pair is NOT in the coverable region $\mathcal{G}(\ell=0.3571$ and $r=0.43103)$, that is $Z^{*}<1$.

\subsection{Summary}

In this chapter, considering a problem of supplying electricity to malleable demands, we introduced Power Strip Packing (PSP) problem. We assumed that each energy demand has to be supplied during the finite time interval $[0,1]$, without interruption, with possible duration between $\ell$ and $r$. We aimed at finding an assignment that minimizes the power peak - maximal power over $[0,1]$ - while satisfying all the demands. Therefore, after finding the lower bound of optimal power peak, we showed that using a linear time algorithm will result in asymptotically optimal performance. In the next chapter, we will extend this problem by letting each demand have its own malleability constraints pair. 


\section{Chapter 4}

\section{Asymptotic Convex Optimization for Packing Random Malleable Demands}

In this chapter, we extend the problem of scheduling malleable electric demands, which we studied in the previous chapter. Here, we consider an asymptotic setting with a large number of relatively small energy demands. Such a setting provides several major generalizations over the previous chapter. Firstly, each demand $i \in \mathcal{N}$ will now have its own scheduling constraints $\left(\ell_{i}, r_{i}\right)$, as an opposite to a unique pair $(\ell, r)$ from (3.2) - thus replacing the condition (3.2) by the following more generic condition:

$$
\ell_{i} \leq s_{i} \leq r_{i}, \quad i \in \mathcal{N}
$$

Secondly, the triples $\left(A_{i}, \ell_{i}, r_{i}\right), i \in \mathcal{N}$ will become random i.i.d. vectors, with a certain distribution of random pairs $\left(\ell_{i}, r_{i}\right)$, where $A_{i}$ is the amount of energy, while $\ell_{i}$ and $r_{i}$ are respectively, the left and right constraints on the length of the time period, during which $A_{i}$ has to be supplied without interruption. Moreover, we will also be able to include a convex cost in the treatment. Therefore the objective will be finding a scheduling policy minimizing the power peak - maximal power over $[0,1]$ - and/or the operational convex cost of the system while satisfying all the demands. The problem becomes very complicated as the number $n$ of demands increases. To address this issue, we consider an asymptotic approach, in which the average amount of energy in each demand is inversely proportional to $n$, thus keeping the total scheduled amount stable.

The main contributions in this chapter can be summarized as follows: 


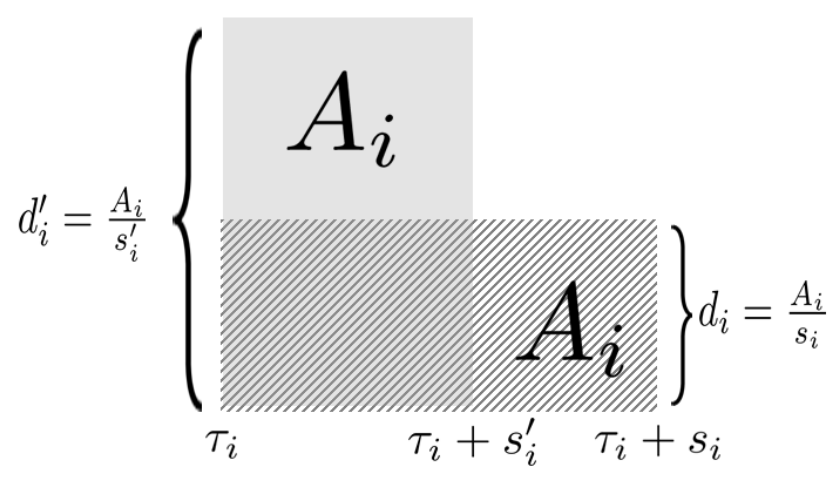

Figure 4.1: An example of a Malleable Rectangular-Shape energy demand, with energy requirement $A_{i}$ and malleability constraints $\left(\ell_{i}, r_{i}\right)$. In this example, this energy demand is served in two different ways: $\left(\tau_{i}, s_{i}\right)$ and $\left(\tau_{i}, s_{i}^{\prime}\right)$. The service times $s_{i}$ and $s_{i}^{\prime}$ satisfy $0<\ell_{i} \leq s_{i}^{\prime}<s_{i} \leq r_{i} \leq 1$.

We propose a new model to represent a grid composed of electrical "rectangularshape" demands with stochastic malleability constraints; that is the service duration of each demand has to satisfy given malleability constraints. We will consider a very wide range of probability distributions of malleability constraints and energy requirements in our settings. For this general setting, we will propose a linear-time scheduling policy. We will show that it is asymptotically optimal with respect to two cost criteria: the maximum power and the total convex cost of power consumption. Eventually, we will extend our approach for cases of demand scheduling in an arbitrary length time horizon, in which demands are arriving randomly during the scheduling horizon.

In this chapter, first we introduce lower bounds for both types of costs and then introduce a scheduling algorithm, asymptotically optimal in the sense that its cost converges to a corresponding lower bound almost surely, as $n$ increases to infinity. Moreover, the algorithm is on-line (each demand is scheduled at the time its parameters become known) and has fully linear running time.

\subsection{Model}

Here we are proposing our model for an electrical grid composed of $n$ "Malleable Rectangular-Shape" energy demands. This model is inspired by considering a MicroGrid [2] or a charging facility for Plug-in Hybrid Electric Vehicles (PHEVs), where 
energy requirements are known and present prior to the beginning of scheduling all these requirements. In our model, a set of $n$ energy demands needs to be scheduled in the interval $[0,1]$ in such a way that: 1) Each demand has to be supplied without interruption, and with constant intensity; 2) The duration of its supply must satisfy left and right constraints, called malleability constraints. Each demand comes with its own malleability constraints.

Thus, the main component of our model is a set of random triples $\left\{\left(A_{i}, \ell_{i}, r_{i}\right), i=\right.$ $1, \ldots, n\}$, where $A_{i} \in \mathbf{R}^{+}$represents the amount of energy of $i^{t h}$ demand and the pair $\left(\ell_{i}, r_{i}\right)$ - the malleability constraints for $i^{\text {th }}$ demand $\left(0<\ell_{i} \leq r_{i} \leq 1\right)$.

The objective of the controller is to optimally (in a certain sense, to be specified later) schedule the demands while respecting the malleability constraints. Formally let $\tau_{i}$ be the starting time of serving the $i^{\text {th }}$ demand, and $s_{i}$ be its duration of service. The objective of the controller is to determine a scheduling policy $\pi=\left\{\left(\tau_{i}, s_{i}\right), i=1, . ., n\right\}$, (Figure 4.1), such that:

- The demand $A_{i}$ is being supplied during time interval $\left[\tau_{i}, \tau_{i}+s_{i}\right] \subset[0,1]$, at a constant power level $A_{i} / s_{i}$,

- Its service duration, $s_{i}$, has to satisfy given left and right malleability constraints $\left\{\left(\ell_{i}, r_{i}\right), i=1, \ldots, n\right\}$ as follows:

$$
\ell_{i} \leq s_{i} \leq r_{i}, \quad i=1, \ldots, n
$$

The triples of demands and constraints $\left\{\left(A_{i}, \ell_{i}, r_{i}\right), i=1, \ldots, n\right\}$ are assumed to be random vectors, independently and identically distributed based on some probability distribution, to be discussed in Section 4.2.1.

Taking into account the malleability for electrical demands, is mainly motivated by the presence of multi-rate chargeable electrical devices. In such devices, charging rates are variable, whereas the energy requirements are fixed. For example, slow and fast charging options are available in many electrical devices. Drawing different electrical current amounts(Amps), and consequently different power consumption(Watts), results in different charging time(Seconds) to meet the energy requirement(Jules) of the electrical device. As another example, we could mention heating devices such as microwaves. In these devices, by changing the heat level, which is directly proportional to the power consumption, we could lengthen or shorten the duration of the 
heating process. These are examples of devices with the option of consuming specific amounts of energy with different possible rates and consequently durations.

At each time instance, the power consumption of the grid is the sum of all the power consumption of the demands being served at that moment. Then for a policy $\pi$,

$$
P^{\pi, n}(t)=\sum_{i=1}^{n}\left(\frac{A_{i}}{s_{i}} \cdot 1_{\left\{\tau_{i} \leq t \leq \tau_{i}+s_{i}\right\}}\right)
$$

is the total power consumption of the system at time $t \in[0,1]$. We are interested in the optimization problems with respect to two different cost functions, namely, the maximum power cost

$$
P_{\max }^{\pi, n}=\max _{t \in[0,1]}\left\{P^{\pi, n}(t)\right\}
$$

and the total convex cost of power consumption, defined, for a given convex function $h$, as

$$
C^{\pi, n}=\int_{t=0}^{1} h\left(P^{\pi, n}(t)\right) d t
$$

Even though these two costs are related to each other, they are not exactly the same. In fact, each additional power unit needed to serve demands becomes more expensive as the total power demand increases [83]. Therefore, the cost of power consumption is being considered as a convex function of instantaneous energy consumption rate. Our goal is to design scheduling policies minimizing the operational cost of a given electrical grid, with respect to these two cost criteria.

\subsection{Cost Optimization. The Main Result}

With respect to our assumptions in Section 4.1, we are interested to find a scheduling policy $\pi$, minimizing operational costs $P_{\max }^{\pi, n}$ and $C^{\pi, n}$ (Equations (4.4) and (4.5) respectively). An optimal (in any sense) scheduling of the demands is generally known to be NP complete (see [52]) and therefore cannot always be determined in polynomial time. In what follows we consider an asymptotic approach, in which the number $n$ of the demands is large, and the sizes of demands are inversely proportional to $n$. 
(The size of a demand is represented by the area of the rectangle characterizing the demand).

Practical cases may correspond to charging facilities for Plug-in Hybrid Electric Vehicles (PHEVs) or a MicroGrid with a significantly large amount of relatively much smaller demands.

\subsubsection{Asymptotic Setting on a Grid}

Assumption 1 Let $\left\{\xi_{i}, i=1, \ldots, n\right\}$ be a sequence of i.i.d. random variables with $\alpha:=E\left(\xi_{i}\right)<\infty$ and $\operatorname{Var}\left(\xi_{i}\right)<\infty$. Then for the demand size distribution, we

assume $A_{i} \stackrel{d}{=} \xi_{i} / q_{n}$, where $\left\{q_{n}\right\}$ is an increasing sequence, satisfying $n / q_{n} \rightarrow \lambda$ as $n \rightarrow \infty$, for some $\lambda \in(0, \infty)$.

In other words $q_{n}$ is a scaling parameter of order $n$. Essentially it means we have $n$ demands, each one of order $\left(\frac{1}{n}\right)$.

The next assumption addresses the distribution of the malleability constraints. Let $d \in \mathbb{N}$ be given and define $\Omega_{d}$, the set of all possible values for malleability constraint pair $\left(\ell_{i}, r_{i}\right)$, as follows:

$$
\Omega_{d}=\left\{\left(\frac{\gamma}{d}, \frac{\beta}{d}\right): \gamma, \beta \in\{1, \ldots, d\}, \gamma \leq \beta\right\}
$$

In [36], we considered a special case, where the pairs of malleability constraints, $\left(\ell_{i}, r_{i}\right)$, are independently and identically distributed with a uniform distribution in $\Omega_{d}$. We extend this assumption by considering more general conditions for the distribution of malleability constraint pairs, $\left(\ell_{i}, r_{i}\right)$.

Assumption 2 The random pairs $\left\{\left(\ell_{i}, r_{i}\right)\right\}, i \in\{1, . ., n\}$ are independently and identically distributed in $\Omega_{d}$ according to the following law:

- The value of the right constraint $r_{i}$ is assumed to have an arbitrary discrete distribution in $\left\{\frac{1}{d}, \frac{2}{d}, \ldots, 1\right\}$, with:

$$
p_{k}:=\operatorname{Prob}\left(r_{i}=\frac{k}{d}\right)
$$


- given $r_{i}=k / d$, the left constraint $\ell_{i}$ is uniformly distributed in $\left\{\frac{1}{d}, \ldots, \frac{k}{d}\right\}$, that is for $k^{\prime} \leq k$ :

$$
\operatorname{Prob}\left(\ell_{i} \leq \frac{k^{\prime}}{d} \mid r_{i}=\frac{k}{d}\right)=\frac{k^{\prime}}{k}
$$

\subsubsection{Optimal Policy}

Let $\Pi$ be the set of all possible scheduling policies, defined in Section 4.1. For a policy $\pi \in \Pi$, let

$$
P^{\pi, n}(t)=\sum_{i=1}^{n}\left(\frac{A_{i}}{s_{i}} \cdot 1_{\left\{\tau_{i} \leq t \leq \tau_{i}+s_{i}\right\}}\right)
$$

be the total power demand for the system at time $t \in[0,1]$. In the sequel, we are interested in the optimization problems of scheduling the demands with respect to two cost criteria described by Equations (4.4) and (4.5). Their corresponding optimal values are computed as follows:

$$
\begin{gathered}
P_{o p t}^{n}=\inf _{\pi \in \Pi} P_{m a x}^{\pi, n}=\inf _{\pi \in \Pi}\left(\max _{t \in[0,1]}\left\{P^{\pi, n}(t)\right\}\right), \\
C_{o p t}^{n}=\inf _{\pi \in \Pi} C^{\pi, n}=\inf _{\pi \in \Pi}\left(\int_{t=0}^{1} h\left(P^{\pi, n}(t)\right) d t\right) .
\end{gathered}
$$

Define

$$
L_{P}^{n}=\sum_{i=1}^{n} A_{i} \quad \text { and } \quad L_{C}^{n}=h\left(\sum_{i=1}^{n} A_{i}\right) .
$$

It is easy to see that the above quantities provide the lower bounds for $P_{o p t}^{n}$ and $C_{o p t}^{n}$ respectively. It can be easily seen that:

$$
P_{o p t}^{n} \geq \sum_{i=1}^{n} A_{i}=L_{P}^{n}
$$


since all demands are scheduled in $[0,1]$. For the second bound, we use Jensen's inequality [84]:

$$
\int_{t=0}^{1} h\left(P^{\pi, n}(t)\right) d t \geq h\left(\int_{t=0}^{1} P^{\pi, n}(t) d t\right)
$$

to get

$$
C_{o p t}^{n} \geq h\left(\int_{t=0}^{1} P^{\pi, n}(t) d t\right)=h\left(\sum_{i=1}^{n} A_{i}\right)=L_{C}^{n} .
$$

From the Law of Large Numbers and Assumption 1:

$$
\sum_{i=1}^{n} A_{i}=\sum_{i=1}^{n} \frac{\xi_{i}}{q_{n}} \rightarrow \lambda \alpha \text { a.s. } n \rightarrow \infty,
$$

and therefore:

$$
\left\{\begin{array}{l}
L_{P}^{n} \rightarrow \lambda \alpha \\
L_{C}^{n} \rightarrow h(\lambda \alpha)
\end{array} \quad \text { a.s. } n \rightarrow \infty,\right.
$$

We are ready to state the main result of the section.

Theorem 2 Given Assumptions 1, 2 (Subsection 4.2.1) hold. Algorithm 2 (the Covering policy $\mathbf{v}^{*}$ ) is asymptotically optimal, in the sense that the costs (4.10), (4.11) approach their respective lower bounds (4.12)

$$
\begin{aligned}
\text { a.s. } & \lim _{n \rightarrow \infty}\left[\max _{t \in[0,1]}\left\{P^{\mathbf{v}^{*}, n}(t)\right\}-L_{P}^{n}\right]=0 \\
\text { a.s. } & \lim _{n \rightarrow \infty}\left[\int_{t=0}^{1} h\left(P^{\mathbf{v}^{*}, n}(t)\right) d t-L_{C}^{n}\right]=0
\end{aligned}
$$

\subsubsection{Discussion of the Algorithm}

The main idea of the Algorithm 2 is to approximately achieve the same total power intensity throughout the interval $[0,1]$. For each $r_{i}=k / d$, the time interval $[0,1]$ is 


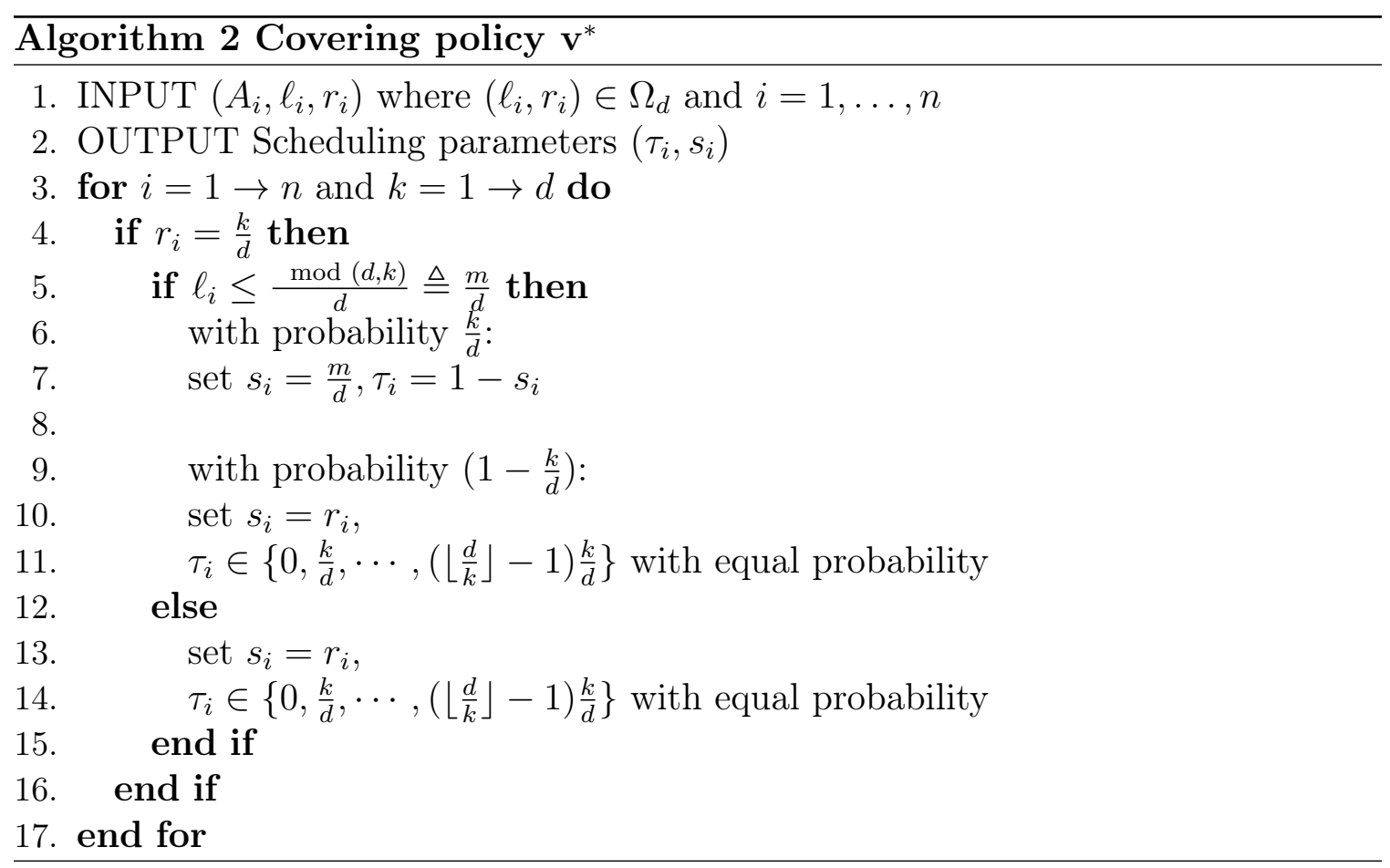

divided into $\lceil d / k\rceil$ time slots. Out of them, $\lfloor d / k\rfloor$ slots will have equal width $k / d$; if $\bmod (d, k) \neq 0$ the last time slot will have width $\frac{\bmod (d, k)}{d}$. Subsequently, all the demands with $r_{i}=k / d$ are distributed into these time slots in such a manner that the total power intensity is approximately the same among all the slots. The next section provides a detailed proof.

Figures 4.2 and 4.3 illustrate the scheduling of malleable demands using the covering policy $\mathbf{v}^{*}$ for $d=8$. In particular, Figure 4.2 illustrates the scheduling of demands with their right malleability constraint equal to $\frac{3}{8}$. As illustrated in this figure, six demands with $r_{i}=\frac{3}{8}$ are scheduled in each slot of width $s_{i}=r_{i}=\frac{3}{8}$, while the power intensity of each of these demands is $\frac{d}{k}=\frac{8}{3}$ times its energy demand, $A_{i}$. On the other hand, since $\bmod (8,3) \neq 0$, the width of the last slot is equal to $\frac{\bmod (d, k)}{d}=\frac{2}{8}$. Then in this slot, the intensity of each demand is $\frac{d}{\bmod (d, k)}=\frac{8}{2}$ times its energy demand $A_{i}$. In this figure, four demands with $r_{i}=\frac{3}{8}$ will be scheduled in this slot. Consequently, the total power intensity in each of these time slots will be kept close to the one in the other slots. In the presence of a large number of demands, on average, we expect that $\frac{3}{8}$ of demands with $r_{i}=\frac{3}{8}$ to be scheduled in each slot of width $s_{i}=r_{i}=\frac{3}{8}$ and $\frac{\bmod (d, k)}{d}=\frac{2}{8}$ of them in the slot of width $\frac{\bmod (d, k)}{d}=\frac{2}{8}$. Note that Assumption 2 (Equation 4.8) assures us that there are enough demands to be scheduled in the last 


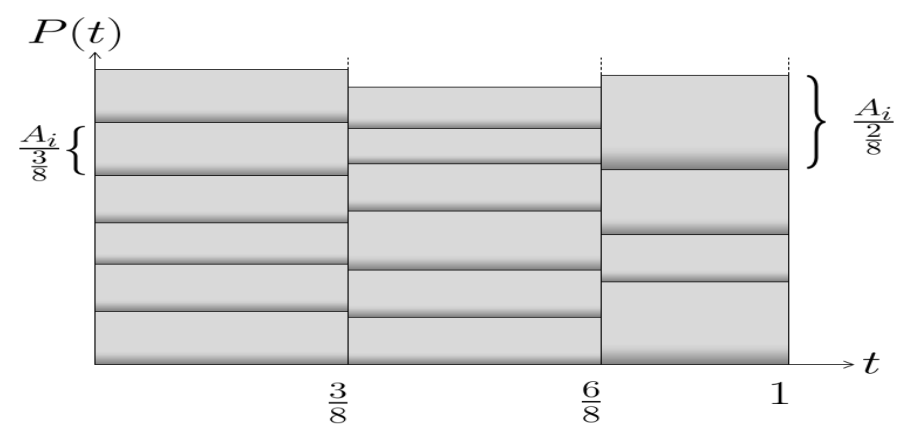

Figure 4.2: Applying the covering policy $\mathbf{v}^{*}$ for $d=8$ and $r_{i}=\frac{3}{8}$.

slot. According to this assumption, for demands with $r_{i}=\frac{3}{8}$, around $\frac{m}{k}=\frac{2}{3}$ of them have left malleability constraint satisfying the condition $\ell_{i} \leq \frac{2}{8}$ (while only $\frac{m}{d}=\frac{2}{8}$ of them are needed to be scheduled in the slot with its width equal to $\frac{2}{8}$ ). Figure 4.3 illustrates the output of the covering policy $\mathbf{v}^{*}$ for all possible values of $r_{i}$ where $r_{i}=\frac{k}{8}, \quad k=1, \cdots, d$, and $d=8$.

\subsubsection{Proof of Theorem 2}

We will show the a.s. convergence

$$
\max _{0 \leq t \leq 1}\left|P^{\mathbf{v}^{*}, n}(t)-\lambda \alpha\right| \rightarrow 0, \quad \text { a.s. } n \rightarrow \infty,
$$

Then Theorem 2 will follow directly from (4.17) and (4.20) and the continuity of convex function $h($.$) .$

To show (4.20) we start by decomposing the set of all scheduled demands into $d \times\lceil d / k\rceil$ disjoint groups: for $1 \leq k \leq d, 1 \leq j \leq\lceil d / k\rceil$

$$
Q_{k, j}^{n}=\left\{i \in\{1, . ., n\} \quad: \quad r_{i}=\frac{k}{d}, \quad \tau_{i}=(j-1) \frac{k}{d}\right\} .
$$

That is $Q_{k, j}^{n}$ is the set of demands with $r_{i}=\frac{k}{d}$, scheduled in the time interval [ $(j-$ 1) $\left.\frac{k}{d}, \min \left(j \frac{k}{d}, 1\right)\right]$. Let

$$
P_{k, j}^{n}=\sum_{i \in Q_{k, j}^{n}} \frac{A_{i}}{s_{i}}, \quad 1 \leq j \leq\lceil d / k\rceil .
$$




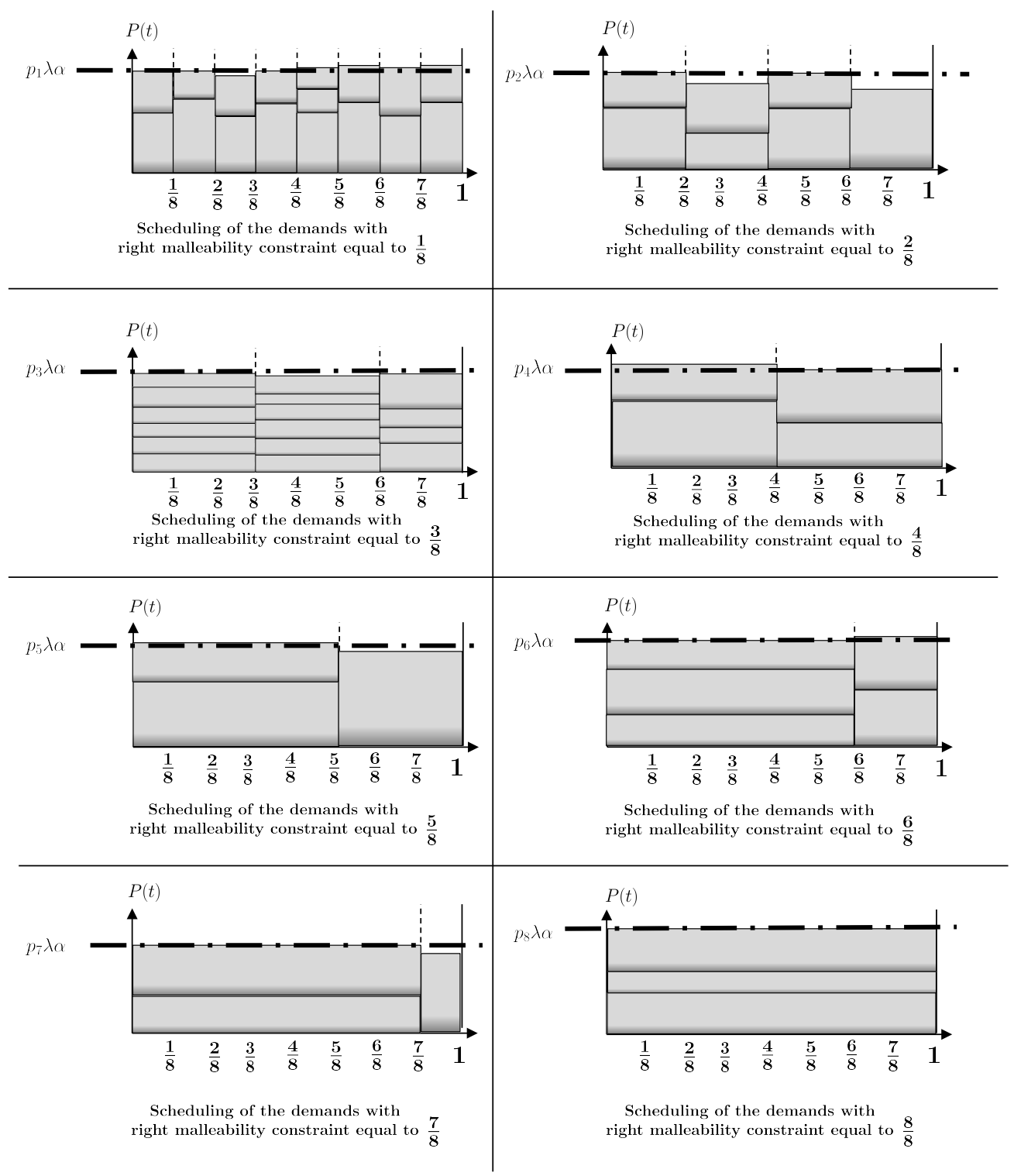

Figure 4.3: Applying the covering policy $\mathbf{v}^{*}$ for scheduling the demands with different right malleability constraints $r_{i}$. Each sub-figure illustrates the scheduling of demands with their right malleability constraints equal to $\frac{k}{8}$, where $k=1, \cdots, d$, and $d=8$. 
represent the total power consumption of demands in $Q_{k, j}^{n}$.

Lemma 7 For $1 \leq k \leq d, 1 \leq j \leq\lceil d / k\rceil$

$$
P_{k, j}^{n} \rightarrow p_{k} \lambda \alpha \quad \text { a.s. } n \rightarrow \infty
$$

Proof.According to the definition of the covering policy $\mathbf{v}^{*}$ (Algorithm 2), for $1 \leq$ $j \leq\lfloor d / k\rfloor:$

$$
\begin{aligned}
& \operatorname{Prob}\left(i \in Q_{k, j}^{n}\right) \\
& =p_{k}\left(\frac{m}{k}\left(1-\frac{k}{d}\right) \frac{1}{\left\lfloor\frac{d}{k}\right\rfloor}+\left(1-\frac{m}{k}\right) \frac{1}{\left\lfloor\frac{d}{k}\right\rfloor}\right) \\
& =p_{k} \frac{1}{\left\lfloor\frac{d}{k}\right\rfloor}\left(1-\frac{m}{d}\right)=p_{k} \frac{k}{d},
\end{aligned}
$$

using $d=k\lfloor d / k\rfloor+m$. The factor $m / k$ appears in (4.24) as a result of Assumption 2 in Subsection 4.2.1). Similarly, if $m \neq 0$, we have

$$
\operatorname{Prob}\left(i \in Q_{k,\left\lceil\frac{d}{k}\right\rceil}^{n}\right)=p_{k} \frac{m}{k} \frac{k}{d}=p_{k} \frac{m}{d}
$$

From the Law of Large Numbers,

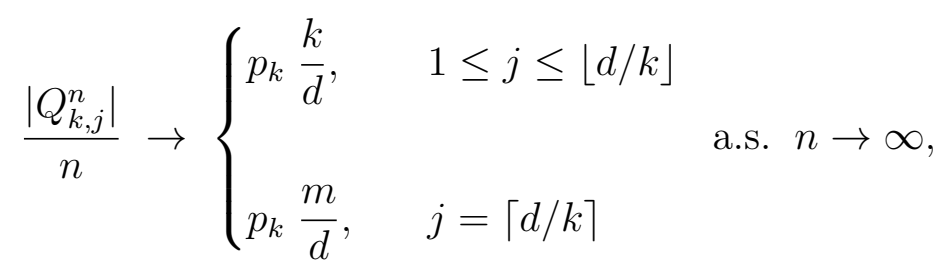

According to the description of Algorithm 2,

$$
s_{i}= \begin{cases}k / d, & i \in Q_{k, j}^{n}, 1 \leq j \leq\lfloor d / k\rfloor \\ m / d, & i \in Q_{k,\lceil d / k\rceil}^{n}\end{cases}
$$


Combining (4.26) - (4.27), Assumptions 1 and 2 (Section 4.2.1), we have:

$$
\begin{aligned}
P_{k, j}^{n} & \rightarrow\left(\frac{\left|Q_{k, j}^{n}\right|}{n}\right)\left(\frac{n}{q_{n}}\right)\left(\frac{1}{\left|Q_{k, j}^{n}\right|} \sum_{i \in Q_{k, j}^{n}} \frac{\xi_{i}}{s_{i}}\right) \\
& \rightarrow p_{k} \lambda \alpha \quad \text { a.s. } n \rightarrow \infty,
\end{aligned}
$$

yielding Lemma 7.

Now introduce $Q_{k}^{n} \triangleq \bigcup_{1 \leq j \leq\lceil d / k\rceil} Q_{k, j}^{n}$ and let $P_{k}^{n}(t)$ be a total power demand over a group $Q_{k}^{n}$ :

$$
P_{k}^{n}(t)=\sum_{i \in Q_{k}^{n}}\left(\frac{A_{i}}{s_{i}} \cdot 1_{\left\{\tau_{i} \leq t \leq \tau_{i}+s_{i}\right\}}\right), 0 \leq t \leq 1
$$

Then for the total cost function (4.9),

$$
P^{\mathbf{v}^{*}, n}(t)=\sum_{k} P_{k}^{n}(t)
$$

According to Algorithm 2, each $P_{k}^{n}(t)$ is piecewise constant, with $\lceil d / k\rceil$ possible values (4.22):

$$
P_{k}^{n}(t)=\left\{\begin{array}{l}
P_{k, j}^{n}, \quad(j-1) \frac{k}{d} \leq t<j \frac{k}{d}, \quad 1 \leq j \leq\lfloor d / k\rfloor \\
P_{k,\lceil d / k\rceil}^{n}, \quad 1-\frac{m}{d} \leq t \leq 1, \quad m \neq 0 .
\end{array}\right.
$$

Applying Lemma 7 to (4.30)-(4.31), yields (4.20), thus concluding Theorem 2.

Theorem 2 implies that the asymptotic costs of applying the covering policy $\mathbf{v}^{*}$ (Algorithm 2) converge a.s. to $L_{P}^{n}$ and $L_{C}^{n}$ (Equation (4.12)), which are the lower bounds for $P_{o p t}^{n}$ and $C_{o p t}^{n}$ (Equations (4.10) and (4.11)) respectively. It means that the covering policy $\mathbf{v}^{*}$ is asymptotically optimal with respect to both cost criteria for maximum power (Equation (4.10)) and the total convex cost of power consumption (Equation (4.11)). 


\subsubsection{Generalization of the "uniformity" assumption}

We have shown the asymptotic optimality of the covering policy $\mathbf{v}^{*}$ under the assumption that given the value of the (arbitrarily distributed) right constraint $r=k / d$, the left constraint $\ell$ is distributed uniformly on $\{1 / d, \ldots, k / d\}$ (Equation (4.8)). This uniformity requirement can be further relaxed, allowing any distribution that satisfies:

$$
\operatorname{Prob}\left(\ell \leq \frac{m}{d} \mid r=\frac{k}{d}\right) \geq \frac{m}{d}, \quad m \triangleq \bmod (d, k)
$$

(since $m<d$, the above inequality clearly includes (4.8)). The corresponding scheduling algorithm will be very similar to Algorithm 2, except for lines 6 and 9, where one should substitute probabilities $k / d$ and $(1-k / d)$ with new proper values. The asymptotic optimality (4.18), (4.19) of a modified algorithm can be carried out in an almost identical way. Details are omitted due to space restriction.

\subsubsection{Fixed Malleability Constraints}

In Chapter 3, we studied the problem of scheduling electrical demands with fixed malleability constraints. There, our assumption was: $\left(\ell_{i}, r_{i}\right) \equiv(\ell, r)$, with a pair $(\ell, r)$ fixed. So for fixed malleability constraints, the relation (4.2) now reads as

$$
\ell \leq s_{i} \leq r, \quad i \in\{1, . ., n\} .
$$

Since the constraints are fixed, the grid $\Omega_{d}$ (Section 4.2.1), is replaced by its continuous counterpart $\Omega$ :

$$
\Omega=\{(\ell, r): 0<\ell \leq r \leq 1\} .
$$

In Chapter 3, for all possible values of $(\ell, r)$, we showed that how to find an asymptotically optimal policy minimizing the power peak. Now in this Chapter, we are going to show how to apply the asymptotic analyses presented in this chapter, for electrical demands with fixed malleability constraints. In particular, we will find the cases, where we could find a policy minimizing the total convex cost, or more precisely, a scheduling policy whose asymptotic performance is the same as the covering policy $\mathbf{v}^{*}$ from Section 4.1 - i.e. it satisfies the relation (4.20), (which leads to Theorem 2 (Equations (4.18) and (4.19))). 


\subsubsection{Full coverage of $[0,1]$ : Characterization}

To derive an asymptotic optimal policy for scheduling demands with fixed constraints, we need to find out how to maximally cover the time interval $[0,1]$ (with no overlapping). To do so, we use some of the definitions and results presented in the previous chapter.

In Chapter 3, we called a real number $w>0$ as coverable, if it can be represented as an integer combination of numbers from the interval $[\ell, r]$. That is, if there exists an integer number $q$ and a set of positive real values: $s_{1}, \ldots, s_{q} \in[\ell, r]$ such that $\sum_{i=1}^{q} s_{i}=w$. Then we showed that, a value $w$ is coverable if and only if:

$$
\lceil w / r\rceil \leq w / \ell
$$

It implies that the point $w=1$ is coverable if and only if:

$$
\lceil 1 / r\rceil \leq 1 / \ell
$$

Then, we defined a coverable region $\mathcal{G}$ to be the set of all pairs $(\ell, r)$ satisfying (4.36) (see Fig. 3.2):

$$
\mathcal{G}=\{(\ell, r), \ell \leq r \text { and } 1 \text { is coverable }\} .
$$

Intuitively, for $(\ell, r) \in \mathcal{G}$, we are able to divide the entire interval $[0,1]$ into $K_{0}=$ $\lceil 1 / r\rceil$ non-overlapping time slots with equal length $S_{0}=1 / K_{0}$. In this case we have $1 / r \leq\lceil 1 / r\rceil \leq 1 / \ell$, as well as $\ell \leq S_{0} \leq r$ which follows due to (4.36). Consequently, we will set the service time of all the demands as $S_{0}$, i.e. $s_{i}=S_{0}, i=1, \ldots, n$. The next theorem summarizes these points.

Theorem 3 Let Assumption 1 hold and let $(\ell, r) \in \mathcal{G}$. The following covering policy $\mathbf{v}^{*}$ is asymptotically optimal, in the sense of (4.18) - (4.19).

Proof of Theorem 3. Decompose the set of all (scheduled by Algorithm 3) demands into a union of disjoints $Q_{j}^{n}, j=1, \cdots, K_{0}=\lceil 1 / r\rceil$ according to

$$
Q_{j}^{n}=\left\{i \in\{1, . ., n\} \quad: \quad \tau_{i}=(j-1) S_{0}\right\}
$$

The scheduling of Algorithm 3 guarantees that the power intensity function $P^{n}(t)$ 


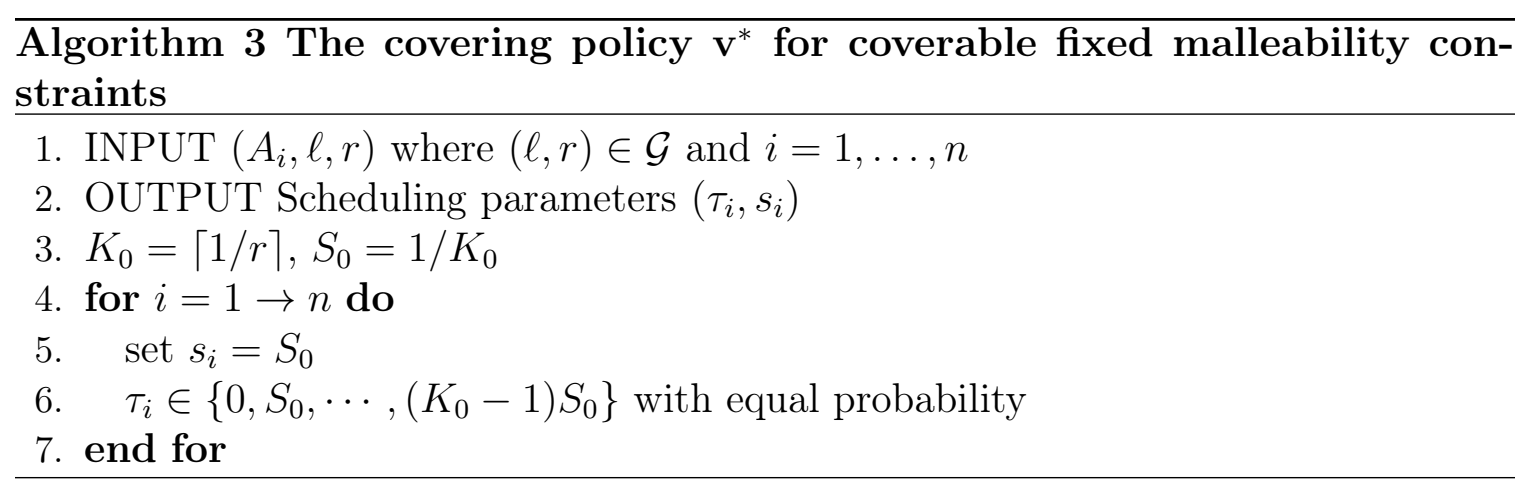

from (4.9) is piecewise constant, with $K_{0}=\lceil 1 / r\rceil$ possible values. Therefore, for each $1 \leq j \leq K_{0}$

$$
P^{n}(t)=P_{j}^{n}=\sum_{i \in Q_{j}^{n}} \frac{A_{i}}{S_{0}}, \quad(j-1) S_{0} \leq t<j S_{0}
$$

For a proof, it would be enough to show that $P^{n}$ satisfies (4.20). Indeed, for $j=$ $1, \cdots, K_{0}=\lceil 1 / r\rceil$, due to Line 6 of the algorithm,

$$
\operatorname{Prob}\left(i \in Q_{j}^{n}\right)=\frac{1}{\lceil 1 / r\rceil}=\frac{1}{K_{0}}=S_{0} .
$$

Therefore, using the Law of Large Numbers,

$$
\frac{\left|Q_{j}^{n}\right|}{n} \rightarrow S_{0} \quad \text { a.s. } n \rightarrow \infty,
$$

Since $s_{i}=S_{0}$ for all $i \in\{1, \cdots, n\}$, we combine (4.41) with the assumptions of the theorem to get

$$
\begin{aligned}
P_{j}^{n} & \rightarrow\left(\frac{\left|Q_{j}^{n}\right|}{n}\right)\left(\frac{n}{q_{n}}\right)\left(\frac{1}{\left|Q_{j}^{n}\right|} \sum_{i \in Q_{j}^{n}} \frac{\xi_{i}}{S_{0}}\right) \\
& \rightarrow \lambda \alpha \quad \text { a.s. } n \rightarrow \infty,
\end{aligned}
$$

concluding the proof.

In Subsection 4.2.5, we explained that Inequality (4.32) is a "sufficient" condition on the distribution of malleability constraint pairs $\left(\ell_{i}, r_{i}\right)$ to cover the entire time interval $[0,1]$ and consequently use Covering Policy $\mathbf{v}^{*}$. Based on that discussion, we only need to become sure that there is statically enough number of demands to be 
put in the last time slot for each value of the right-malleability constraint. For fixed malleability constraints, this implies that the value of $\ell$ should be less than the size of the last time slot. So, we are sure that we are able to put any of the demands in the last time slot of size $m \triangleq \bmod (1, r)=1-r\left\lfloor\frac{1}{r}\right\rfloor$, if necessary. Therefore, for fixed malleability constraints the equivalent condition to Inequality (4.32) is as follows:

$$
\ell \leq 1-r\left\lfloor\frac{1}{r}\right\rfloor
$$

The same as Inequality (4.32) for stochastic malleability constraints, Inequality (4.43) is a "sufficient" condition for applying the covering policy for fixed malleability constraints. However, Inequality (4.36) is a "necessary and sufficient" condition for fix malleability constraint pair $(\ell, r)$ to cover the entire time interval $[0,1]$.

Remark 1 The "necessary and sufficient" condition in Inequality (4.36) encompasses the "sufficient" condition in Inequality (4.43), that is:

$$
\ell \leq 1-r\left\lfloor\frac{1}{r}\right\rfloor \quad \Longrightarrow \quad\left\lceil\frac{1}{r}\right\rceil \leq \frac{1}{\ell}
$$

Proof. Assume $(\ell, r) \notin \mathcal{G}$. Since $\ell \leq r$, we have: $\left\lfloor\frac{1}{r}\right\rfloor=\left\lfloor\frac{1}{\ell}\right\rfloor$, which results in the following inequality:

$$
\ell>1-\ell\left\lfloor\frac{1}{\ell}\right\rfloor \geq 1-r\left\lfloor\frac{1}{r}\right\rfloor
$$

Comparing the above result with Inequality (4.43), proves Remark 1 (Statement (4.44)). Note that Inequality (4.43) is not equivalent to Inequality (4.36). For example given $(\ell, r)=(0.4,0.9),\left\lceil\frac{1}{r}\right\rceil \leq \frac{1}{\ell}$, but $\ell>1-r\left\lfloor\frac{1}{r}\right\rfloor$.

Remark 1 implies that if malleability constraint pair $(\ell, r)$ does not fit into Condition (4.36), it does not fit into Condition (4.43) as well (not the other way around). On the other hand, if a given $(\ell, r)$ satisfies Inequality (4.43), it also satisfies Inequality (4.36). Therefore, for a given $(\ell, r)$, we only need to check whether it satisfies $\left\lceil\frac{1}{r}\right\rceil \leq \frac{1}{\ell}$ or not. Then for all pairs $(\ell, r) \in \mathcal{G}$, we will use Covering policy in Algorithm 3 , which is asymptotically optimal for both maximal power and convex costs. 


\subsubsection{Partial coverage of $[0,1]$ : Optimality of maximum Power}

In the previous subsection, for fixed malleability constraints, we showed that how to use the necessary and sufficient condition in Inequality (4.36) to cover the entire time interval $[0,1]$ and then use the asymptotically optimal scheduling in Algorithm 3. On the other hand, as it is shown in Chapter 3, for the values of $(\ell, r) \notin \mathcal{G}$, we are not able to cover the entire time interval $[0,1]$ and we cannot use Covering Policy $\mathbf{v}^{*}$ minimizing both power peak and total convex cost criteria. However, as discussed in Section 3.2 (Theorem 1), even for the values of $(\ell, r) \notin \mathcal{G}$, we are still able to introduce asymptotically optimal scheduling policy with respect to maximum power cost criteria from (4.10). Here, we are going to use the results of Section 3.2 (Theorem 1) and apply the asymptotic analyses performed in this Chapter, to prove this. Again, we are going to use some definitions and results from Chapter 3.

Assume $w$ is not coverable, that is $\lceil w / r\rceil>w / \ell$, (see Lemma 1). Define its largest coverable point $w^{*}=w^{*}(w)$ as:

$$
w^{*}=\sup \{v: v<w, \text { such that } v \text { is coverable. }\}
$$

Then in Lemma 2, we showed that: If $w$ is not coverable, then $w^{*}(w)=r\lfloor w / r\rfloor$. We denoted by $Z^{*}$, the largest coverable point for $w=1$. Using Lemma 2 , if $(\ell, r) \notin \mathcal{G}$, then $Z^{*}=Z^{*}(1)=r\lfloor 1 / r\rfloor$.

Then, we introduced Lemma 1, stating that: Let $A=\sum_{i=1}^{n} A_{i}$ and $(\ell, r) \notin \mathcal{G}$. Then for the maximum power function from (4.10),

$$
P_{o p t}^{n} \geq \frac{A}{Z^{*}}
$$

Since $Z^{*}<1$, this lemma gives us a tighter lower bound than (4.13). Therefore, when $(\ell, r) \notin \mathcal{G}$, we can schedule all the demands on $\left[0, Z^{*}\right]$ using a very intuitive policy: divide $\left[0, Z^{*}\right]$ into $K_{0}=\lfloor 1 / r\rfloor$ non-overlapping time slots with equal length $r$ and leave the remaining part of the time interval, i.e. $\left[Z^{*}, 1\right]$ uncovered. This is formalized in Algorithm 4.

Theorem 4 Let Assumption 1 hold and let $(\ell, r) \notin \mathcal{G}$. Then:

$$
P_{o p t}^{n} \rightarrow \frac{\lambda \alpha}{Z^{*}}, \quad \text { a.s. } n \rightarrow \infty,
$$




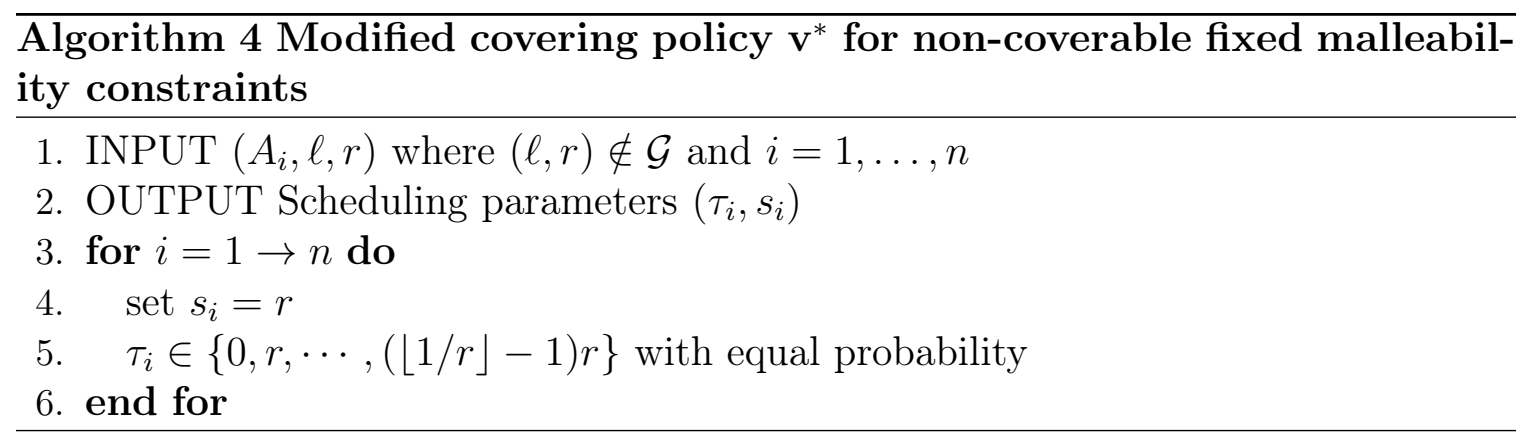

Proof of Theorem 4: decompose the set of all scheduled demands into a unit of disjoint sets $Q_{j}^{n}, j=1, \cdots,\lfloor 1 / r\rfloor$, as follows:

$$
Q_{j}^{n}=\left\{i \in\{1, . ., n\}: \tau_{i}=(j-1) r\right\}
$$

The resulting power intensity function $P^{n}(t)$ from (4.9) is piecewise constant, with $\lfloor 1 / r\rfloor$ possible values. In particular, for $1 \leq j \leq\lfloor 1 / r\rfloor$, we have:

$$
P^{n}(t)=P_{j}^{n}=\sum_{i \in Q_{j}^{n}} \frac{A_{i}}{r}, \quad(j-1) r \leq t<j r .
$$

The result (4.47) will follow once we show that

$$
P_{j}^{n} \rightarrow \frac{\lambda \alpha}{Z^{*}}, \quad \text { a.s. } n \rightarrow \infty
$$

for all $1 \leq j \leq\lfloor 1 / r\rfloor$. Indeed, for such $j$, using Algorithm 4, (Line 5), we have:

$$
\operatorname{Prob}\left(i \in Q_{j}^{n}\right)=\frac{1}{\lfloor 1 / r\rfloor} .
$$

After that, using the Law of Large Numbers

$$
\frac{\left|Q_{j}^{n}\right|}{n} \rightarrow \frac{1}{\lfloor 1 / r\rfloor}, \quad \text { a.s. } n \rightarrow \infty
$$


Recall $Z^{*}=r\lfloor 1 / r\rfloor$. Combining (4.52) with Assumption 1,

$$
\begin{aligned}
P_{j}^{n} & \rightarrow\left(\frac{\left|Q_{j}^{n}\right|}{n}\right)\left(\frac{n}{q_{n}}\right)\left(\frac{1}{\left|Q_{j}^{n}\right|} \sum_{i \in Q_{j}^{n}} \frac{\xi_{i}}{r}\right) \\
& \rightarrow \frac{\lambda \alpha}{Z^{*}}, \quad \text { a.s. } n \rightarrow \infty,
\end{aligned}
$$

for all $j$, yielding (4.47).

\subsection{Computational Results for Covering Policy}

Our study is the first one taking into account malleability in the scheduling problem of rectangular-shape electrical demands (Chapter 2). In other studies, considering Strip Packing Problem of rectangular-shape electrical demands, such as [68-71] the following generated data sets are being used: Mumford-Valenzuela [85], Hopper and Turton [58], Burke [86] and especially Pedro and Guillermo Sanchez [87] Benchmarks. Unfortunately, demand malleability has not been addressed in these existing data sets. However, we expect that by the presence of more flexible-rate chargeable devices, even some real data sets would be accessible. In those data sets, the values of energy requirements, as well as malleability constraint will follow some distributions.

In this section, in order to examine our proposed policies, we will generate some data sets with respect to our assumptions in Subsection 4.2.1, which are rewritten in Table 4.1. Then we will illustrate the output of Covering policy for different scenarios discussed so far. In our numerical experiments, we examined covering policy for different values of the number $n$ of demands. Using the notation from Section 4.1, we assume $\lambda=1$ and $q_{n}=n$. Also for energy demand values, we assume that $A_{i} \stackrel{d}{=} \xi / q_{n}$, where $\xi_{i}$ 's are i.i.d Exponential random variables with mean $\alpha=10$. To evaluate the performance of covering policy we consider two cost criteria defined in Section 4.1: "maximum power", $P_{\max }^{\pi}$ (Equation (4.10)) and "Total Convex Cost" of power consumption, $C^{\pi}$ (Equation (4.11)).

First, we assume that malleability constraints $\left(\ell_{i}, r_{i}\right), i=1, \cdots, n$, are i.i.d. random vectors, uniform in $\Omega_{d}$.In addition, to examine the effect of choosing different values of $d$, we examine the Covering policy $\mathbf{v}^{*}$ for different values of $d$. Figures 4.4, 4.5, 4.6 and 4.7 illustrate the outputs of policy $\mathbf{v}^{*}$ in Algorithm 2, for 
Table 4.1: The summary of the Assumptions in Subsection 4.2.1

\begin{tabular}{|c|c|}
\hline Assumption & The Assumption Summary \\
\hline Assumption 1 & $\begin{array}{l}\text { Let }\left\{\xi_{i}, i=1, \ldots, n\right\} \text { be a sequence of i.i.d. random variables } \\
\text { with } \alpha:=E\left(\xi_{i}\right)<\infty \text { and } \operatorname{Var}\left(\xi_{i}\right)<\infty \text {. Then for the } \\
\text { demand size distribution, we assume : } \\
\qquad A_{i} \stackrel{d}{=} \xi_{i} / q_{n}, \\
\text { where }\left\{q_{n}\right\} \text { is an increasing sequence, satisfying: } \\
\qquad n / q_{n} \rightarrow \lambda \\
\text { as } n \rightarrow \infty \text { for some } \lambda \in(0, \infty) .\end{array}$ \\
\hline Assumption 2 & $\begin{array}{l}\text { The random pairs }\left\{\left(\ell_{i}, r_{i}\right)\right\}, i \in\{1, \ldots, n\} \text { are independently } \\
\text { and identically distributed in } \Omega_{d} \text { according to the following } \\
\text { law: } \\
\text { - The value of the right constraint } r_{i} \text { is assumed to have } \\
\text { an arbitrary discrete distribution in }\left\{\frac{1}{d}, \frac{2}{d}, \ldots, 1\right\} \text {, with: } \\
\qquad p_{k}:=\operatorname{Prob}\left(r_{i}=\frac{k}{d}\right) . \\
\text { - given } r_{i}=k / d \text {, the left constraint } \ell_{i} \text { is uniformly dis- } \\
\text { tributed in }\left\{\frac{1}{d}, \ldots, \frac{k}{d}\right\} \text {, that is for } k^{\prime} \leq k \text { : } \\
\qquad \operatorname{Prob}\left(\ell_{i} \leq \frac{k^{\prime}}{d} \mid r_{i}=\frac{k}{d}\right)=\frac{k^{\prime}}{k} .\end{array}$ \\
\hline
\end{tabular}

$n \in\{500,1000,1500, \cdots, 10000\}$, when $d=3, d=9, d=20$ and $d=100$ respectively. By looking into these figures, we can see that for all different values of $d$, both peak power and the total convex costs eventually converges to their optimal values. However, for smaller values of $d$, which is equivalent to some bigger number of demands in each time slot, the convergence happens relatively faster. Moreover, to see the performance of the Covering policy $\mathbf{v}^{*}$ in the presence of bigger numbers of demands, we have done the same practice for $n \in\{500,5500,10500, \cdots, 100000\}$. 


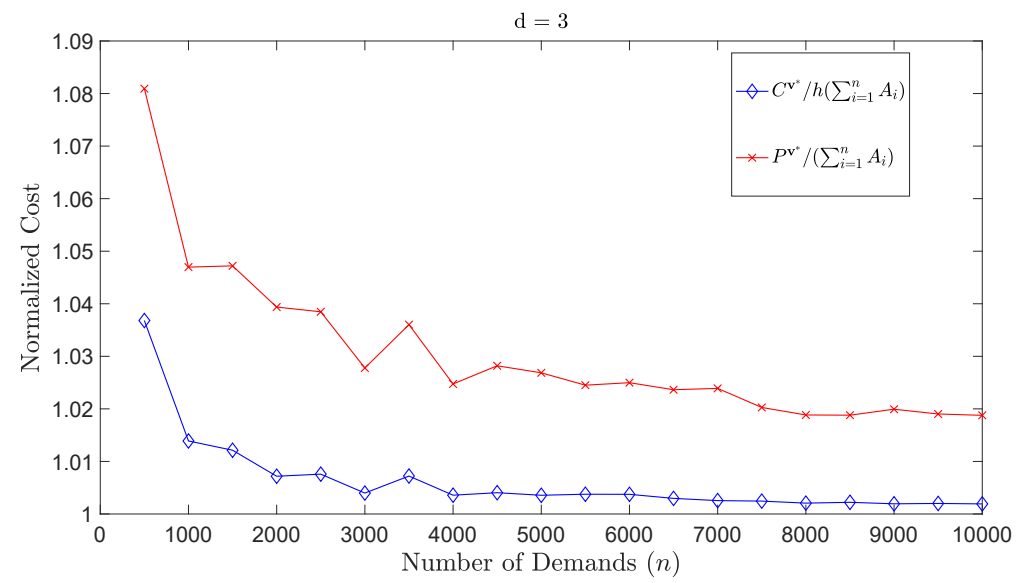

Figure 4.4: Performance of the covering policy $\mathbf{v}^{*}$ in Algorithm 2 with respect to both cost criteria: maximum power and the total convex cost of power consumption, where malleability constraint pairs are i.i.d random vectors in $\Omega_{d}$, with $d=3$ and $n \in\{500,1000,1500, \cdots, 10000\}$.

Figures 4.8, 4.9, 4.10 and 4.11 illustrate the resulted outputs.

In the second scenario, we consider fixed malleability constraints (Section 4.2.6), where $(\ell, r)=\left(\frac{1}{3.8}, \frac{1}{2.32}\right)$. Such constraints satisfy the necessary and sufficient condition in Inequality (4.36), i.e. $(\ell, r) \in \mathcal{G}$. So in this case, we are able to cover the entire interval $[0,1]$ and perform covering policy in Algorithm 3. The outputs of Algorithm 2 are illustrated in Figure 4.12, for different values of $n$. Finally we consider a case where malleability constraint pair $(\ell, r)$ is fixed but does not belong to the coverable region $\mathcal{G}$ (Equation (4.37) and Figure 3.2). In particular, we let $(\ell, r)=\left(\frac{1}{2.8}, \frac{1}{2.32}\right)$. As we can see, $(\ell, r)$ does not satisfy Inequality (4.36). In this case, as we discussed in Subsection 4.2.6.2, even though we are not able to cover the entire interval $[0,1]$, using policy from Algorithm 4, the maximum power cost $P_{\max }$ is still asymptotically optimal. Figure 4.13 illustrates the outputs of policy $\mathbf{v}^{*}$ in Algorithm 4, for different values of $n$. 


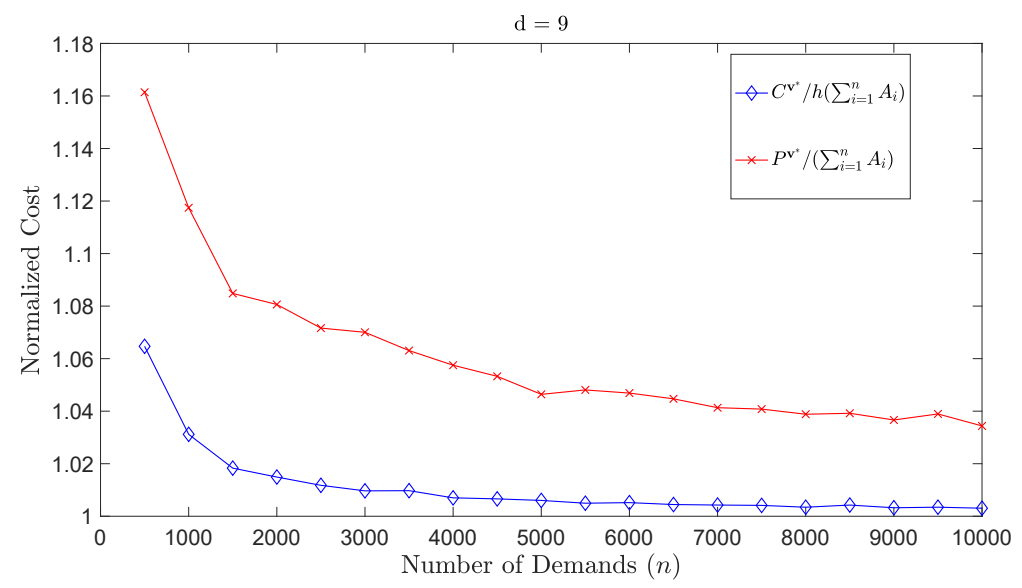

Figure 4.5: Performance of the covering policy $\mathbf{v}^{*}$ in Algorithm 2 with respect to both cost criteria: maximum power and the total convex cost of power consumption, where malleability constraint pairs are i.i.d random vectors in $\Omega_{d}$, with $d=9$ and $n \in\{500,1000,1500, \cdots, 10000\}$.

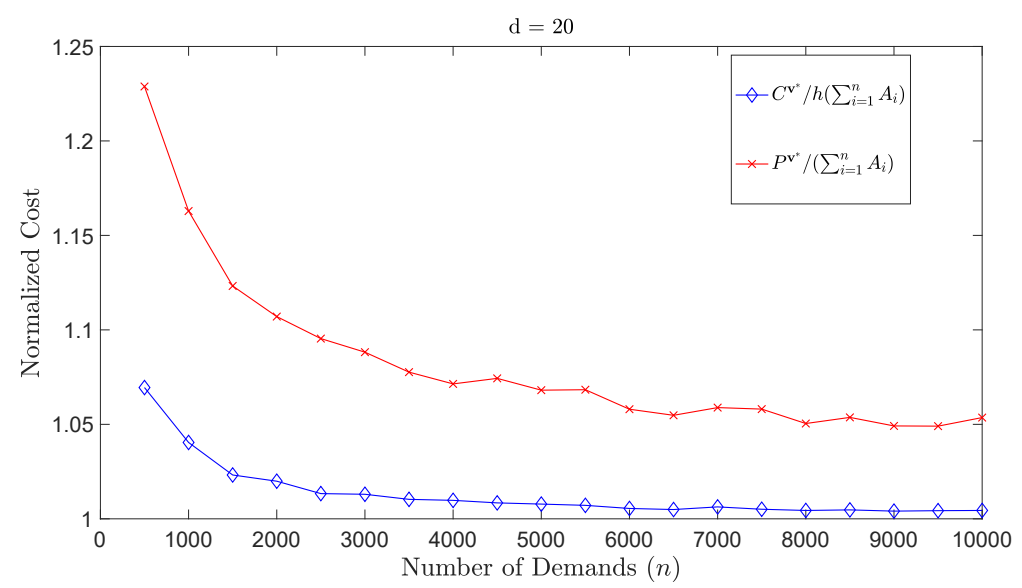

Figure 4.6: Performance of the covering policy $\mathbf{v}^{*}$ in Algorithm 2 with respect to both cost criteria: maximum power and the total convex cost of power consumption, where malleability constraint pairs are i.i.d random vectors in $\Omega_{d}$, with $d=20$ and $n \in\{500,1000,1500, \cdots, 10000\}$. 


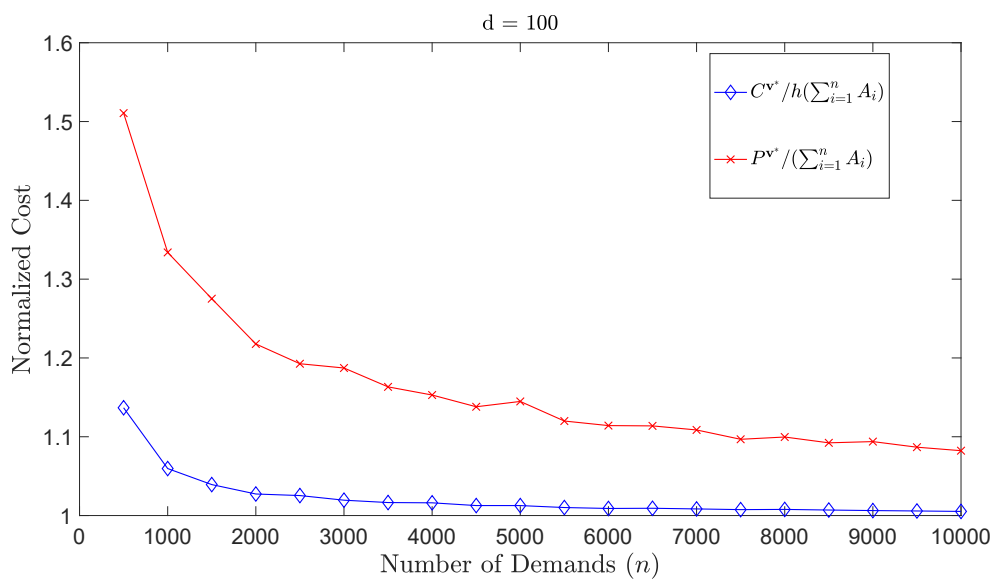

Figure 4.7: Performance of the covering policy $\mathbf{v}^{*}$ in Algorithm 2 with respect to both cost criteria: maximum power and the total convex cost of power consumption, where malleability constraint pairs are i.i.d random vectors in $\Omega_{d}$, with $d=100$ and $n \in\{500,1000,1500, \cdots, 10000\}$.

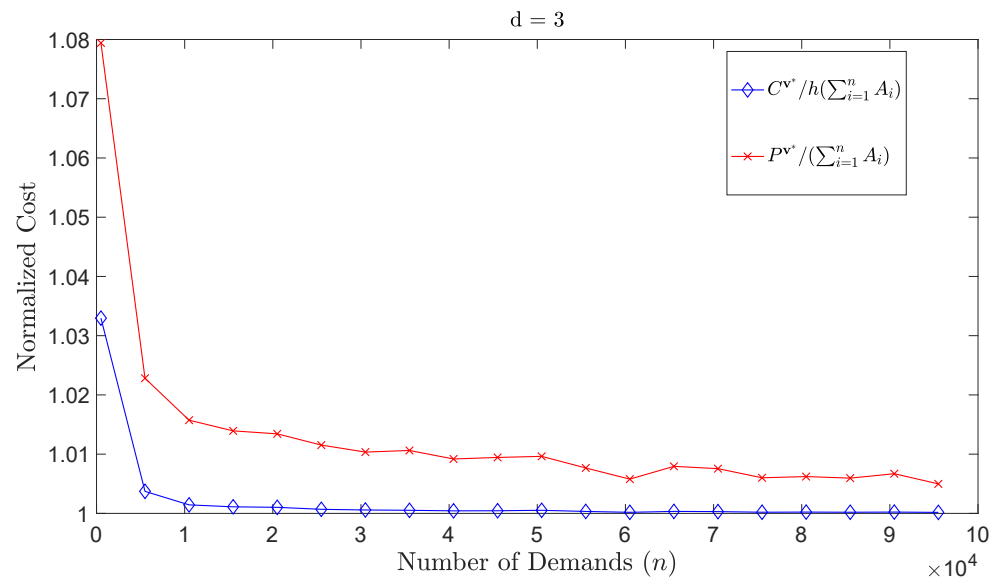

Figure 4.8: Performance of the covering policy $\mathbf{v}^{*}$ in Algorithm 2 with respect to both cost criteria: maximum power and the total convex cost of power consumption, where malleability constraint pairs are i.i.d random vectors in $\Omega_{d}$, , with $d=3$ and $n \in\{500,5500,10500, \cdots, 100000\}$. 


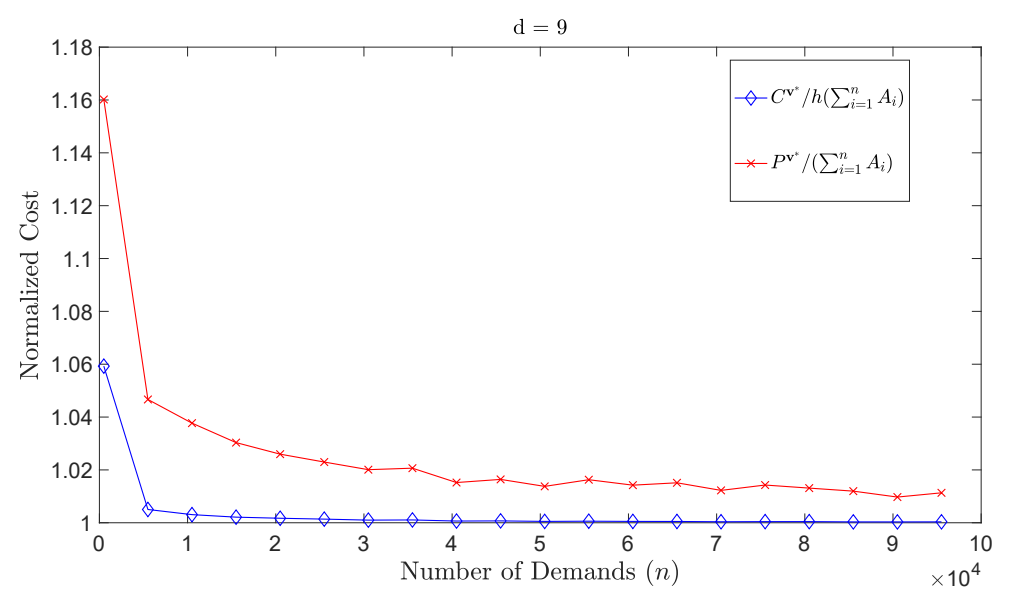

Figure 4.9: Performance of the covering policy $\mathbf{v}^{*}$ in Algorithm 2 with respect to both cost criteria: maximum power and the total convex cost of power consumption, where malleability constraint pairs are i.i.d random vectors in $\Omega_{d}$, with $d=9$ and $n \in\{500,5500,10500, \cdots, 100000\}$.

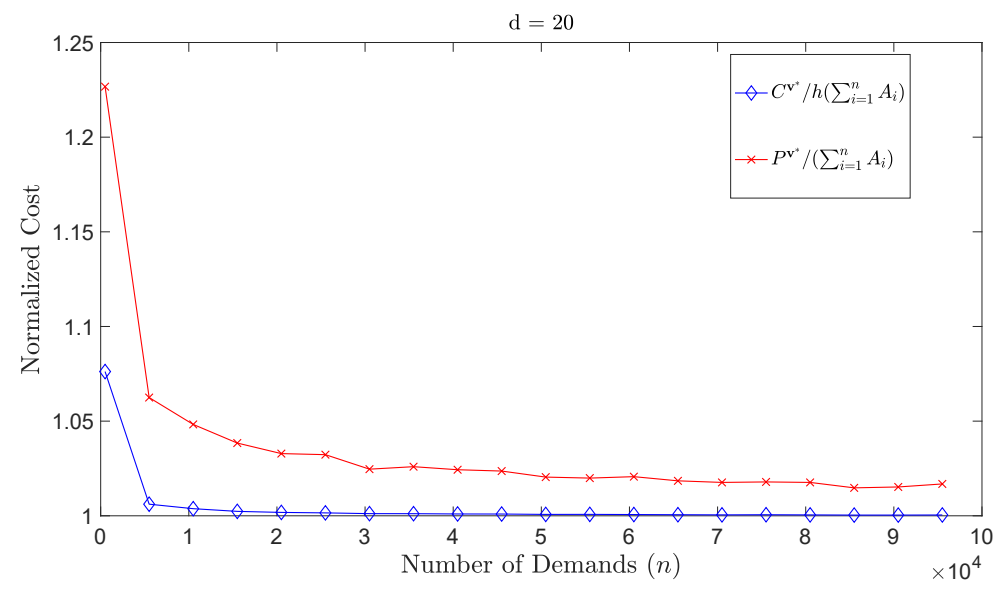

Figure 4.10: Performance of the covering policy $\mathbf{v}^{*}$ in Algorithm 2 with respect to both cost criteria: maximum power and the total convex cost of power consumption, where malleability constraint pairs are i.i.d random vectors in $\Omega_{d}$, with $d=20$ and $n \in\{500,5500,10500, \cdots, 100000\}$. 


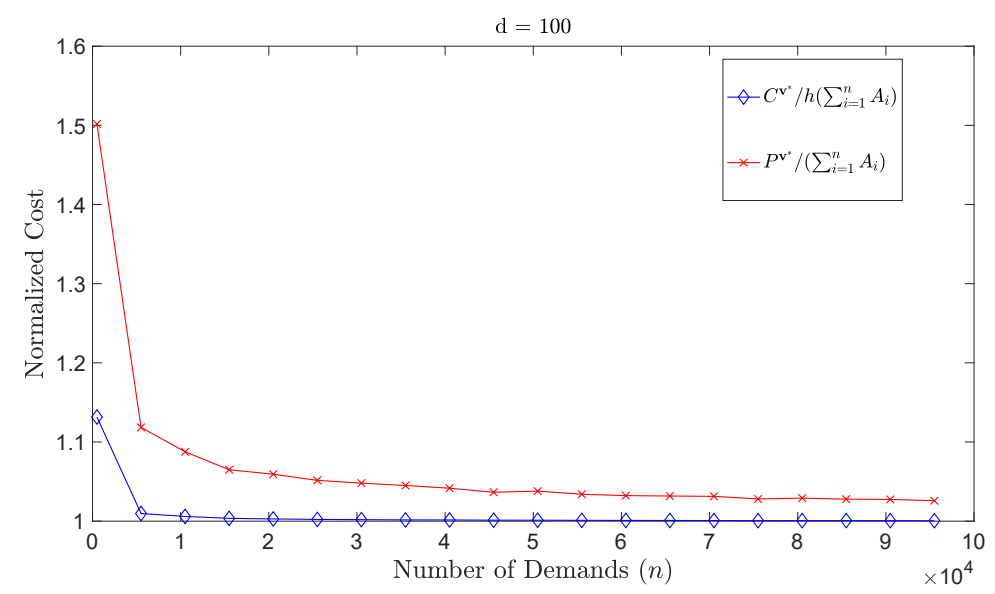

Figure 4.11: Performance of the covering policy $\mathbf{v}^{*}$ in Algorithm 2 with respect to both cost criteria: maximum power and the total convex cost of power consumption, where malleability constraint pairs are i.i.d random vectors in $\Omega_{d}$, with $d=100$ and $n \in\{500,5500,10500, \cdots, 100000\}$.

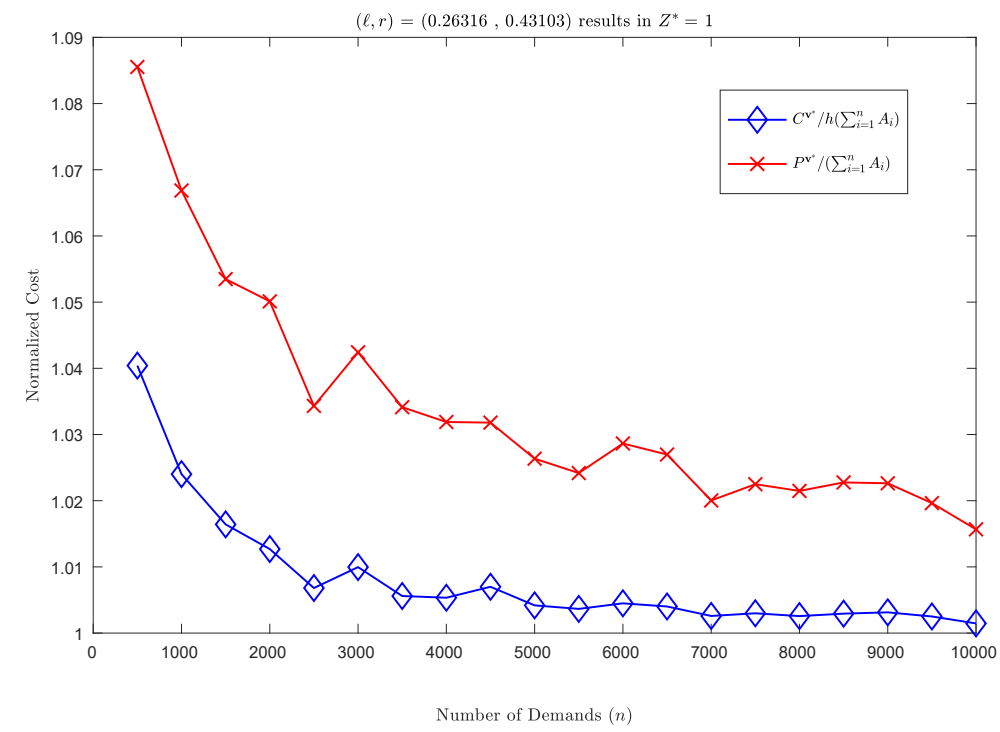

Figure 4.12: Performance of the covering policy $\mathbf{v}^{*}$ in Algorithm 3 with respect to both cost criteria: maximum power and the total convex cost of power consumption, where malleability constraint pair is fixed and is in the coverable region $\mathcal{G}$, that is $Z^{*}=1$. 


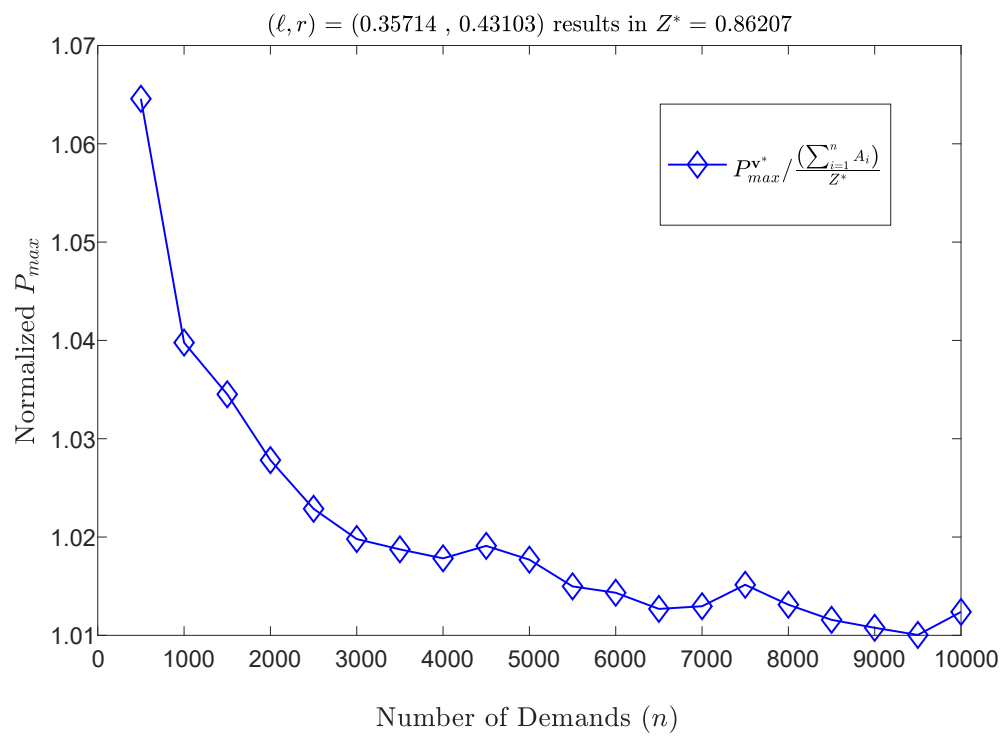

Figure 4.13: Performance of the covering policy $\mathbf{v}^{*}$ in Algorithm 4 with respect to Peak criteria, where malleability constraint pair is fixed, but is not in the coverable region $\mathcal{G}$, that is $Z^{*}<1$.

As it is illustrated in all the figures, the performance of the covering policy gets better as the number of demands increases, which is consistent with our asymptotic analysis.

\subsection{Sequential Application of Covering Policy}

So far, we studied the problem of optimal scheduling of malleable power demands where all demands are available before scheduling starts. In this section, we will show how to deploy the proposed policies when demands are arriving randomly during an arbitrary length time horizon. We assume that the finite scheduling horizon is $[0, T]$, with integer $1<T<\infty$. This time horizon is divided into intervals of unit length. All demands arriving in an interval have to be scheduled in the next interval (see Figure 4.14). Demands being scheduled in the first interval are the ones arriving before scheduling starts. The resulted scheme is considered as a version of the moving horizon control paradigm.

For each $j, j=1, \cdots, T$, the number of demands to be scheduled in each time 


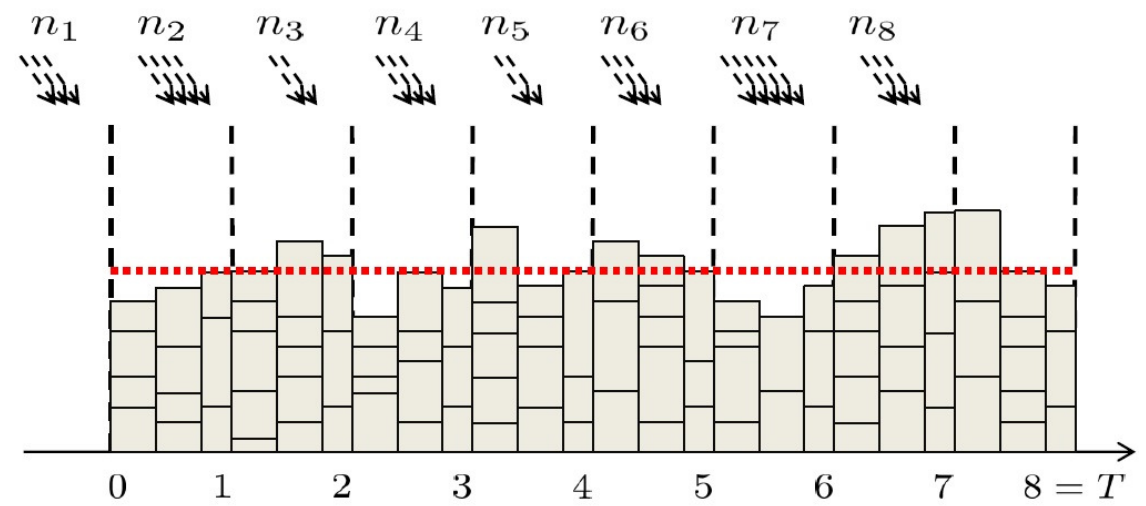

Figure 4.14: Applying the covering policy for scheduling demands in time interval $[0, T]$. Demands are arriving in different time intervals of unit length and have be to be scheduled in the next time interval. The dotted horizontal line shows the average power consumption.

interval $[j-1, j]$ is given by a random variable $n_{j}$, where $n_{j}$ 's are i.i.d Poisson random variables with average $\bar{n}$. We seek a scheduling policy $\pi=\left\{\left(\tau_{i}, s_{i}\right), i=1, . ., \sum_{j=1}^{T} n_{j}\right\}$, such that:

- The demand $A_{i}$, which has arrived in time interval $[j-2, j-1], j=2, \cdots, T$ or before $t=0$ for $j=1$, is being supplied with a constant power $A_{i} / s_{i}$ on $\left[\tau_{i}, \tau_{i}+s_{i}\right] \subset[j-1, j]:$

- Its duration $s_{i}$ satisfies the malleability constraint

$$
\ell_{i} \leq s_{i} \leq r_{i}, \quad i \geq 1
$$

with $\left\{\left(\ell_{i}, r_{i}\right)\right\}_{i \geq 1}$ random, satisfying Assumption 2 .

Asymptotic regime: We assume $\bar{n} \rightarrow \infty$; that is, as before, we assume the number of power demands to be large. Following (and somewhat simplifying) Assumption 1 of Section 4.2.1, we assume that energy demands, $A_{i}$ 's, $i=1,2, \cdots$ are given as:

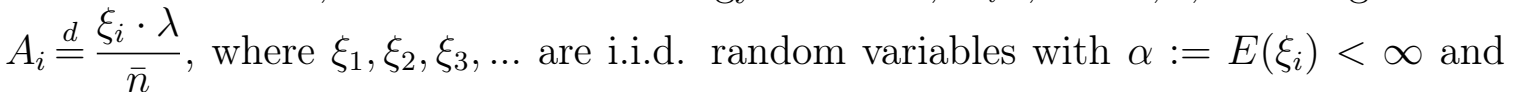
$\operatorname{Var}\left(\xi_{i}\right)<\infty$; that is the size of each demand is inversely proportional to $\bar{n}$, the average number of demands in each time interval. Assumption 2 of Section 4.2.1 remains the same.

Let $N=\sum_{j=1}^{T} n_{j}$ be a random variable describing the total number of demands. Similarly to (4.9), for a policy $\pi$, the total power consumption of the system at time 
$t \in[0, T]$ is:

$$
P^{\pi}(t)=\sum_{i=1}^{N}\left(\frac{A_{i}}{s_{i}} \cdot 1_{\left\{\tau_{i} \leq t \leq \tau_{i}+s_{i}\right\}}\right)
$$

Similarly to Equation (4.9), Equation (4.55) (as well as Equations (4.56) - (4.60)) depends on $\bar{n}$, but for the clarity of presentation, the superscript $\bar{n}$ is omitted. Analogously to (4.10) and (4.11), we seek the optimal values of two cost functions: the maximum power and total convex cost of power consumption:

$$
\begin{aligned}
& P_{o p t}=\inf _{\pi \in \Pi} P_{\max }^{\pi}=\inf _{\pi \in \Pi}\left(\max _{t \in[0, T]}\left\{P^{\pi}(t)\right\}\right), \\
& C_{\text {opt }}=\inf _{\pi \in \Pi} C^{\pi}=\inf _{\pi \in \Pi}\left(\int_{t=0}^{T} h\left(P^{\pi}(t)\right) d t\right),
\end{aligned}
$$

which have the following lower bounds (see (4.13) - (4.15)):

$$
\widetilde{L}_{P}=\frac{1}{T} \sum_{i=1}^{N} A_{i}, \quad \widetilde{L}_{C}=T \cdot h\left(\frac{1}{T} \sum_{i=1}^{N} A_{i}\right),
$$

where $L_{C}$ is obtained using Jensen's inequality as follows:

$$
\int_{t=0}^{T} h\left(P^{\pi}(t)\right) d t \geq T \cdot h\left(\frac{1}{T} \int_{t=0}^{T} P^{\pi}(t) d t\right)
$$

Using the Asymptotic regime introduced in this Section, we rewrite $\widetilde{L}_{P}$ as follows:

$$
\widetilde{L}_{P}=\frac{1}{T} \sum_{i=1}^{N} \frac{\xi_{i} \cdot \lambda}{\bar{n}}=\left(\frac{N}{\bar{n} T}\right) \cdot \lambda \cdot\left(\frac{1}{N} \sum_{i=1}^{N} \xi_{i}\right) .
$$

With our assumptions on $n_{j}$ 's, $N=\sum_{j=1}^{T} n_{j}$ is a Poisson random variable with mean $\bar{n} T$. So as $\bar{n} \rightarrow \infty,\left(\frac{N}{\bar{n} T}\right) \rightarrow 1$. Eventually using the Law of Large Numbers results in:

$$
\left\{\begin{array}{l}
\widetilde{L}_{P} \rightarrow \lambda \alpha \\
\widetilde{L}_{C} \rightarrow T \cdot h(\lambda \alpha) \quad \text { a.s. } \bar{n} \rightarrow \infty,
\end{array}\right.
$$

So, essentially, we can use the covering policy $\mathbf{v}^{*}$ to schedule demands in each unit interval, expecting to achieve asymptotically optimal performance - that is, the costs 
(4.56)-(4.57) are converging to $(\lambda \alpha)$ and $T \cdot h(\lambda \alpha)$ respectively.

We conclude the Section with the following numerical experiment. Let $T=8$, and assume that the number of demands in different time intervals, $n_{j}$ 's, are i.i.d Poisson random variable with mean $\bar{n}$. We consider $\xi_{i}$ 's as i.i.d Exponential random variables with $\alpha=10$. We let $\lambda=1$. So for energy demands we will have: $A_{i} \stackrel{d}{=} \xi_{i} / \bar{n}$. We also assume that malleability constraints $\left(\ell_{i}, r_{i}\right), i \geq 1$, are random vectors independently and identically distributed in $\Omega_{d}$, with $d=9$.

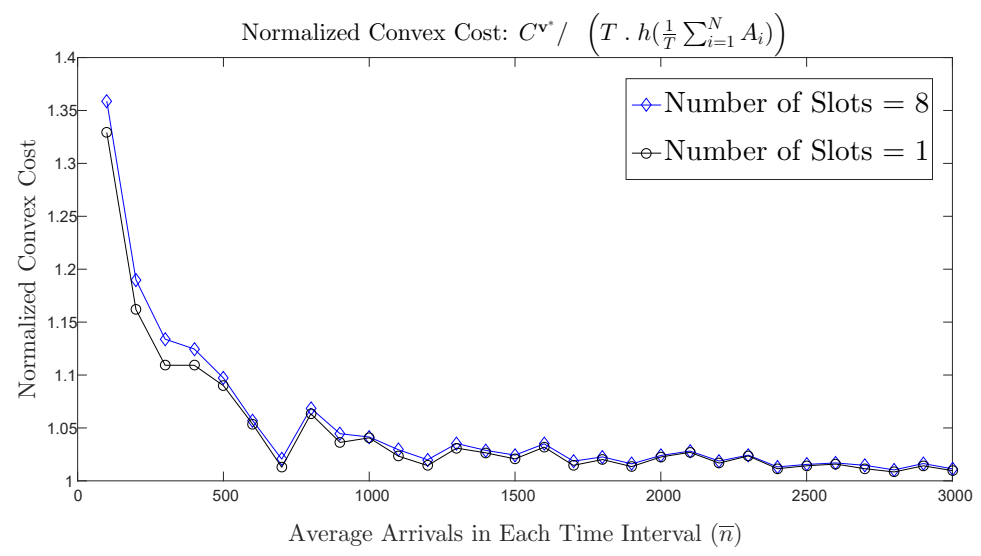

Figure 4.15: Long-Term performance of the covering policy $\mathbf{v}^{*}$ with respect to total operational convex cost. Malleability constraint pairs are i.i.d uniform random vectors in $\Omega_{d}$. The whole scheduling time interval is $[0,8]$.

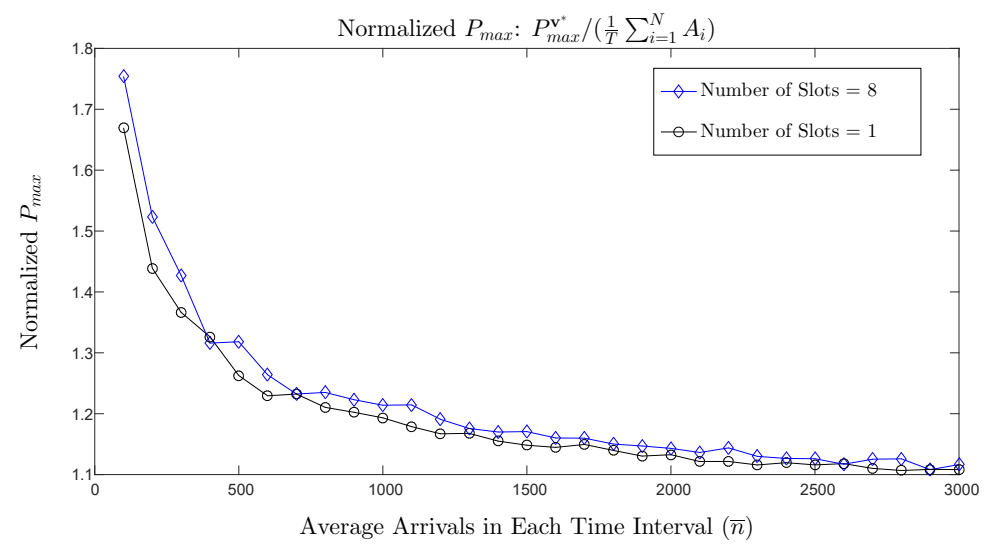

Figure 4.16: Long-Term performance of the covering policy $\mathbf{v}^{*}$ with respect to Peak criteria. Malleability constraint pairs are i.i.d uniform random vectors in $\Omega_{d}$. The whole scheduling time interval is $[0,8]$. 
Figures 4.15 and 4.16 illustrate the outputs of the policy $\mathbf{v}^{*}$ in Algorithm 2, for different values of $\bar{n}$. In these figures, we compared the performance of the policy in two different scenarios: The first scenario is the same as what we discussed in this Section. We apply the covering policy to schedule the demands, which arrived in the previous time slot. For the second scenario, we use the same setting we had in Section 4.3. That is, we assume that all the demands are already available at the time 0 and can be scheduled in the entire $[0, T]$ ( as long as $0 \leq \tau_{i}<\tau_{i}+s_{i} \leq T$ ). In this case, we can simply normalize the "time axes" values, with respect to $T=8$ and then use the policy in Algorithm 2. Similarly to Section 4.3, we used $h(x)=x^{4}$ for a convex cost. As the parameter $\bar{n}$ increases, Figure 4.15 demonstrate that the performance of the first scenario approaches that of the second scenario. This behaviour is consistent with the asymptotic analysis.

\subsection{Summary}

We considered a problem of scheduling "flexible" electrical power demands in the smart grid. Our model consists of a large number of stochastic energy requirements, needed to be scheduled in a finite time interval $[0,1]$. For each demand, we defined the left and right malleability constraints $\ell_{i}$ and $r_{i}$ on the length of the time period, during which the demand has to be supplied without interruption. The malleability constraint pairs $\left\{\left(\ell_{i}, r_{i}\right)\right\}, i \in\{1, . ., n\}$ are assumed to be random vectors, independently and identically distributed based on some distribution. Especially the right constraint $r_{i}$ was assumed to have any arbitrary distribution. Having the objective of minimizing the maximum power and the total convex cost of power consumption in a smart grid, we proposed a linear-time scheduling policy and showed its asymptotic optimality with respect to both cost functions. At the end, we showed how to use the proposed scheduling policies for long-term scenarios. We verified our analyses by presenting the outcomes of some computational experiments.

As an extension to this study, we may consider the optimization problem in cases where an electrical grid carries flexible and non-flexible demands, i.e. some demands are not malleable and/or cannot tolerate delay in receiving their service. Furthermore, we may take into account the pre-emptive scheduling, where a scheduler is able to pause/stop the service of a demand. As another important extension, we may consider scenarios where the objective is real-time serving the arriving demands with different 
stochastic characteristics such as energy requirements, malleability constraints and the acceptable delay to receive the service. 


\section{Chapter 5}

\section{Optimal Controlling of Charging Stations for Electrical Vehicles under Real Time Pricing}

In this chapter, we address the problem of controlling a charging station of Electric Vehicles (EVs). We first introduce a model for the charging station, in which EVs are charged either directly from the electrical grid or from a local storage unit. We assume that the price of the electrical power unit is determined by a Real-Time Pricing scheme, in which, the price of the electrical power is continuously updated with respect to the state of the grid at each time instance. Our goal is to find a control policy minimizing the utilization cost of the charging station. To do so, we model the charging station as a continuous time Markov Decision Process. The control actions to be determined by the charging station operator are as follows: the probability of blocking new arrivals, the rate of charging the local storage unit and the proportion of cars being served by the local storage unit. By using dynamic programming, we prove the existence of the optimal policy minimizing a discounted cost over an infinite time horizon. We also show that it is stationary and bang-bang, i.e. the admissible action set in the optimal policy assumes only the extreme values in the action set. Eventually, we will define the infinite horizon average cost and then we will show how to find the average cost optimal policy from a discounted cost optimal policy.

\subsection{Charging Station Model: Overall View}

In this chapter, we are proposing a model for a charging station of EVs. This model is an extension of the ones presented in [72,79,80]. In particular, in [72, 79, 80], authors simply use some heuristic policies, without trying to find the optimal policies. Here, 


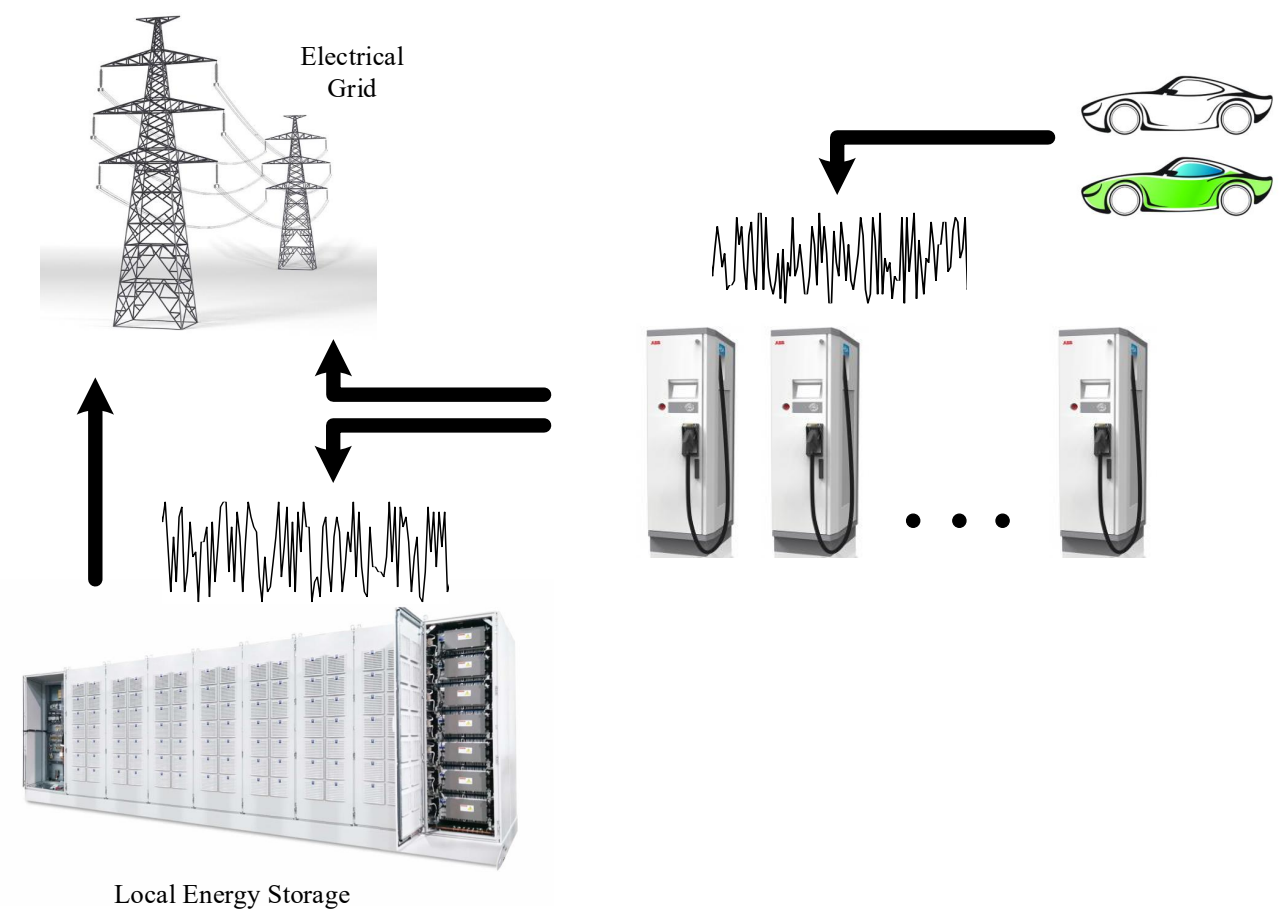

Figure 5.1: The overall view of the proposed charging station

we extend the problem, by precisely define the control actions. Then we will show how to derive the optimal policy minimizing the long-term cost of deploying the charging station. The overall picture of the proposed model will be presented here, then the detailed mathematical model will be discussed in the next Section.

One of the main objectives of the proposed model is to isolate the peak demands from the electrical grid. As we mentioned earlier, charging a large number of EVs at the same time, will push the peak demand in the electrical grid. To address this concern, the amount of the electrical power drawn from the grid is limited to a predetermined value. This value is usually determined as part of a contract between station and grid operators. Based on such contracts, charging station operators agree to a limited power level in return for lower prices. On the other hand, the grid is benefited by having by better estimate of its demands. Consequently having these kinds of contracts will decrease the cost of utilizing the charging stations and increase the overall efficiency of the grid $[88,89]$.

To be able to better serve the stochastic charging demands, a local energy storage 
is deployed in the station. It can be used to meet the excessive demands during rush hours and consequently smooth the total stochastic energy requirement profile of the station. On the other hand, if possible, any unused power from the grid will be used to charge this local storage unit.

The total available power from the grid is descritized and split into $S$ equal slots. It means that using the total available power from the grid can be used to charge up to $S$ EVs at the same time. Similarly, a local storage unit can accommodate up to $R$ EVs, if it is fully charged. Figure 5.1, shows an overall view of the introduced charging station.

The total available power from the grid and from the local storage units is used to serve the arriving demands. If at any time, the total demand exceeds this total available power, the arriving customers will be blocked. Blocking these customers will effectively isolate the peak demands from the electrical grid. The rate of the customers being blocked at the station could be used as a metric to evaluate the level of QoS of the charging station.

Eventually, to take into account different states of the electrical grid, we assume the price the price of each unit of electrical power (reflecting the state of the grid at that moment) is fluctuating during the day according to some rates. These prices are sent to the station in real time without delay. In computing the cost of utilizing the station, we will consider these price fluctuations.

\subsection{Charging Station Model: Mathematical details}

In the previous section, we introduced a model for a charging station of EVs. Here, we explain this model with mathematical details.

\subsubsection{System primitives}

We assume vehicles arriving to a charging station, each carrying a certain charging demand. The arrivals are assumed to be Poisson with rate $\lambda$. Even for EVs equipped with the same type of battery, they could come with different battery size and/or different energy demands. Therefore, we assume that the demands are independently and identically distributed (i.i.d) exponential random variables with rate $\mu$ (As mentioned in Section 5.1, the electrical power is quantized by the number of cars being 
charged.). ${ }^{1}$

The charging station can draw the electrical power either directly from the grid or from a local storage facility (a battery). At any time, the grid is able to charge at most $S$ cars. By level of the battery, we mean the number of cars that can be simultaneously charged from the battery at a given time. The level fluctuates between 0 , indicating that the battery is empty, and $R$ (the capacity of the battery) - the battery is fully charged. Both $S$ and $R$ are fixed/given deterministic parameters of the model. Let $i=i(t)$ denote the number of cars in the charging station at time $t$ and $j=j(t)$ be the level of the battery at time $t$. The station cannot have more than $S+j$ cars being charged at any given time, which is regulated by blocking the newly arrived cars. This implies

$$
0 \leq j \leq R \quad \text { and } \quad 0 \leq i \leq S+j
$$

At any given time, a car can be served either by the grid or the battery. Letting $i_{S}$ and $i_{R}$ represent the number of cars being charged by the grid and battery respectively, we have

$$
0 \leq i_{S} \leq S \quad \text { and } \quad 0 \leq i_{R} \leq j \quad \text { and } \quad i_{R}+i_{S}=i
$$

If $i_{S}<S$, at some point of time, the remaining $S-i_{S}$ unused power units of the grid could be used to charge the battery, if it is not fully charged, i.e. $j<R$. The charging time of each level of battery when using a unit of electrical power is a random variable exponentially distributed with rate $\mu$, which is independent of charging times of other levels of battery and also independent of charging times of EVs. Therefore, we assume that the charging times of each EV and each level of battery are i.i.d exponential random variables with the same rate $\mu$. If the battery is being charged using $\left(S-i_{S}\right)$ units from the power grid, the charging rate of each level of battery is equal to: $\min \left(\left(S-i_{S}\right), S_{P R}\right) \cdot \mu$, where $S_{P R}$ is the power rating of the battery. In the sequel, to simplify our notations, without loss of generality, we assume that $S \leq S_{P R}$. So, in case of charging the battery using $\left(S-i_{S}\right)$ units of power, the charging rate of each level of battery is: $\left(S-i_{S}\right) \mu$.

\footnotetext{
${ }^{1}$ The Markovian/memoryless assumptions for the arrival and demands distributions, although may not be entirely realistic, they will nevertheless enable us to model our problem as a Markov Decision Process (MDP); this would not be possible for general arrival and demand distributions. We believe this approach may provide useful intuition to similar practical problems.
} 


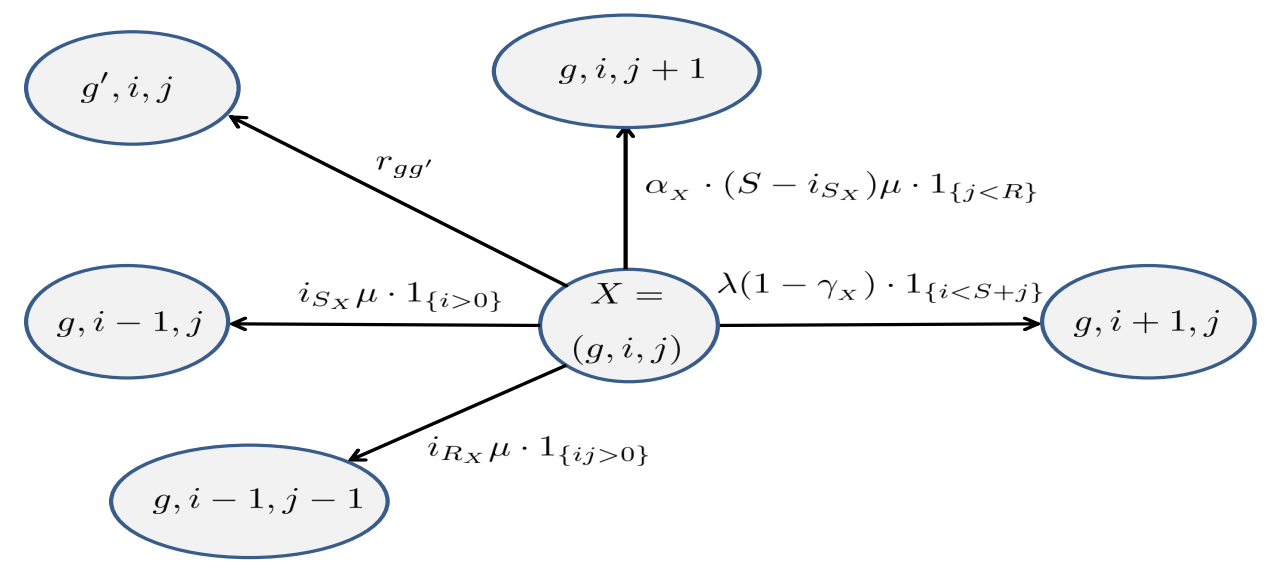

Figure 5.2: The out-flow of the state $g, i, j$ in the charging station

The state of the charging station at each time $t$, is determined by a threedimensional vector $X=(g, i, j)$, with $i$ and $j$ are defined as above. An additional variable $g$ denotes the price of each unit of electrical power in the grid, supporting our assumption about RTP scheme for pricing. In particular, we assume that that $g \in G$, where $G$, the power unit price set, is a finite discrete set composed of all possible values of $g$. Furthermore, assume that the time the grid remains in each of the states from $G$ is an exponential random variable with rate $r_{g}=\sum_{g^{\prime} \neq g} r_{g g^{\prime}}$, where $r_{g g^{\prime}}$ is the rate of going from a state of price $g$ to one of price $g^{\prime}$. We assume that new updated prices are sent to the station using a high-speed connection without delay. Therefore, at each time $t$, the state of the system, $X$, belongs to the state space:

$$
\mathcal{X}=\{(g, i, j): g \in G, 0 \leq j \leq R, 0 \leq i \leq S+j\}
$$

The size of $\mathcal{X}$ is equal to the output of the following equation:

$$
|\mathcal{X}|=\sum_{g \in G} \sum_{j=0}^{R} \sum_{i=0}^{S+j} 1
$$




\subsubsection{Introducing controls}

The purpose of this section is to introduce controls, i.e., represent our model as a controlled Markov Process. At each time $t$, and state of the system $X=(g, i, j)$, we introduce a control vector $\mathbf{u}_{X, t}=\left(\gamma_{X, t}, i_{R_{X, t}}, \alpha_{X, t}\right)$ in the following manner. The controls will be applied at transition instances so that an appropriate cost (to be introduced) is optimized.

The control variable $\gamma_{x, t} \in[0,1]$ represents the probability of blocking arriving cars from entering the charging station. In particular, upon arrival of a new car the controller accepts this car with probability $\left(1-\gamma_{x, t}\right)$. Clearly, when $i=S+j$, the system does not have enough power to accept any new arrivals and the controller will block all new arriving cars. So, in these cases, we set $\gamma_{x, t} \equiv 1$.

The second control variable $\alpha_{X, t} \in[0,1]$ represents the proportion of available grid power to be used for charging the battery. In particular, when there are $\left(S-i_{S}\right)$ power grid units available, the battery charge rate will be equal to $\alpha_{x, t}\left(S-i_{S}\right) \mu$. Clearly, when $j=R$, the battery is fully charged already, in which case we assume $\alpha_{X, t} \equiv 0$.

The last control variable, $i_{R_{X, t}}$ serves to split the cars being charged in the station between the grid and the battery. When the number of cars being charged in the system is $i$, the action of the controller is to determine $i_{R}$ and $i_{S}$, with respect to the conditions in Equation (5.2). Note that when entering a new state $X$, the controller decides on the values of $i_{R}$ and $i_{S}$ independent of their values in previous states. In other words, there is no restriction to assign a car previously being charged by the grid, to be charged by the battery and vice versa. The value of of $i_{R}$ is bounded according to the following criteria: We have $\min \left(i_{R}\right)=(i-S)^{+}=\max (0, i-S)$, which is equivalent to sending all the cars being charged to the grid, with the remaining cars to be charged by the battery. On the other hand $\max \left(i_{R}\right)=\min (i, j)$, which is equivalent to sending cars being charged to the battery up to its charging level. The remaining cars will be charged by the grid. Therefore: $i_{R} \in\left\{(i-S)^{+}, \ldots, \min (i, j)\right\}$. Note that when $i=0$ or $j=0$, we have $i_{R_{X, t}} \equiv 0$ and $i_{S_{X, t}} \equiv i$.

Hence, for the control vector $\mathbf{u}_{X, t}$, we have: $\mathbf{u}_{X, t}=\left(\gamma_{X, t}, i_{R_{X, t}}, \alpha_{X, t}\right) \in \Omega_{X, t}$, where:

$$
\Omega_{X, t}=[0,1] \times\left\{(i-S)^{+}, \ldots, \min (i, j)\right\} \times[0,1]
$$

In the sequel, for simplicity in our notation, we denote $\Omega_{X, t}$ by $\Omega$. Figure 5.2 illustrates 
the transition diagram in the resulted Markov process. These rates form the generator matrix $Q$. The resulting controlled Markov process has a finite state-space $(\mathcal{X})$.

\subsection{Optimal Control Policy}

We now need to introduce an instantaneous cost function for our model. Given the state at time $t$ is $X_{t}=(g, i, j)$ and the selected action vector is $\mathbf{u}_{X, t}=\left(\gamma_{X, t}, i_{R_{X, t}}, \alpha_{X, t}\right)$. We define the operational grid cost incurred by the charging station during the time interval $[t, t+d t]$, as follows:

$$
C_{t}^{1}\left(X, \mathbf{u}_{X, t}\right) d t=\left(g i_{S_{X, t}}+g \alpha_{X, t}\left(S-i_{S_{X, t}}\right)-V_{X} i\right) d t
$$

where $V_{X}$ is the revenue obtained by the charging station per unit time from each EV being charged at the station. As we see in the equation above, its value depends on the state. For example, it could vary as the price of each unit of electrical power $(g)$ varies.

In addition, we are interested in taking into account the quality of service offered by the station, in terms of the number of blocked EVs. Let $C_{b l}$ be the cost incurred per each blocked EV. Then the corresponding blocking cost in the time interval $[t, t+d t]$ is:

$$
C_{t}^{2}\left(X, \mathbf{u}_{X, t}\right) d t=C_{b l_{X}} \lambda_{X} \gamma_{X, t} d t
$$

Both $C_{b l}$ and $\lambda$ are state dependent. For example, the rate of arrivals, $\lambda$, could change when the price of each unit of electrical power $(g)$ changes (So, it would reflect different rates of arrivals during rush/peak hours versus the rest of the day). Similarly, $C_{b l}$ could be different for different value of $g$. Also, it could be different for cases where blocking happens due to the lack of resources, i.e. when $i=S+j$, or it happens because of a controller's decision. In the sequel, to simplify our notations, without loss of generality, we drop the index $X$ for $C_{b l}$ and $\lambda$. 
Combining these two costs results in the following equation:

$$
\begin{aligned}
C_{t}\left(X, \mathbf{u}_{X, t}\right) d t & =\left(C_{t}^{1}\left(X, \mathbf{u}_{X, t}\right)+C_{t}^{2}\left(X, \mathbf{u}_{X, t}\right)\right) d t \\
& =\left(g \cdot i_{S_{X, t}}+g \cdot \alpha_{X, t} \cdot\left(S-i_{S_{X, t}}\right)\right. \\
& \left.-V_{X} \cdot i+C_{b l} \lambda \gamma_{X, t}\right) d t
\end{aligned}
$$

This cost function is stationary and independent of the time. So at each time $t$, we can write:

$$
C_{t}\left(X, \mathbf{u}_{X, t}\right) d t=C\left(X, \mathbf{u}_{X, t}\right) d t
$$

A control policy is a set of decision rules $\mathbf{z}=\left\{\mathbf{u}_{X, t}=\left(\gamma_{X, t}, i_{R_{X, t}}, \alpha_{X, t}\right) \in \Omega\right.$ : $X \in \mathcal{X}, t\}$. Denote by $\mathcal{Z}$ the set of all policies. We are interested in finding a policy minimizing the average incurred cost during the operating time of the system. In the sequel, we assume that the system operates over an infinite horizon time $[0, \infty)$.

Based on the previous definitions, our problem can be modelled as a Markov Decision Process (MDP). Following standard practice in Markov Decision Processes, we will introduce a finite horizon $\delta$-discounted cost problem and find its optimal control policy. Then the optimal policy of the infinite horizon problem can be derived as a limit of the optimal policies of a sequence of $\delta$-discounted problems [75]. Let $\delta$ be the discount rate. Then given the initial state is $X$ and the time horizon is $\{t: t>0\}$, $\mathbf{J}_{t}^{\delta}(X)$, the minimum expected total $\delta$-discounted cost is defined as follows:

$$
\mathbf{J}_{t}^{\delta}(X)=\min _{\mathbf{z} \in \mathcal{Z}} \mathbf{E}_{\mathbf{X}}^{\mathbf{z}}\left(\int_{0}^{t} e^{-\delta \tau} C\left(X_{\tau}, \mathbf{u}_{X, \tau}\right) d \tau\right)
$$

So the infinite horizon optimal $\delta$-discounted expected cost is:

$$
\mathbf{J}^{\delta}(X)=\min _{\mathbf{z} \in \mathcal{Z}} \mathbf{E}_{\mathbf{X}}^{\mathbf{z}}\left(\int_{0}^{\infty} e^{-\delta \tau} C\left(X_{\tau}, \mathbf{u}_{X, \tau}\right) d \tau\right)
$$

If there exists a policy $\mathbf{z}^{*}$, resulting in $\mathbf{J}^{\delta}(X)$, this policy is called the optimal $\delta$ discounted expected cost policy. This optimal policy satisfies the following optimality 
condition [76]:

$$
\begin{aligned}
& \mathbf{J}_{t+d t}^{\delta}(X)=\min _{\mathbf{u}_{X, 0} \in \Omega}\left\{C\left(X, \mathbf{u}_{X, 0}\right) d t\right. \\
+ & \left.e^{-\delta d t} d t\left(\sum_{X^{\prime} \in \mathcal{X}} \mathbf{P}\left(X_{d t}=X^{\prime} \mid X_{0}=X, \mathbf{u}_{X, 0}\right) \mathbf{J}_{t}^{\delta}\left(X^{\prime}\right)\right)\right\}
\end{aligned}
$$

Lemma 8 For the cost function defined in (5.7), the optimal policy $\mathbf{z}^{*}$ with respect to equation (5.10) exists and it is stationary.

Proof.The state space $\mathcal{X}$ is finite and the resulting Markov chain (Figure 5.2) is irreducible. In addition, since we assumed that $S$ and $R$ are finite values, (Section $5.2)$, the transition rates are finite and all of these transition rates are independent of time. Moreover from Equation (5.8), we know that the cost function $C\left(X, \mathbf{u}_{X, t}\right)$ is independent of time. Therefore, the optimal policy exists and it is stationary [75]; that is at time $t$, when the state of the system is $X_{t}=X$, the optimal action exists, is independent of $t$ and only depends on the state of the system, $X$. So we have:

$$
\mathbf{z}^{*}=\left\{\mathbf{u}_{X, t}^{*}=\mathbf{u}_{X}^{*}: \quad X \in \mathcal{X}, \quad t\right\}
$$

\subsubsection{Deriving the Optimal Policy}

So far, it has been shown the optimal policy exists and it is stationary. In this subsection, we show how to find the optimal policy. To do so, we first relate the continuous time Markov chain $\left\{X_{t}: t \geq 0\right\}$ to a suitable discrete time Markov chain. Then, we find the corresponding cost function and transition probabilities for the resulting discrete time Markov chain. Eventually, we will apply them to the corresponding discrete time dynamic programming equations to find the optimal action for each state and hence the optimal policy.

By using the method of "uniformization" [74], we can relate the continuous time Markov chain $\left\{X_{t}: t \geq 0\right\}$ to a suitable discrete time chain $\left\{X_{k}: k \geq 0\right\}$. To do so, first we define the "total event rate" as follows:

$$
\rho=\lambda+(S+R) \mu+\sum_{g \in G} r_{g}
$$


Let $0=t_{0}<t_{1}<t_{2}<\cdots<t_{n}<\cdots$ be the transition epochs with respect to possible transitions defined in the original Markov Chain shown in Figure 5.2. By suitably introducing "dummy" transitions, it follows that the inter-transition intervals are i.i.d exponential random variables with rate $\rho$. Then, it can be shown [74] that for a policy $\mathbf{z} \in \mathcal{Z}$, the $\delta$-discounted expected cost up to time $t_{n}$, i.e.: $\mathbf{E}_{X}^{\mathbf{z}}\left(\int_{0}^{t_{n}} e^{-\delta \tau} C\left(X_{\tau}, \mathbf{u}_{X, \tau}\right) d \tau\right)$ is equal to a cost:

$$
\mathbf{E}_{X}^{\mathbf{z}}\left(\sum_{k=0}^{n-1} \beta^{k} C_{d}\left(X_{k}, \mathbf{u}_{X, k}\right)\right)
$$

where $\left\{X_{k}: k \geq 0\right\}$ is the discrete time Markov chain obtained from $\left\{X_{t}: t \geq 0\right\}$ by using the uniformization technique, such that $X_{k} \triangleq X_{t_{k}}$. Equivalently $\mathbf{u}_{X, k} \triangleq \mathbf{u}_{X, t_{k}}$ is the action at $k$-th transition, where the discrete time Markov chain is at the state $X$. Furthermore, in equation (5.14), $\beta \triangleq \frac{\rho}{\rho+\delta}<1$. Finally, $C_{d}\left(X_{k}, \mathbf{u}_{X, k}\right)$ is the discrete version of $C\left(X_{\tau}, \mathbf{u}_{X, \tau}\right)$ and defined as follows:

$$
\begin{aligned}
C_{d}\left(X_{k}, \mathbf{u}_{X, k}\right) & =g i_{S_{X, k}}+g \alpha_{X, k}\left(S-i_{S_{X, k}}\right) \\
& -V_{X} i_{X}+\lambda \gamma_{X, k} C_{b l}
\end{aligned}
$$

The expectation in (5.14) is taken with respect to the probability distribution associated to the discrete time Markov chain $\left\{X_{k}: \quad k \geq 0\right\}$, when the control policy $\mathbf{z}$ is used. The resulted transition probabilities are as follows:

$$
\begin{gathered}
\mathbb{P}^{\mathbf{z}}\left(X_{k+1}=\left(g^{\prime}, i^{\prime}, j^{\prime}\right) \mid X_{k}=(g, i, j), \mathbf{u}_{x, k}=\left(\gamma, i_{R}, \alpha\right)\right) \cdot \rho \\
= \begin{cases}\lambda(1-\gamma) \cdot 1_{\{i<S+j\}} & g^{\prime}=g, i^{\prime}=i+1, j^{\prime}=j \\
r_{g g^{\prime}} & g^{\prime} \neq g, i^{\prime}=i \quad, j^{\prime}=j \\
i_{R} \mu 1_{\{i j>0\}} & g^{\prime}=g, i^{\prime}=i-1, j^{\prime}=j-1 \\
i_{S} \mu 1_{\{i>0\}} & g^{\prime}=g, i^{\prime}=i-1, j^{\prime}=j \\
\alpha\left(S-i_{S}\right) \mu \cdot 1_{\{j<R\}} & g^{\prime}=g, i^{\prime}=i+1, j^{\prime}=j \\
\rho-\rho^{\prime} & g^{\prime}=g, i^{\prime}=i \quad, j^{\prime}=j\end{cases}
\end{gathered}
$$


Where

$$
\begin{aligned}
\rho^{\prime} & =\lambda(1-\gamma) \cdot 1_{\{i<S+j\}}+\sum_{g^{\prime} \neq g} r_{g g^{\prime}}+i_{R} \mu 1_{\{i j>0\}} \\
& +i_{S} \mu 1_{\{i>0\}}+\alpha\left(S-i_{S}\right) \mu \cdot 1_{\{j<R\}}
\end{aligned}
$$

For each initial state $X \in \mathcal{X}$, we can define the $n$-step optimal $\beta$-discounted expected cost as follows: $\mathbf{J}_{n}^{\beta}(X)=\min _{\mathbf{z} \in \mathcal{Z}} \mathbb{E}_{X}^{\mathbf{z}}\left(\sum_{k=0}^{n-1} \beta^{k} C_{d}\left(X_{k}, \mathbf{u}_{X, k}\right)\right)$. Then, the infinite horizon optimal $\beta$-discounted expected cost is defined as follows:

$$
\mathbf{J}^{\beta}(X)=\min _{\mathbf{z} \in \mathcal{Z}} \mathbb{E}_{X}^{\mathbf{z}}\left(\sum_{k=0}^{\infty} \beta^{k} C_{d}\left(X_{k}, \mathbf{u}_{X, k}\right)\right)
$$

Since the state space $\mathcal{X}$ is finite, it can be shown [75] that $\lim _{n \rightarrow \infty} \mathbf{J}_{n}^{\beta}(X)=\mathbf{J}^{\beta}(X)$. Furthermore, the optimal policy exists and it is stationary.

Theorem 5 In the optimal policy $\mathbf{z}^{*}$, the set of all admissible actions for $\mathbf{u}_{X}^{*}$ has the form $\mathcal{A}=\{0,1\} \times\left\{(i-S)^{+}, \min (i, j)\right\} \times\{0,1\}$. In other words, the optimal policy actions attain the extreme values in their corresponding sets. Such policies are known as bang-bang policies.

To prove Theorem 5, we first derive the dynamic programming equations for discrete Markov chain $X$ and perform the necessary minimization with respect to $\gamma$, $i_{R}$ and $\alpha$.

Proof.The optimal policy is obtained from the discrete time formulation of the dynamic programming equation [74], as follows:

$$
\begin{aligned}
\mathbf{J}_{k+1}^{\beta}(X) & =\min _{\mathbf{u}_{X, 0} \in \Omega}\left\{C_{d}\left(X, \mathbf{u}_{X, 0}\right)\right. \\
& \left.+\beta\left(\sum_{X^{\prime} \in \mathcal{X}} \mathbf{P}\left(X_{1}=X^{\prime} \mid X_{0}=X, \mathbf{u}_{X, 0}\right) \mathbf{J}_{k}^{\beta}\left(X^{\prime}\right)\right)\right\}
\end{aligned}
$$

Where $\mathbf{u}_{X, 0}$ denotes the chosen action at $k=0$, while the the system is in initial state $X$. However, since the optimal policy is stationary, for each $X \in \mathcal{X}$ we have: $\mathbf{u}_{X, 0}=\mathbf{u}_{X}$. Now, we can expand Equation (5.19), using $\mathbf{u}_{X}=\left(\gamma_{X}, i_{R_{X}}, \alpha_{X}\right)$. For simplicity in our notations, in the following equations, we replace $\left(\gamma_{X}, i_{R_{X}}, \alpha_{X}\right)$ with 
$\left(\gamma, i_{R}, \alpha\right)$. Performing some easy algebra results in:

$$
\begin{aligned}
& \mathbf{J}_{k+1}^{\beta}(X)= \\
& \min _{\gamma \in[0,1]}\left\{\lambda \gamma\left(C_{b l}-\frac{\beta}{\rho} 1_{\{i<S+j\}}\left(\mathbf{J}_{k}^{\beta}(g, i+1, j)-\mathbf{J}_{k}^{\beta}(g, i, j)\right)\right)\right\} \\
+ & \min _{i_{R} \in\left\{(i-S)^{+}, \cdots, \min (i, j)\right\}, \alpha \in[0,1]}\{ \\
& i_{S}\left(g+\frac{\beta \mu}{\rho} 1_{\{i>0\}}\left(\mathbf{J}_{k}^{\beta}(g, i-1, j)-\mathbf{J}_{k}^{\beta}(g, i, j)\right)\right. \\
+ & i_{R} \frac{\beta \mu}{\rho} 1_{\{i j>0\}}\left(\mathbf{J}_{k}^{\beta}(g, i-1, j-1)-\mathbf{J}_{k}^{\beta}(g, i, j)\right) \\
+ & \left.\alpha\left(S-i_{S}\right)\left(g+\frac{\beta \mu}{\rho} 1_{\{j<R\}}\left(\mathbf{J}_{k}^{\beta}(g, i, j+1)-\mathbf{J}_{k}^{\beta}(g, i, j)\right)\right)\right\} \\
+ & \text { terms not depending on }\left(\gamma, i_{R}, \alpha\right)
\end{aligned}
$$

Equation (5.20) shows that the optimal choice for $\gamma$ doesn't depend on $\alpha$ and $i_{R}$. Also, we know that if $i=S+j$, then $\gamma \equiv 1$. So, when $i<S+j$, the optimal choice for $\gamma$ is obtained as follows:

$$
\gamma= \begin{cases}0 & \left(C_{b l} \rho-\beta\left(\mathbf{J}_{k}^{\beta}(g, i+1, j)-\mathbf{J}_{k}^{\beta}(g, i, j)\right)\right) \geq 0 \\ 1 & \left(C_{b l} \rho-\beta\left(\mathbf{J}_{k}^{\beta}(g, i+1, j)-\mathbf{J}_{k}^{\beta}(g, i, j)\right)\right)<0\end{cases}
$$

To find the optimal choices for $\alpha$ and $i_{R}$, we should consider the second part of the Equation (5.20). First assume that $i \cdot j=0$. Then as it is discussed in Section 5.2, $i_{R}=0$ and $i_{S}=i$. Also, we know that if $j=R$, then $\alpha \equiv 0$. From Equation (5.2), we have: $\left(S-i_{S}\right) \geq 0$, independent of our choice for $i_{R}$. Therefore, when $i \cdot j=0$ and $j<R$, by using Equation (5.20), the optimal choice for $\alpha$ is as follows:

$$
\alpha= \begin{cases}0 & \left(g \rho+\beta \mu\left(\mathbf{J}_{k}^{\beta}(g, i, j+1)-\mathbf{J}_{k}^{\beta}(g, i, j) \quad\right) \geq 0\right. \\ 1 & \left(g \rho+\beta \mu\left(\mathbf{J}_{k}^{\beta}(g, i, j+1)-\mathbf{J}_{k}^{\beta}(g, i, j)\right)\right)<0\end{cases}
$$

Now, consider a case when $i>0$ and $j=R$. Hence $\alpha \equiv 0$. Consequently, by using 
Equation (5.20), the optimal choice for $i_{R}$ is as follows:

$$
i_{R}=\left\{\begin{array}{rr}
(i-S)^{+} & -g \rho+\beta \mu \mathbf{J}_{k}^{\beta}(g, i-1, j-1) \\
& -\beta \mu \mathbf{J}_{k}^{\beta}(g, i-1, j) \geq 0 \\
\min (i, j) & -g \rho+\beta \mu \mathbf{J}_{k}^{\beta}(g, i-1, j-1) \\
& -\beta \mu \mathbf{J}_{k}^{\beta}(g, i-1, j)<0
\end{array}\right.
$$

The most general case happens when $i \cdot j>0$ and $j<R$. In this case, Equation (5.20) becomes as follows:

$$
\begin{aligned}
& \mathbf{J}_{k+1}^{\beta}(X)=\min _{i_{R}, \alpha}\{ \\
& i_{R}\left(-g+\frac{\beta \mu}{\rho}\left(\mathbf{J}_{k}^{\beta}(g, i-1, j-1)-\mathbf{J}_{k}^{\beta}(g, i-1, j)\right)\right. \\
+ & \left.\alpha\left(S-i_{S}\right)\left(g+\frac{\beta \mu}{\rho}\left(\mathbf{J}_{k}^{\beta}(g, i, j+1)-\mathbf{J}_{k}^{\beta}(g, i, j)\right)\right)\right\} \\
+ & \text { terms not depending on }\left(i_{R}, \alpha\right)
\end{aligned}
$$

As we can see in Equation (5.24), only the second term depends on $\alpha$. Now since $\left(S-i_{S}\right) \geq 0$ (Equation (5.2)), in order to minimize the RHS of Equation (5.24) with respect to $\alpha$, we should minimize the following expression: $\alpha\left(S-i_{S}\right)\left(g+\frac{\beta \mu}{\rho}\left(\mathbf{J}_{k}^{\beta}(g, i, j+\right.\right.$ 1) $\left.-\mathbf{J}_{k}^{\beta}(g, i, j)\right)$ ), which results in using the same criteria as Equation (5.22). Now, if the criteria in Equation (5.22) result in $\alpha=0$, Equation (5.24) becomes as follows:

$$
\begin{aligned}
& \quad \mathbf{J}_{k+1}^{\beta}(X)=\min _{i_{R}}\{ \\
& \quad i_{R}\left(-g+\frac{\beta \mu}{\rho}\left(\mathbf{J}_{k}^{\beta}(g, i-1, j-1)-\mathbf{J}_{k}^{\beta}(g, i-1, j)\right)\right. \\
& + \text { terms not depending on }\left(i_{R}\right)
\end{aligned}
$$

Equation (5.25) tells us that using the same criteria as Equation (5.23) gives us the optimal value of $i_{R}$, which minimizes Equation (5.24). On the other hand, if using the 
criteria in Equation (5.22) results in $\alpha=1$, then Equation (5.24) becomes as follows:

$$
\begin{aligned}
& \mathbf{J}_{k+1}^{\beta}(X)= \\
& \quad \min _{i_{R}}\left\{i _ { R } \frac { \beta \mu } { \rho } \left(\mathbf{J}_{k}^{\beta}(g, i-1, j-1)-\mathbf{J}_{k}^{\beta}(g, i-1, j)\right.\right. \\
& \left.\left.\left.+\quad \mathbf{J}_{k}^{\beta}(g, i, j+1)-\mathbf{J}_{k}^{\beta}(g, i, j)\right)\right)\right\} \\
& + \text { terms not depending on }\left(i_{R}, \alpha\right)
\end{aligned}
$$

Therefore, when $i \cdot j>0$ and $j<R$, if using criteria in Equation (5.22) results in $\alpha=1$, to find the optimal choice for $i_{R}$, we should use the following criteria:

$$
i_{R}= \begin{cases}(i-S)^{+} & \left(\mathbf{J}_{k}^{\beta}(g, i-1, j-1)-\mathbf{J}_{k}^{\beta}(g, i-1, j)\right. \\ & \left.\left.+\mathbf{J}_{k}^{\beta}(g, i, j+1)-\mathbf{J}_{k}^{\beta}(g, i, j)\right)\right) \geq 0 \\ \min (i, j) & \left(\mathbf{J}_{k}^{\beta}(g, i-1, j-1)-\mathbf{J}_{k}^{\beta}(g, i-1, j)\right. \\ & \left.\left.+\mathbf{J}_{k}^{\beta}(g, i, j+1)-\mathbf{J}_{k}^{\beta}(g, i, j)\right)\right)<0\end{cases}
$$

Equations (5.21), (5.22), (5.23) and (5.27) show that for every state $X \in \mathcal{X}$, there exists a set of admissible actions $\mathcal{A}_{X}$, as follows:

$$
\mathcal{A}_{X}=\{0,1\} \times\left\{(i-S)^{+}, \min (i, j)\right\} \times\{0,1\}
$$

such that $\mathbf{u}_{X}=\left(\gamma_{X}, i_{R_{X}}, \alpha_{X}\right) \in \mathcal{A}_{X}$ (Similarly to what we did for $\Omega$, for simplicity in our notation, in the sequel, we denote $\mathcal{A}_{X}$ by $\mathcal{A}$ ). Denote by $\mathcal{Z}^{\mathcal{A}}$, the set of all admissible policies. Then, for each $\mathbf{z}^{\mathcal{A}} \in \mathcal{Z}^{\mathcal{A}}$, including $\mathbf{z}^{*}$, we have: $\mathbf{z}^{\mathcal{A}}=\left\{\mathbf{u}_{x} \in \mathcal{A}\right.$ : $X \in \mathcal{X}\}$. Therefore, instead of initial action set $\Omega$ (Equation (5.5)), we are sure that the actions in the optimal policy belong to a much smaller set $\mathcal{A}$, which is indeed the boundaries of the initial set. In the literature, such policies are called "bang-bang" policies. So, $\mathbf{z}^{*}$ exists and is a bang-bang policy.

Using dynamic programming equation (5.20) iteratively along with the criteria in optimal policy in Section 5.3, we will find the infinite horizon optimal $\beta$-discounted expected $\operatorname{cost} \mathbf{J}^{\beta}(X)$ and its corresponding optimal policy $\mathbf{z}^{*} \in \mathbf{Z}^{\mathbf{A}}$, with finite number of iterations [74]. 


\subsubsection{Expected total $\beta$-discounted cost}

$C_{d}\left(X, \mathbf{u}_{X}\right)$ (Equation (5.15)) is the cost of the discrete-time Markov chain $\left\{X_{k}: k \geq\right.$ $0\}$, when it is in the state $X \in \mathcal{X}$ and action $\mathbf{u}_{X, k}$ is determined by stationary policy z. The $\beta$-discounted expected cost of a stationary and time-invariant policy $\mathbf{z}$, when the initial state is $X \in \mathcal{X}$, is computed as follows:

$$
\mathbf{J}_{\mathbf{z}}^{\beta}(X)=\mathbb{E}_{X}^{\mathbf{Z}}\left(\sum_{k=0}^{\infty} \beta^{k} C_{d}\left(X_{k}, \mathbf{u}_{X, k}\right)\right)
$$

Define the column vectors $\mathbb{J}_{\mathbf{z}}^{\beta}$ and $\mathbb{C}_{\mathbf{z}}$ of size $|\mathcal{X}|$, where their elements are $\mathbf{J}_{\mathbf{z}}^{\beta}(X)$ and $C_{d}\left(X, \mathbf{u}_{X}\right)$ respectively. In [74](Page 43, Chapter 4), it is shown that for a stationary policy $\mathbf{z}$, the set of $|\mathcal{X}|$ linear equations:

$$
\left(\mathbb{I}_{|\mathcal{X}|}-\beta \mathbb{P}^{\mathbf{z}}\right) \mathbb{J}_{\mathbf{z}}^{\beta}=\mathbb{C}_{\mathbf{z}}
$$

has a unique solution. It assures us that for a stationary policy $\mathbf{z}$ and for each state $X \in \mathcal{X}$, the Equation (5.29) will definitely converge to a unique value. So for each stationary and time-invariant policy $\mathbf{z}$, including the optimal policy $\mathbf{z}^{*}$, we can compute the expected total $\beta$-discounted cost as follows:

$$
\mathbf{J}_{\mathbf{z}}^{\beta}(\mathbb{X})=\mathbb{E}_{\mathbb{X}}^{\mathbf{z}} \mathbf{J}_{\mathbf{z}}^{\beta}(X)
$$

The Expectation in Equation (5.31) is computed with respect to a given initial distribution $\mathbb{X}$. Therefore, to compute the expected total $\beta$-discounted cost of a stationary and time-invariant policy $\mathbf{z}$, instead of using Equation (5.29), we can use linear Equation (5.30) along with Equation (5.31). In Subsection 5.3.4, we compare the expected total $\beta$-discounted cost of the optimal policy $\mathbf{z}^{*}$ with that of certain other policies.

\subsubsection{Minimizing the infinite horizon Average Cost}

For each stationary policy $\mathbf{z}=\left\{\mathbf{u}_{X} \in \Omega: X \in \mathcal{X}\right\}$, where we have $\mathbf{u}_{X, t}=\mathbf{u}_{X}$ for each $X \in \mathcal{X}$, its equivalent stationary discrete time Markov chain (Section 5.3) is irreducible. Moreover, since the state space $\mathcal{X}$ is finite (Section 5.2), it is positive recurrent and hence ergodic [73]. Therefore, the steady state probability distribution $\pi=\{\pi(X): \quad X \in \mathcal{X}\}$ exists [73]- [74], and is the solution of $\pi=\pi \mathbf{P}$ subject to 
$\pi \cdot \mathbf{1}=1$, where $\mathbf{P}$ is the transition probability matrix for the resulting discrete time Markov chain $\left\{X_{k}^{\mathbf{z}}: k \geq 0\right\}$ and $\mathbf{1}$ is a column vector of size $|\mathcal{X}|$ (Equation (5.4)) with all elements equal to 1 . Similarly, for the optimal policy $\mathbf{z}^{*}=\left\{\mathbf{u}_{X}^{*} \in \mathcal{A}: X \in \mathcal{X}\right\}$ in Section 5.3 , by finding each action vector $\mathbf{u}_{X}^{*}$ for each state $X \in \mathcal{X}$, the transition probability matrix $\mathbb{P}^{z^{*}}$ in (5.16) is fully determined.

For each policy $\mathbf{z}$, the average cost per unit time incurred by the discrete Markov chain $\left\{X_{k}^{\mathbf{z}}: k \geq 0\right\}$ is calculated as follows:

$$
\mathbf{J}(\mathbf{z})=\lim _{N \rightarrow \infty} \frac{1}{N} \mathbb{E}^{\mathbf{z}} \sum_{k=0}^{N-1} C_{d}\left(X_{k}, \mathbf{u}_{X, k}\right)
$$

where $\mathbf{u}_{X, k}$ is determined by the policy $\mathbf{z}$. Since the Markov chain is ergodic, for the average cost per unit time in Equation (5.32), we get:

$$
\mathbf{J}(\mathbf{z})=\mathbb{E}^{\mathbf{z}} C_{d}\left(X, \mathbf{u}_{X}\right)
$$

In [74], it is shown that for the corresponding continuous time Markov Decision Processes, $\left\{X_{t}: t>0\right\}$, we have:

$$
\lim _{T \rightarrow \infty} \frac{1}{T} \mathbb{E}^{\mathbf{z}} \int_{0}^{T} C\left(X_{t}, \mathbf{u}_{X, t}\right) d t=\mathbf{J}(\mathbf{z})
$$

Furthermore, it is shown that as $\beta \rightarrow 1$, the optimal $\beta$-discounted expected cost policy $\mathbf{z}_{\beta}^{*}$, is then average cost optimal. Therefore the optimal average cost value is:

$$
\mathbf{J}^{*}=\lim _{\beta \rightarrow 1} \mathbb{E}^{\mathbf{z}_{\beta}^{*}} C_{d}\left(X, \mathbf{u}_{X}\right)
$$

where the expectation in (5.35) is taken with respect to the stationary probability distribution associated to the discrete time Markov chain $\left\{X_{k}^{*}: k \geq 0\right\}$ obtained from the optimal policy $\mathbf{z}_{\beta}^{*}$ with transition probabilities obtained from Equation (5.16).

\subsubsection{Numerical Examples}

In this Section, we first show the action-set in an optimal policy derived based on the recursive Equation (5.19) along with Equations (5.21), (5.22), (5.23) and (5.27) (All are rewritten in Table 5.1). Then, we will illustrate the final cost/revenue of 
Table 5.1: The summary of all Optimal Action Criteria

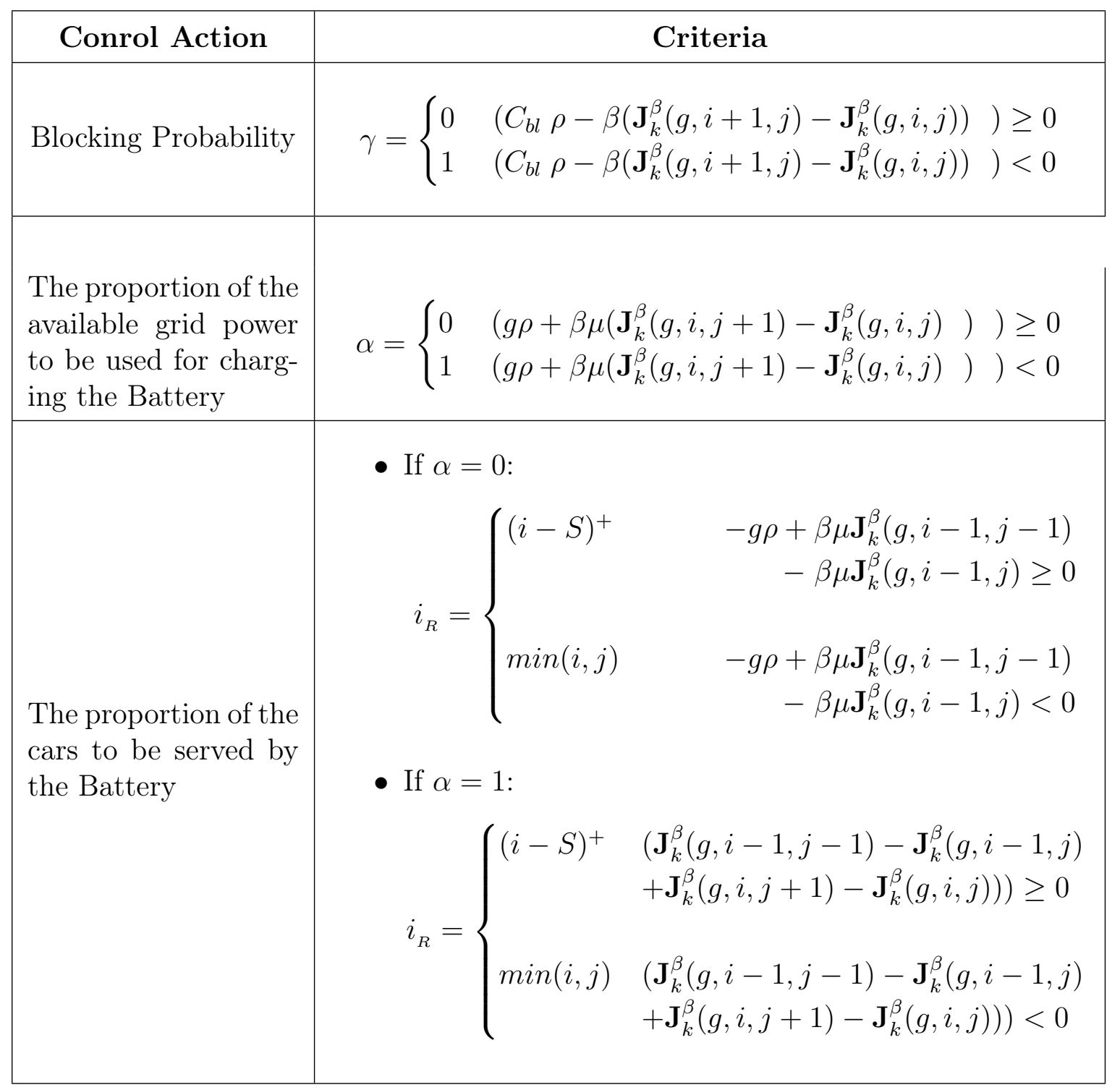

deploying such policy. The parameters of the charging station are given as follows: $S=6, R=6, \lambda=12, \mu=3, V=5.5$ and $C_{b l}=0.05$. In addition, the given power unit price set is: $G=\{3,5,10\}$. All of these unit prices are assumed to have the same mean times, e.g. 8 hours in a day, and equal transition probabilities to all other unit prices, i.e. $\frac{1}{2}$ in our setting. Therefore the transition rate between different unit 

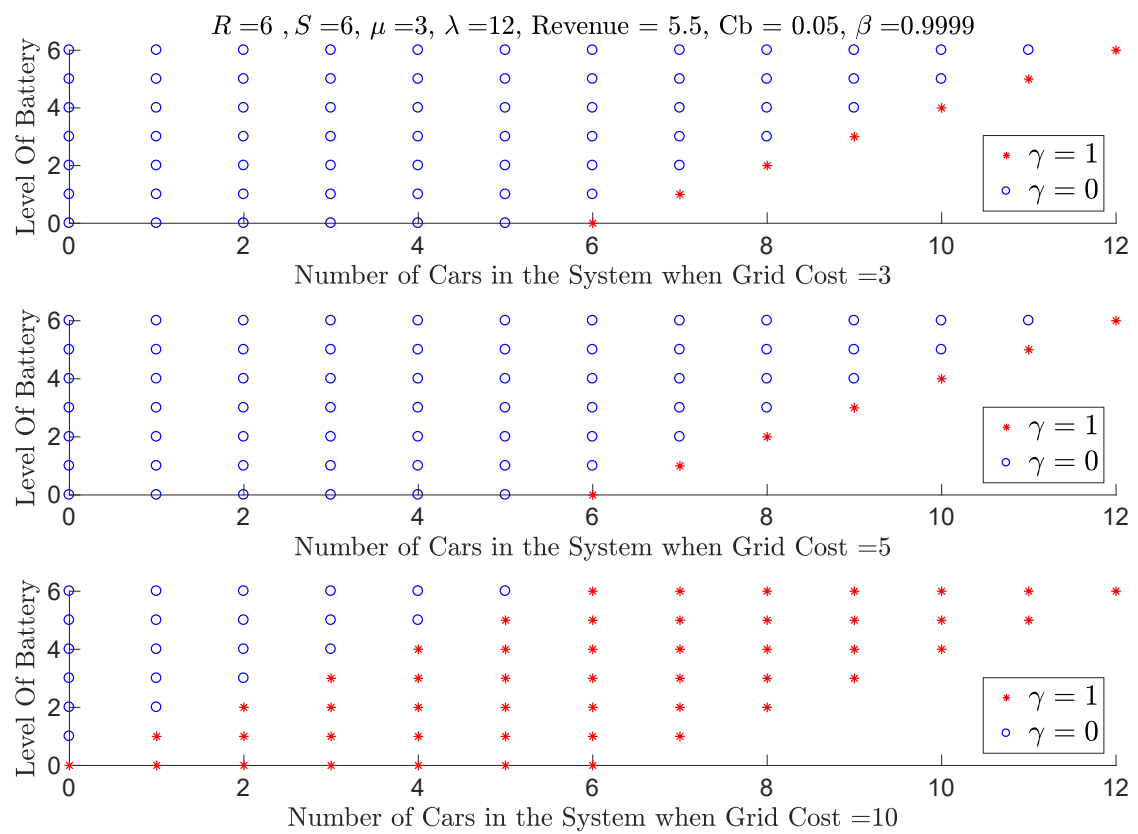

Figure 5.3: $\gamma$ in optimal policy $z^{*}$ obtained from dynamic programming equations, where $(o)$ and $(*)$ correspond to $\gamma=0$ and $\gamma=1$ respectively

prices is as follows: $r_{g g^{\prime}}=\frac{1}{16}$ for all $g \neq g^{\prime}$. Also it is assumed that $\beta=0.9999$.

Figures 5.3, 5.4 and 5.5 illustrate the values of $\gamma, \alpha_{X}$ and $i_{R}$ respectively in the optimal policy obtained from dynamic programming equations (5.20), using the criteria in optimal policy in Section 5.3. Generally, these figures give us some intuition about the optimal action for each state in the optimal policy. For example, we expect that for $X=(g, i, j)$ and $X^{\prime}=\left(g^{\prime}, i^{\prime}, j^{\prime}\right)$, if $\alpha_{X}=1\left(\right.$ or $\left.i_{R_{X}}=(i-S)^{+}\right)$, it is more likely that $\alpha_{X^{\prime}}=1\left(\right.$ or $\left.i_{R_{X^{\prime}}}=\left(i^{\prime}-S\right)^{+}\right)$, if:

- $i=i^{\prime}, j=j^{\prime}$ and $g^{\prime}<g$.

- $g=g^{\prime}, i=i^{\prime}$ and $j^{\prime}<j$.

- $g=g^{\prime}, j=j^{\prime}$ and $i^{\prime}>i$.

On the other hand, we expect that, if $\gamma_{X}=1$, it is more likely that $\gamma_{X^{\prime}}=1$, if:

- $i=i^{\prime}, j=j^{\prime}$ and $g^{\prime}>g$.

- $g=g^{\prime}, i=i^{\prime}$ and $j^{\prime}<j$. 

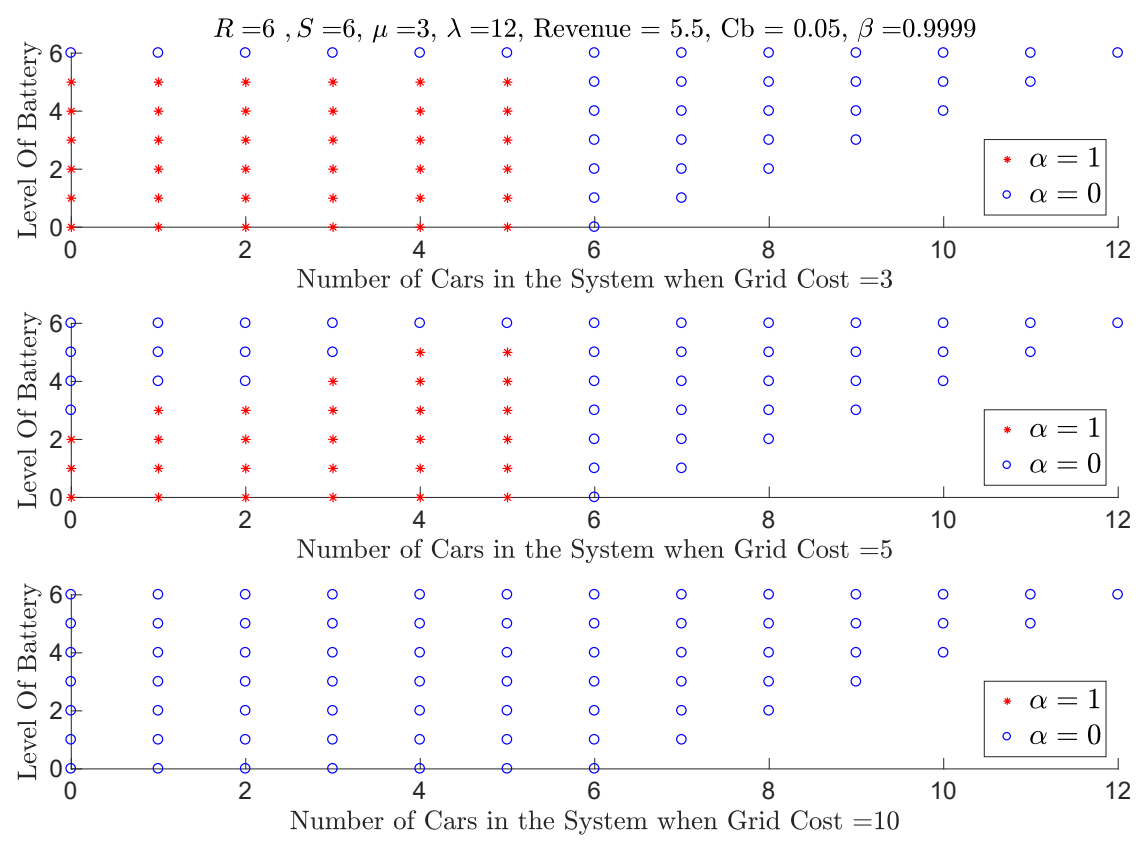

Figure 5.4: $\alpha$ in optimal policy $z^{*}$ obtained from dynamic programming equations, where $(o)$ and $(*)$ correspond to $\alpha=0$ and $\alpha=1$ respectively
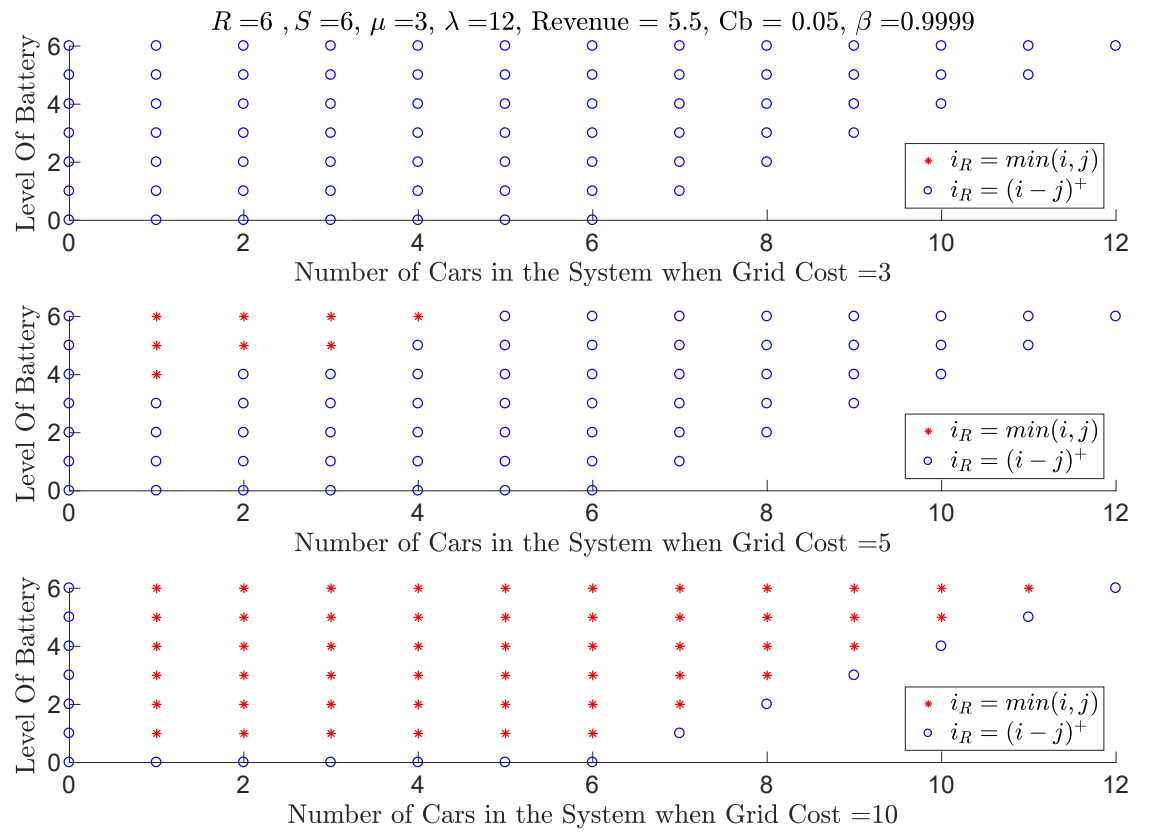

Figure 5.5: $i_{R}$ in optimal policy $z^{*}$ obtained from dynamic programming equations, where $(o)$ and $(*)$ correspond to $(i-S)^{+}$and $\min (i, j)$ respectively 


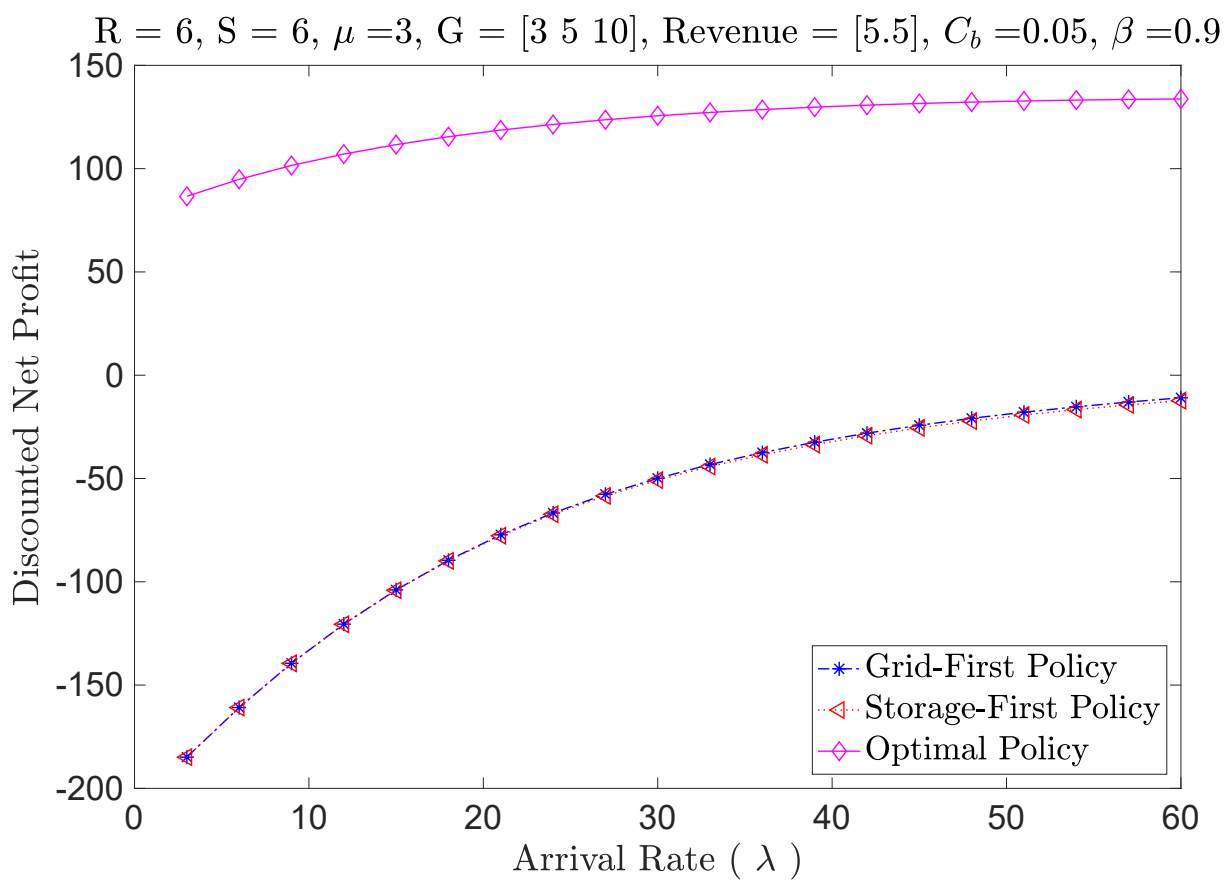

Figure 5.6: The Discounted Utility $\mathbf{U}(z)=-\mathbf{J}(z)$ per unit time for optimal policy is compared to heuristic policies "Grid-First" and "Storage-First" for different values of $\lambda$, where $V_{X}$ is constant.

- $g=g^{\prime}, j=j^{\prime}$ and $i^{\prime}>i$.

Finally, in Figures 5.6, 5.7, 5.8 and 5.9, the output of the optimal policy in Section 5.3 , is compared to two heuristic stationary policies for different values of $\lambda$. These heuristic policies are called "Grid-First" and "Storage-First" policies and defined and used in [80]. In both of these policies, $\alpha \equiv 1$ for all states. Also in both of them, new arrivals will be blocked, i.e. $\gamma=1$, only if there is not any available resource to serve the arriving demands; that is: $i_{X}=S+j_{X}$. The only difference is in splitting the customers between grid and the local storage unit; i.e. determining $i_{R}$. In Grid-First, customers will be served by the storage unit, only if all available $S$ power units from the grid are being used by other customers. Therefore: $i_{R}=(i-S)^{+}$. In contrast, in Storage-First policy, customers are by default served by the local storage unit, unless the number of demands is greater than the battery level $(i>j)$. So, in Storage-First policy, we have: $i_{R}=\min (i, j)$.

In these figures, the average Utility $\mathbf{U}$ per unit time is illustrated: $\mathbf{U}(z)=-\mathbf{J}(z)$ 


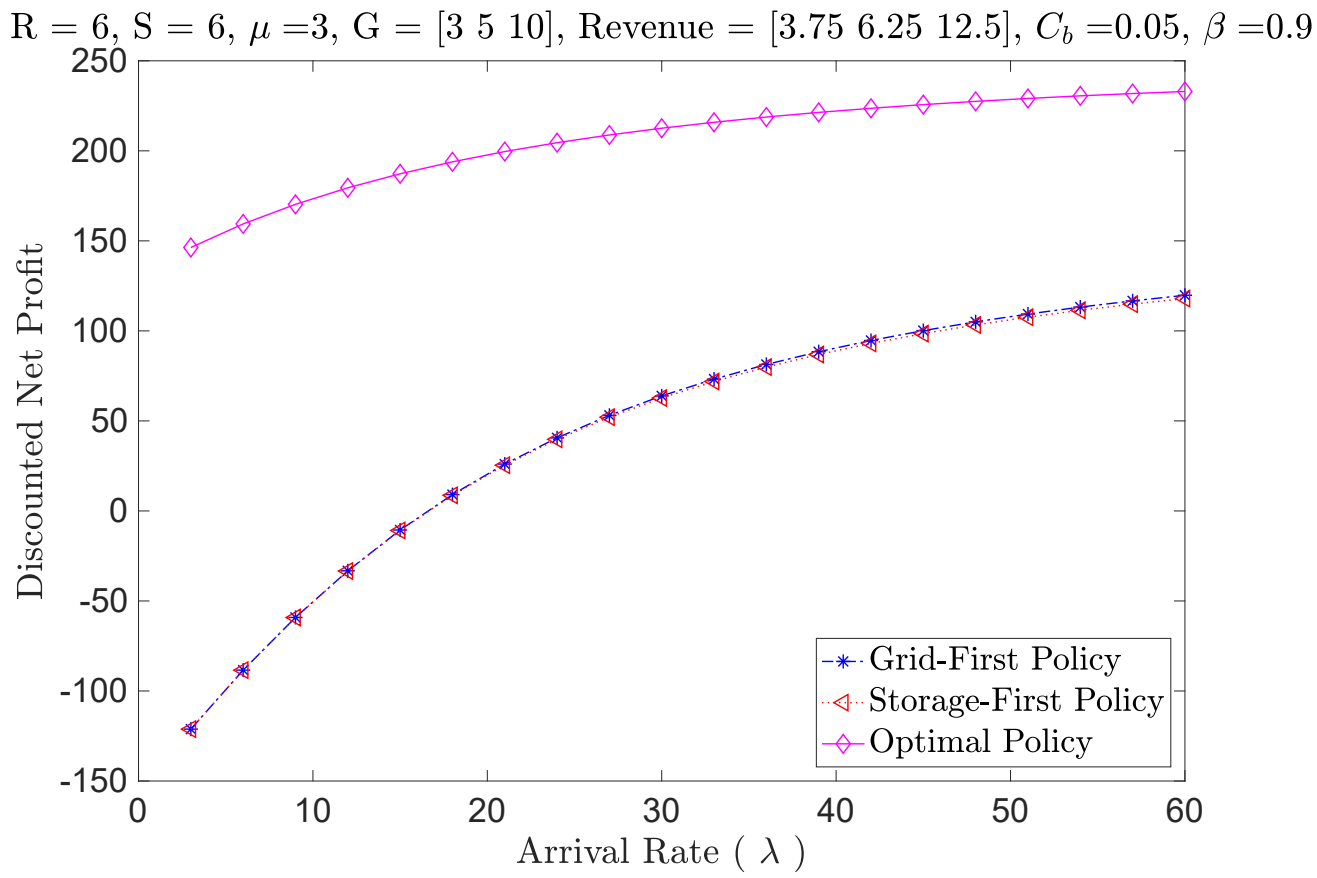

Figure 5.7: The Discounted Utility $\mathbf{U}(z)=-\mathbf{J}(z)$ per unit time for optimal policy is compared to heuristic policies "Grid-First" and "Storage-First" for different values of $\lambda$, where $V_{X}$ is variable.

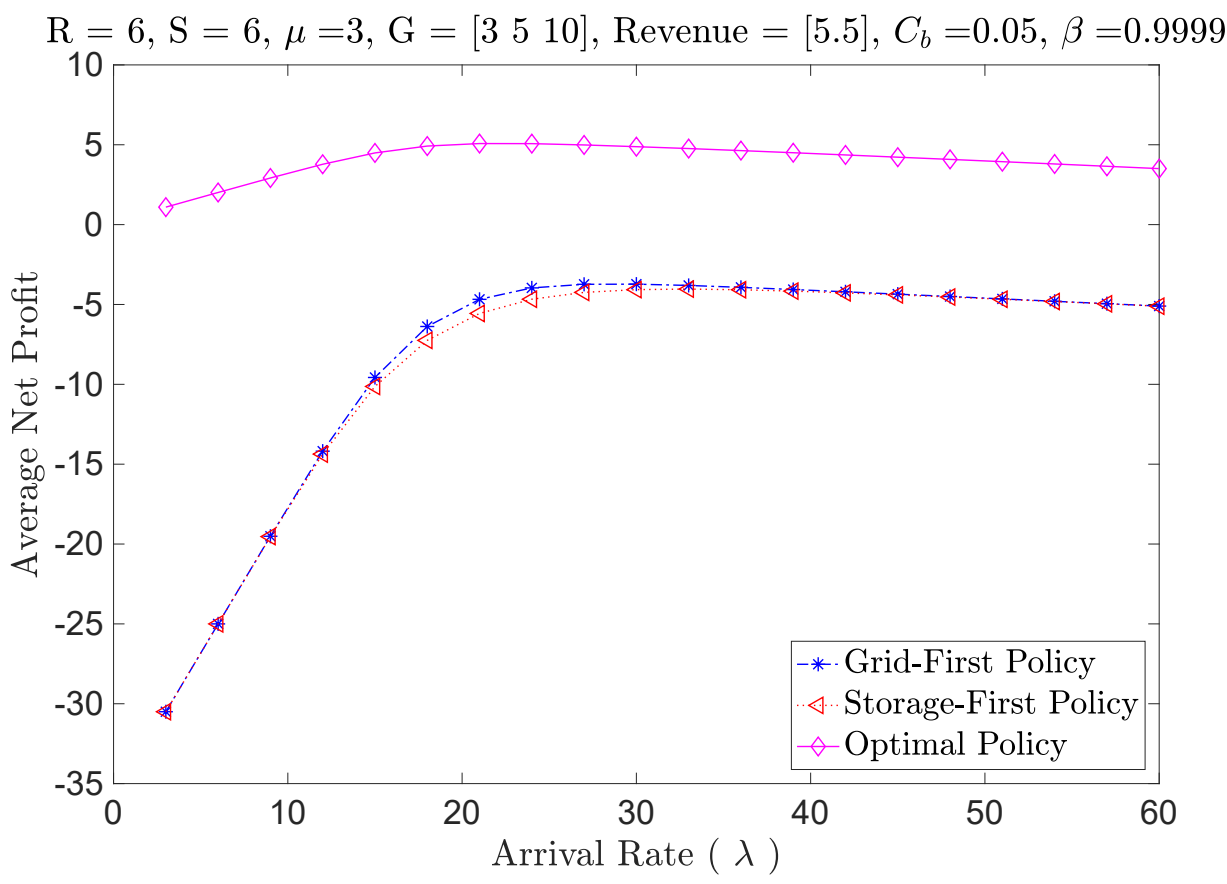

Figure 5.8: The average Utility $\mathbf{U}(z)=-\mathbf{J}(z)$ per unit time for optimal policy is compared to heuristic policies "Grid-First" and "Storage-First" for different values of $\lambda$, where $V_{X}$ is constant. 


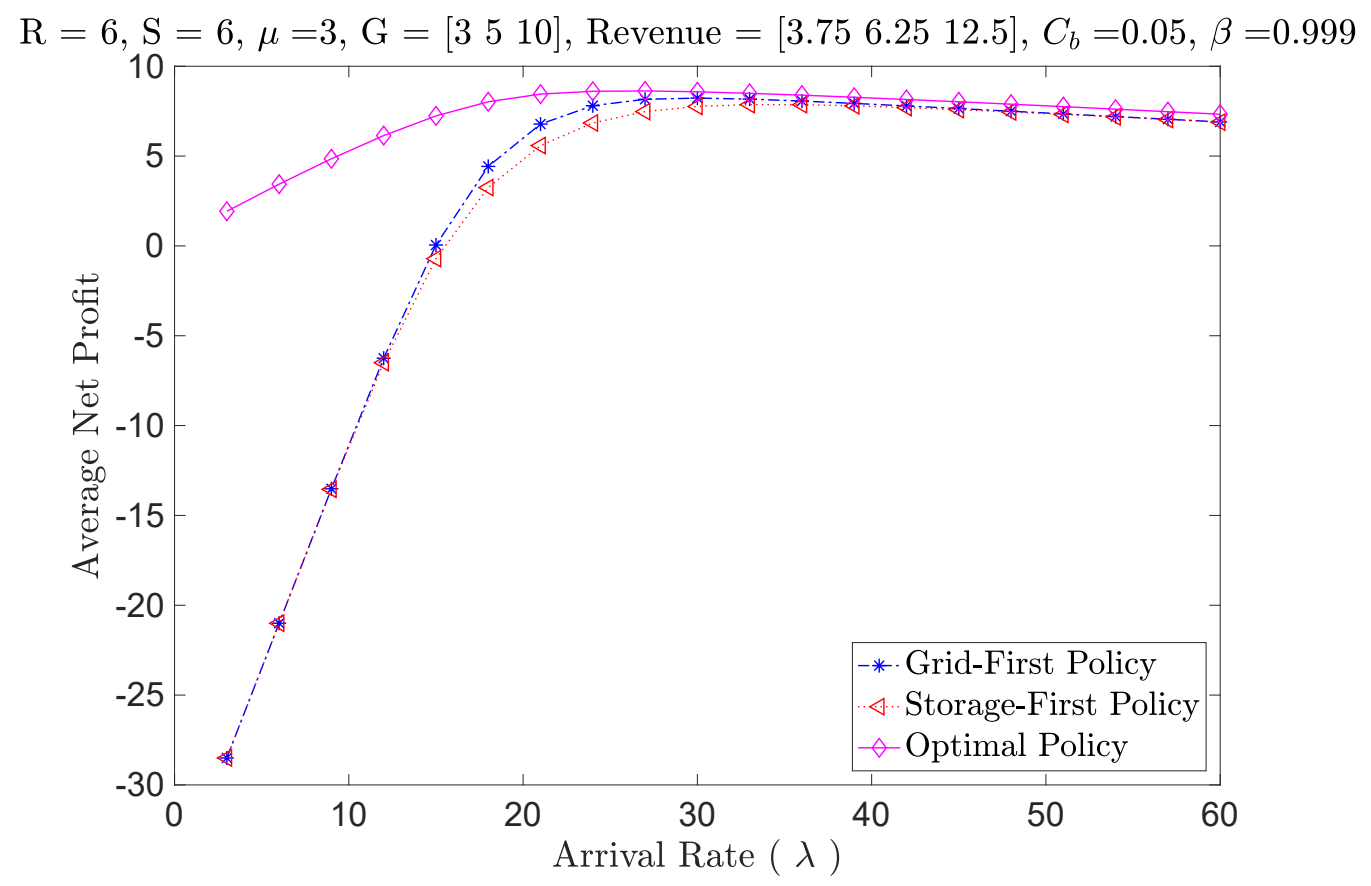

Figure 5.9: The average Utility $\mathbf{U}(z)=-\mathbf{J}(z)$ per unit time for optimal policy is compared to heuristic policies "Grid-First" and "Storage-First" for different values of $\lambda$, where $V_{X}$ is variable.

where $\mathbf{J}(z)$ is computed with respect to Equation (5.34) for each of these policies, including the optimal policy $z^{*}$. In these figures, the parameters of the charging station are almost the same as the ones given at the beginning of this Subsection. One exception is that to evaluate the expected $\beta$-discounted utility, in Figures 5.6 and 5.7, it is assumed that: $\beta=0.9$. Besides, in Figures 5.6 and 5.8, $V_{X}$, the revenue per unit time from each EV being charged, is assumed to be constant $V_{X} \equiv 5.5$. Whereas, in Figures 5.7 and 5.9, $V_{X}$ is assumed to be variable and is $0.25 \%$ more than $g$, the price of each unit of electrical power drawn from the grid at each moment, i.e. $V_{X}=1.25 \cdot g_{X}$.

In Figures 5.6 and 5.7, the expected total $\beta$-discounted cost/utility (Section 5.3.2) is evaluated. Since this cost depends on the initial state, in order to compare the output of different policies, we assume that the initial state is uniformly distributed in state space $\mathcal{X}$. Then, after computing the $\beta$-discounted expected cost $\mathbf{J}_{\mathbf{z}}^{\beta}(X)$ (Equation (5.29)), we perform the averaging based on this assumption. On the other hand, in Figures 5.8 and 5.9 , the long term average cost (Section 5.3.3) of the above policies are computed and compared with each other.

We can see in these figures, the output of the optimal policy $z^{*}$ dominates that 
of other policies, which is consistent with our analyses in this Section. In particular, these figures show that for lower arrival rates of EVs, we should expect bigger differences between the output of the optimal policy and that of the other two heuristic policies.

\subsection{Summary}

We considered the problem of optimally controlling a charging station of EVs. To isolate the peak demands from the electrical grid, we assumed that the amount of electrical power drawn from the grid is limited to a predetermined value. Then, to be able to better serve the stochastic charging demands, some local energy storage units are deployed in the station. They can be used to meet the excessive demands during rush hours and consequently smooth the total stochastic energy requirement profile of the station. On the other hand, if possible, any unused power from the grid will be used to charge these local storage units.

As a result, the charging station is modeled as continuous-time Markov Decision Process. In particular, the controller of the charging station should continuously decide about: whether to accept or block an arriving customer, how much of the energy demands should be met using the local storage units, and what should be the charging rate of each local storage unit. We proved the the existence of a stationary and bang-bang optimal policy minimizing the infinite horizon average cost. 


\section{Chapter 6}

\section{Optimal Controlling of Charging Stations for Heterogeneous Electric Vehicles}

In this chapter, we extend the problem of controlling a charging station of Electric Vehicles $(\mathrm{EVs})$ by considering different classes of EVs with respect to the type of the battery they are equipped with. In this case, the charging station will deploy a local storage unit for each class of customers. We start our analyses by presenting the resulted mathematical model, then we will show how to find the corresponding optimal policies for controlling the charging station. In particular, in addition to all control actions mentioned in the previous chapter, the controller has to decide how to split the available power from the grid between different classes of customers. We find optimal policies in two distinct scenarios: In the first scenario, the splitting decision is made off-line; that is before serving the customer starts. In the second scenario, we formulate and solve the optimization problem for Real-Time splitting of the available power from the grid between different classes of EVs, i.e. while serving the demands. We conclude the chapter by comparing the performance of the proposed policies with that of some known heuristic policies studied in the literature.

\subsection{Charging Station Model: Overall View}

In Chapter 5, we studied the problem of charging EVs, all equipped with the same type of battery. Here, we extend our analyses by taking into account different classes of EVs. We will conclude the chapter by presenting some numerical results.

Different types of EVs could deploy different types of batteries. Denote by $\mu$, the rate of charging each EV or one level of a storage unit. There are two main factors 
affecting the final charging rate of a storage unit or battery: The power rating $\left(S_{P R}\right)$ and the efficiency $(\eta)$. The latter one is the proportion of the stored energy over the total amount of energy used in charging the storage unit or battery. The power rating $\left(S_{P R}\right)$, is the maximum acceptable electrical power level to be used in charging the storage unit. If $\hat{S}$ units of power $\left(\hat{S} \leq S_{P R}\right)$ is used to charge the storage unit or battery, the final rate is equal to $\hat{S} \cdot \eta \cdot \mu$. In the sequel, for EVs, it is assumed that $S_{P R}=1$. In contrast, we assume that local storage units have higher power ratings $\left(S_{P R} \geq 1\right)$.

In our setting, EVs are classified with respect to their battery types, i.e. based on the average charging rate of their batteries. For simplicity in our notations, for each class of batteries, we merged the efficiency parameter $(\eta)$ into charging rate $\mu$ and

denote it by $\mu^{c}$, representing the charging rate of EVs in the $c^{t h}$ class of customers. For each class of EVs, we use a separate local storage unit to be used to serve the EVs of the same class. However, for the available power from the grid, it is the responsibility of the charging station operator to decide how to split this power between different classes of customers (Will be discussed later in more details). The rest of the model is the same as the one introduced in Chapter 5, Section 5.1. In the next section, we explain the model with mathematical details.

\subsection{Controlling the Charging Station with Multi- Class of customers}

Denote by $c \in\{1, \ldots, C\}$, the $c^{\text {th }}$ class of EVs. As we discussed in Section 6.1, different classes of EVs are differentiated based on their charging rate $\mu$ (Indeed, for each class of batteries, we merged the efficiency parameter $(\eta)$ into charging rate $\mu$ and denote the final result by $\mu^{c}$ ). So, the charging times of EVs of each class are i.i.d exponential random variables with rate $\mu^{c}$. In addition, customers of each class are arriving to the station according to a Poisson process with rate $\lambda^{c}$. Besides, the arrivals of EVs from different classes are assumed to be independent of each other.

The same as Sections 5.1 and 5.2, we assume that the station is drawing at most $S$ units of power from the grid. Besides, to better serve the customers, for each class of them, the charging station is equipped with a local storage unit of the same type. For example, if a station is serving two classes of fast charging and slow charging EVs, then it is deployed with two separate storage units of size $R^{1}$ and $R^{2}$. The available 


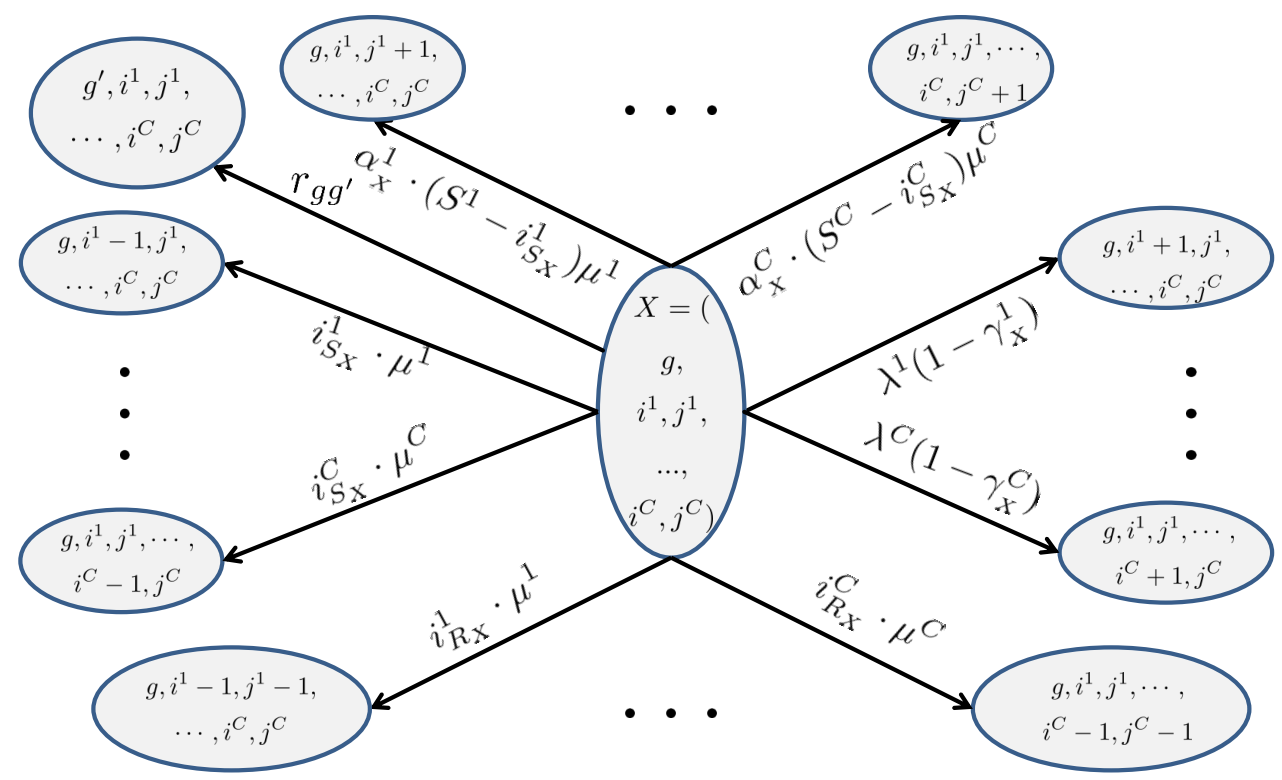

Figure 6.1: The out-flow of the state $X=\left(g, i^{1}, j^{1}, \ldots, i^{C}, j^{C}\right)$ in the charging station serving different classes of EVs

energy in each of these storage units is only being used by EVs of the same class. However, it is up to the controller how to split the available $S$ units of power from the grid between different classes of customers. Denote by $S^{c}$, the total units of power from the grid that the controller assigns to EVs of class $c$. We have: $S=\sum_{c=1}^{C} S^{c}$. Each of these $S^{c}$ units of electrical power are then used to either directly charge EVs of $c^{\text {th }}$ class or charge the $c^{\text {th }}$ storage unit at the station. The rest of our definitions and assumptions are similar to those presented in Sections 5.1 and 5.2, except that here we are dealing with multiple classes of customers.

The resulted problem represents a Markov Decision Process (MDP). Figure 6.1 illustrates the transition diagram in the resulted Markov process. As it is shown in this figure, the state of the charging station at each time $t \geq 0$ is determined by a $(2 C+1)$-dimensional vector $X=\left(g, i^{1}, j^{1}, \ldots, i^{C}, j^{C}\right)$, where $X \in \mathcal{X}$, and $\mathcal{X}$ is the state space of the resulted Markov Process. Following the definitions in Section 5.2, the state parameters, $g$ and $, i^{c}, j^{c}(c=1, \ldots, C)$ are defined as follows: $g$ denotes the price of each unit of electrical power in the grid at time $t . i^{c}$ denotes the number of EVs of class $c$ being served at the station and $j^{c}$ denotes the level of the storage unit dedicated to EVs of class $c$. Therefore, with respect to these definitions, at each time 
$t$ when the system is at state $X$, the conditions in Equations (5.1) and (5.2) become as follows (for clarity, we drop the indexes $X$ and $t$ from the parameters):

$$
\begin{gathered}
0 \leq j^{c} \leq R^{c} \quad \text { and } \quad 0 \leq i^{c} \leq S^{c}+j^{c} \\
0 \leq i_{S}{ }^{c} \leq S^{c} \quad \text { and } \quad 0 \leq i_{R}{ }^{c} \leq j^{c} \\
i_{R}{ }^{c}+i_{S}{ }^{c}=i^{c}
\end{gathered}
$$

Now, according to the definitions in this section, and the ones in Subsection 5.2.2, the control vector at each time $t$ and state $X$ is defined as a $(4 C)$-dimensional vector $\mathbf{u}_{X, t}=\left(S_{X, t}^{1}, \gamma_{X, t}^{1}, i_{R_{X, t}}^{1}, \alpha_{X, t}^{1}, \cdots, S_{X, t}^{C}, \gamma_{X, t}^{C}, i_{R_{X, t}}^{C}, \alpha_{X, t}^{C}\right)$. Control actions $\gamma_{X, t}^{c}, i_{R_{X, t}}^{c}, \alpha_{X, t}^{c}$ $(c=1, \ldots, C)$ are defined similar to their correspondents in Subsection 5.2.2. The control actions $S_{X, t}^{c}, c=1, \cdots, C$ are defined as the total units of power from the grid that the controller assigns to each class $c$, such that:

$$
\begin{gathered}
S=\sum_{c=1}^{C} S_{X, t}^{c} \\
S_{X, t}^{c} \geq 0, \quad c=1, \cdots, C
\end{gathered}
$$

Similar to Section 5.3, we define the instantaneous cost function of utilizing the charging station. Given the state at time $t$ is $X_{t}=\left(g, i^{1}, j^{1}, \ldots, i^{C}, j^{C}\right)$ and the selected action vector is $\mathbf{u}_{X, t}=\left(S_{X, t}^{1}, \gamma_{X, t}^{1}, i_{R_{X, t}}^{1}, \alpha_{X, t}^{1}, \cdots, S_{X, t}^{C}, \gamma_{X, t}^{C}, i_{R_{X, t}}^{C}, \alpha_{X, t}^{C}\right)$, the instantaneous cost function is defined as follows:

$$
\begin{aligned}
C_{t}\left(X, \mathbf{u}_{X, t}\right) d t & = \\
& \sum_{c=1}^{C}\left(g \cdot i_{S_{X, t}}{ }^{c}+g \cdot \alpha_{X, t}^{c} \cdot\left(S_{X, t}^{c}-i_{S_{X, t}}{ }^{c}\right)\right. \\
& \left.-V_{X} \cdot i^{c}+C_{b l}^{c} \cdot \lambda^{c} \cdot \gamma_{X, t}^{c}\right) d t
\end{aligned}
$$

This cost function is stationary and independent of the time. So, at each time $t$, we can write:

$$
C_{t}\left(X, \mathbf{u}_{X, t}\right) d t=C\left(X, \mathbf{u}_{X, t}\right) d t
$$

A control policy is a set of decision rules $\mathbf{z}=\left\{\mathbf{u}_{X, t}: \quad X \in \mathcal{X}, t\right\}$. Denote by $\mathcal{Z}$, the 
set of all policies. We are interested in finding a policy which minimizing the average incurred cost during the operating time of the system (Equations (5.33), (5.34) and (5.35)). To find the optimal policy, we again use the method of "uniformization" [74] and follow the same steps as the ones in Section 5.3. In particular, to find the transition probabilities, we should rewrite Equation 5.16 for the case of having multiple classes of EVs. The resulted transition probabilities are as follows:

$$
\begin{aligned}
& \mathbb{P}^{\mathbf{z}}\left(X_{k+1}=\left(g^{\prime}, \cdots, i^{\prime c}, j^{c}, \cdots\right) \mid\right. \\
& \left.X_{k}=\left(g, \cdots, i^{c}, j^{c}, \cdots\right), \mathbf{u}_{X, k}=\left(\cdots, S^{c}, \gamma^{c}, i_{R}^{c}, \alpha^{c}, \cdots\right)\right) \cdot \rho \\
& = \begin{cases}r_{g g^{\prime}} & g^{\prime} \neq g \\
\cdot & i^{\prime}=i^{c}+1 \\
\lambda^{c}\left(1-\gamma^{c}\right) \cdot 1_{\left\{i^{c}<S^{c}+j^{c}\right\}} & i^{c}=i^{c}-1, j^{c}=j^{c}-1 \\
i_{S}^{c} \mu^{c} 1_{\left\{i^{c} j^{c}>0\right\}} \mu^{c} 1_{\left\{i^{c}>0\right\}} & i^{c}=i^{c}-1 \\
\alpha^{c}\left(S^{c}-i_{S}^{c}\right) \mu^{c} \cdot 1_{\left\{j^{c}<R^{c}\right\}} & i^{c}=i^{c}+1 \\
\cdot & \\
\cdot & \\
\cdot & X_{k+1}=X_{k} \\
\rho-\rho^{\prime} & \end{cases}
\end{aligned}
$$

Where

$$
\begin{aligned}
& \rho^{\prime}=\sum_{g^{\prime} \neq g} r_{g g^{\prime}} \\
& +\sum_{c=1}^{C} \quad\left(\lambda^{c}\left(1-\gamma^{c}\right) \cdot 1_{\left\{i^{c}<S^{c}+j^{c}\right\}}+i_{R}^{c} \mu^{c} 1_{\left\{i^{c} j^{c}>0\right\}}\right. \\
& \left.\quad+i_{S}^{c} \mu^{c} 1_{\left\{i^{c}>0\right\}}+\alpha^{c}\left(S^{c}-i_{S}^{c}\right) \mu^{c} \cdot 1_{\left\{j^{c}<R^{c}\right\}}\right)
\end{aligned}
$$

The optimal policy is obtained from the discrete time formulation of the dynamic programming equation (Equation (5.19)). Expanding Equation (5.19) for multi-class 
of EVs, results in the following equation (For simplicity in our notations, in the following equations, we replace $\left(S_{X}^{c}, \gamma_{X}^{c}, i_{R_{X}}^{c}, \alpha_{X}^{c}\right)$ with $\left.\left(S^{c}, \gamma^{c}, i_{R}{ }^{c}, \alpha^{c}\right)\right)$ :

$$
\begin{aligned}
& \mathbf{J}_{k+1}^{\beta}(X)=\min _{\mathbb{S}}\left\{\sum_{c=1}^{C}(\right. \\
& \min _{\gamma^{c} \in[0,1]}\left\{\lambda ^ { c } \gamma ^ { c } \left(C_{b l}^{c}-\frac{\beta}{\rho} 1_{\left\{i^{c}<S^{c}+j^{c}\right\}}\right.\right. \\
& \left.\left.\left(\mathbf{J}_{k}^{\beta}\left(g, \cdots, i^{c}+1, j^{c}, \cdots\right)-\mathbf{J}_{k}^{\beta}\left(g, \cdots, i^{c}, j^{c}, \cdots\right)\right)\right)\right\} \\
& +\min _{i_{R}^{c} \in\left\{\left(i^{c}-S^{c}\right)^{+}, \cdots, \min \left(i^{c}, j^{c}\right)\right\}, \alpha^{c} \in[0,1]}\{ \\
& i_{S}^{c}\left(g+\frac{\beta \mu^{c}}{\rho} 1_{\left\{i^{c}>0\right\}}\right. \\
& \left(\mathbf{J}_{k}^{\beta}\left(g, \cdots, i^{c}-1, j^{c}, \cdots\right)-\mathbf{J}_{k}^{\beta}\left(g, \cdots, i^{c}, j^{c}, \cdots\right)\right) \\
& +i_{R}^{c} \frac{\beta \mu^{c}}{\rho} 1_{\left\{i^{c} j^{c}>0\right\}} \\
& \left(\mathbf{J}_{k}^{\beta}\left(g, \cdots, i^{c}-1, j^{c}-1, \cdots\right)-\mathbf{J}_{k}^{\beta}\left(g, \cdots, i^{c}, j^{c}, \cdots\right)\right) \\
& +\alpha^{c}\left(S^{c}-i_{S}^{c}\right)\left(g+\frac{\beta \mu^{c}}{\rho} 1_{\left\{j^{c}<R^{c}\right\}}\right. \\
& \left.\left.\left.\left.\left(\mathbf{J}_{k}^{\beta}\left(g, \cdots, i^{c}, j^{c}+1, \cdots\right)-\mathbf{J}_{k}^{\beta}\left(g, \cdots, i^{c}, j^{c}, \cdots\right)\right)\right)\right\}\right)\right\} \\
& + \text { terms not depending on }\left(S^{c}, \gamma^{c}, i_{R}^{c}, \alpha^{c}\right)
\end{aligned}
$$

where $\mathbb{S}=\left(S^{1}, \cdots, S^{C}\right)$ is a $C$-dimensional vector with its elements are the chosen values of $S^{c}, c \in\{1, \cdots, C\}$. Equation (6.7) shows that for a given vector $\mathbb{S}$, the optimal actions $\gamma^{c}, \alpha^{c}$ and $i_{R}^{c}$ are obtained with respect to the equations similar to the ones derived in Section 5.3 for single class of customers, i.e. the Criteria in (5.21), (5.22), (5.23) and (5.27). In the sequel, we show how to find the optimal policies in two different scenarios with respect to splitting the power from the grid between different classes of customers.

\subsubsection{Off-line splitting of Power from the Grid}

In the first scenario, we assume that the values of $S^{c}, c \in\{1, \cdots, C\}$ are determined before serving the EVs starts. This scenario is the same as the one, studied in [80], in order to compare the performance of heuristic policies "Grid-First" and "StorageFirst" (Section 5.3.4). However, in [80], $S^{c} \mathrm{~s}, c=1, \cdots, C$ were the only control 
actions determined by the controller. In this scenario, all $S^{c}$ s remain the same for all the states and during the whole time horizon of deploying the charging policy. That is, if we denote by a $C$-dimensional vector $\mathbb{S}_{X, t}=\left(S^{1}, \cdots, S^{C}\right)$, the values of control action $S^{c}, c=1, \cdots, C$ at time $t$ and state $X$, then, we have: $\mathbb{S} \equiv \mathbb{S}_{X, t}$ for all $t \geq 0$ and $X \in \mathcal{X}$.

The resulting optimization problem is as follows (For simplicity in notations, we dropped the index $t$ ):

$$
\begin{gathered}
\arg \min _{\mathbf{z}} \mathbf{J}(\mathbf{z}) \\
S^{c} \geq 0, \\
S=\sum_{c=1}^{C} S^{c} \\
0 \leq \gamma_{X}^{c} \leq 1 \\
0 \leq \alpha_{X}^{c} \leq 1 \\
\max \left(0, i_{X}^{c}-S^{c}\right) \leq i_{R_{X, c}} \leq \min \left(i_{X}^{c}, j_{X}^{c}\right) \\
c=1, \cdots, C
\end{gathered}
$$

Where $\mathbf{J}(\mathbf{z})$ is the average cost of deploying the policy $\mathbf{z}$ per unit time and defined in Equation (5.34).

To find the solution of this optimization problem, we first find all possible values of the $C$-dimensional vector $\mathbb{S}=\left(S^{1}, \cdots, S^{C}\right)$. Then for each of these vectors, let say $\mathbb{S}_{1}$, we solve the dynamic programming Equation (6.7), using the the Criteria in (5.21), (5.22), (5.23) and (5.27) to find the rest of control actions $\alpha_{X}^{c}, i_{R_{X}}^{c}, \gamma_{X}^{c}$ for all $c \in\{1, \cdots, C\}$ and $X \in \mathcal{X}$. The total number of states, i.e. the size of $\mathcal{X}$, for each choice of $\mathbb{S}=\left(S^{1}, \cdots, S^{C}\right)$ is calculated as follows:

$$
|\mathcal{X}|=\sum_{g \in G}\left(\sum_{j^{1}=0}^{R^{1}} \sum_{i^{1}=0}^{S^{1}+j^{1}} \cdots \sum_{j^{C}=0}^{R^{C}} \sum_{i^{C}=0}^{S^{C}+j^{C}} 1\right)
$$

Eventually, for each vector $\mathbb{S}$, like $\mathbb{S}_{1}$, we find the corresponding optimal policy $\mathbf{z}_{1}^{*}$, resulting in $\mathbf{J}\left(\mathbf{z}_{\mathbf{1}}^{*}\right)$. Then, the ultimate optimal policy, $\mathbf{z}^{*}$, is the one resulting in the minimum average cost among all of them. 


\subsubsection{Real-Time splitting of Power from the Grid}

In the previous subsection (as well as [80]), the assumption was that the splitting the power from the grid between different classes of customers happens before serving the EVs starts and remains the same through-out the service. In this subsection, we go one step further and let the splitting decision by the controller occur in "Real-Time", while serving the customers. As a result, at time $t$ when the station is at state $X \in \mathcal{X}$, the controller has to determine all the control actions $\left(S_{X, t}^{1}, \gamma_{X, t}^{1}, i_{R_{X, t}}^{1}, \alpha_{X, t}^{1}, \cdots, S_{X, t}^{C}, \gamma_{X, t}^{C}, i_{R_{X, t}}^{C}, \alpha_{X, t}^{C}\right)$ together, including the $C$-dimensional vector $\mathbb{S}_{X, t}=\left(S_{X, t}^{1}, \cdots, S_{X, t}^{C}\right)$.

The optimization problem for "Real-Time" scenario is as follows (For simplicity in notations, we dropped the index $t$ ):

$$
\begin{array}{ll} 
& \arg \min _{\mathbf{z}} \mathbf{J}(\mathbf{z}) \\
\text { s.t. } \quad & S_{X}^{c} \geq\left(i_{X}^{c}-j_{X}^{c}\right)^{+}, \\
& S=\sum_{c=1}^{C} S_{X}^{c} \\
0 & \leq \gamma_{X}^{c} \leq 1 \\
0 & \leq \alpha_{X}^{c} \leq 1 \\
\max \left(0, i_{X}^{c}-S_{X}^{c}\right) & \leq i_{R_{X, c}} \leq \min \left(i_{X}^{c}, j_{X}^{c}\right) \\
c & =1, \cdots, C
\end{array}
$$

Where $\mathbf{J}(\mathbf{z})$ is the average cost of deploying the policy $\mathbf{z}$ per unit time and defined in Equation (5.34). Compared to "Off-line splitting" scenario, (Equation (6.8)), here we have a new constraint on each $S^{c}$, that is: $S^{c} \geq\left(i^{c}-j^{c}\right)^{+}$, where $\left(i^{c}-j^{c}\right)^{+}=$ $\max \left(0, i^{c}-j^{c}\right)$. This new constraint assures us that at each state any EV being served at the station will continue to receive the service.

To find the solution of the optimization problem (6.10), for each state $X \in \mathcal{X}$, we first find all possible values of the $C$-dimensional vector $\mathbb{S}=\left(S^{1}, \cdots, S^{C}\right)$, with 
respect to the following constraints:

$$
\begin{aligned}
& S^{c} \geq\left(i^{c}-j^{c}\right)^{+}, \quad c \in\{1, \cdots, C\} \\
& S=\sum_{c=1}^{C} S^{c}
\end{aligned}
$$

Then, for each of these vectors, let say $\mathbb{S}_{1}$, we solve the dynamic programming Equation (6.7), using the the Criteria in (5.21), (5.22), (5.23) and (5.27) to find the rest of control actions $\alpha_{X}^{c}, i_{R_{X}}^{c}, \gamma_{X}^{c}$ for that state and for all $c \in\{1, \cdots, C\}$. Then, the ultimate optimal actions for the state $X$ are the ones resulting in the minimum average cost among all of them. We do this for all states $X \in \mathcal{X}$ and for all iteration of solving the dynamic programming Equation (6.7). The total number of states, i.e. the size of $\mathcal{X}$, in "Real-Time" scenario, is calculated as follows:

$$
\begin{aligned}
& |\mathcal{X}|=\sum_{g \in G}\left(\sum_{j^{1}=0}^{R^{1}} \cdots \sum_{j^{C}=0}^{R^{C}} \sum_{i^{1}=0}^{S^{1}+j^{1}}\right. \\
& \left.\sum_{i^{2}=0}^{S^{2}+j^{2}+\left(j^{1}-i^{1}\right)^{+}} \cdots \sum_{i^{C}=0}^{S^{C}+j^{C}+\left(j^{1}-i^{1}\right)^{+}+\cdots+\left(j^{C-1}-i^{C-1}\right)^{+}} 1\right)
\end{aligned}
$$

\subsubsection{Numerical Examples}

In this Section, we examine the Optimal Policies of controlling a charging station serving different classes of EVs. In our experiments, we assume that there are two classes of EVs, slow charging $(c=1)$ vs. fast-charging $(c=2=C)$, where the charging rate of fast-charging "EVs" (and also their corresponding local storage unit) is twice that of the slow-charging EVs, i.e. $\mu^{2}=2 \mu^{1}$. We assume that there are $S=6$ units of available power from the grid. The two deployed local storage units (each for each class) are with the same capacities, $R^{1}=R^{2}=3$. The arrival rates of both classes are assumed to be the same, i.e. $\lambda^{1}=\lambda^{2}=\frac{\lambda}{2}$, where $\lambda$ is the total arrival rate of EVs to the station, that is: $\lambda=\lambda^{1}+\lambda^{2}$. The blocking costs of EVs are assumed to be different for each class of EVs: $C_{b}^{1}=0.05$ and $C_{b}^{2}=0.1$.

The rest of the parameters are as follows: The given power unit price set is: $G=\{3,5,10\}$. All of these unit prices are assumed to have the same mean times, e.g. 


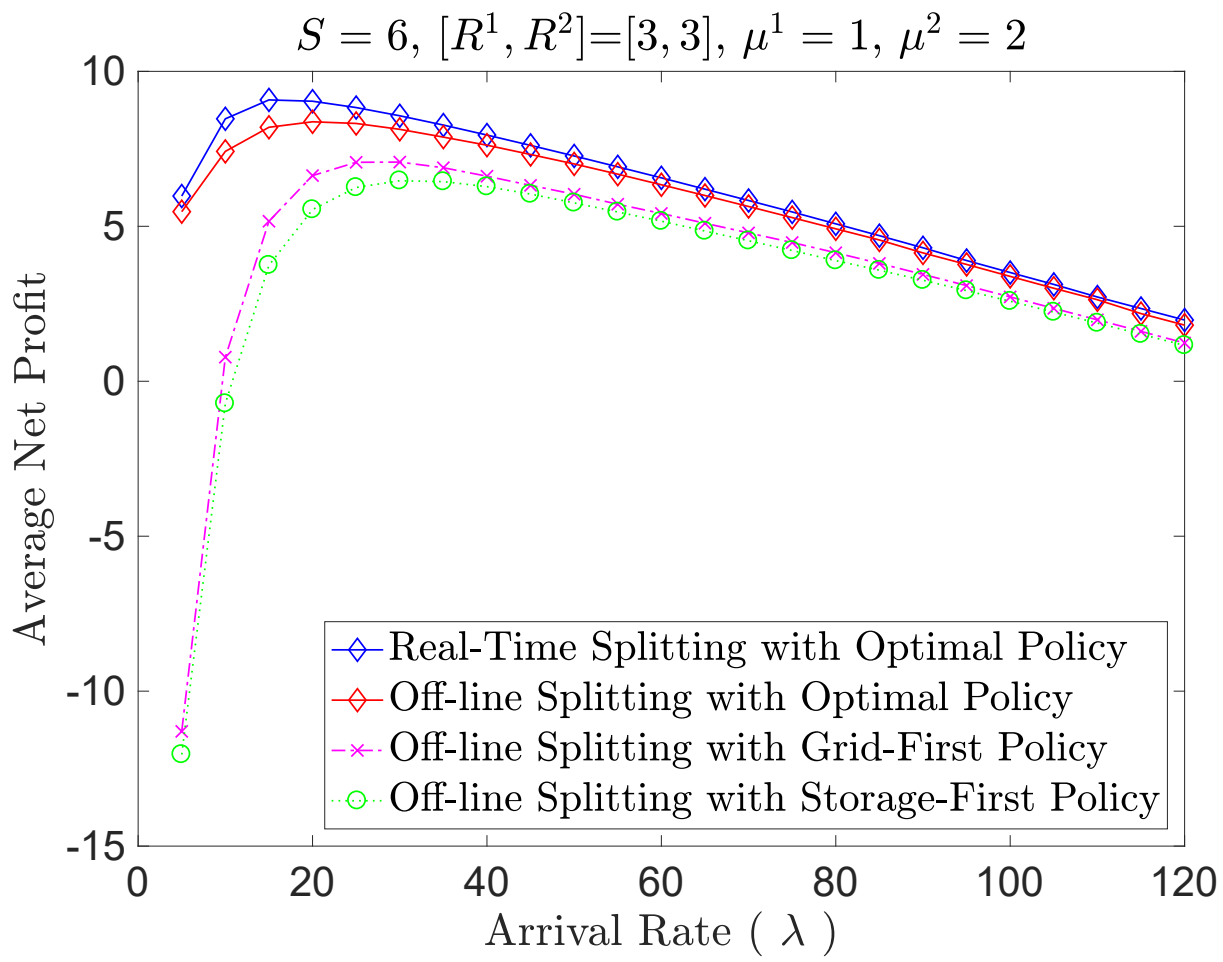

Figure 6.2: The average Utility $\mathbf{U}(z)=-\mathbf{J}(z)$ per unit time for Real-Time and Offline splitting of Power from the Grid optimal policies are compared to heuristic policies "Grid-First" and "Storage-First" for different values of $\lambda$, where $\mu^{1}=1$ and $\mu^{2}=2$.

8 hours in a day, and equal transition probabilities to all other unit prices, i.e. $\frac{1}{2}$ in our setting. Therefore, the transition rate between different unit prices are as follows: $r_{g g^{\prime}}=\frac{1}{16}$ for all $g \neq g^{\prime}$. The parameter, $V_{X}$, the revenue obtained by the charging station per unit time from each EV being charged at the station is assumed to be variable and is $0.25 \%$ more than $g$ (the price of each unit of electrical power drawn from the grid at each moment, i.e. $\left.V_{X}=1.25 \cdot g_{X}\right)$. Eventually. in our analyses, we assumed that: $\beta=0.9999$. The same as Section 5.3.4, in all figures in this section, the average Utility $\mathbf{U}$ per unit time is illustrated: $\mathbf{U}(z)=-\mathbf{J}(z)$ where $\mathbf{J}(z)$ is computed with respect to Equation (5.34) for each of these policies.

Figures 6.2 and 6.3 illustrate the the average Utilities of the optimal policies introduced in Section 6.2 for different values of $\lambda$. In Figure 6.2, we assumed $\mu^{1}=1$, while in Figure 6.3, $\mu^{1}=5$. Finally, in Figure 6.4, the average Utilities are computed and compared for different total arrival rates, $\lambda$, where the charging rate of slowcharging EVs vary from 1 to 5. In all of these figures, we compare the the average 


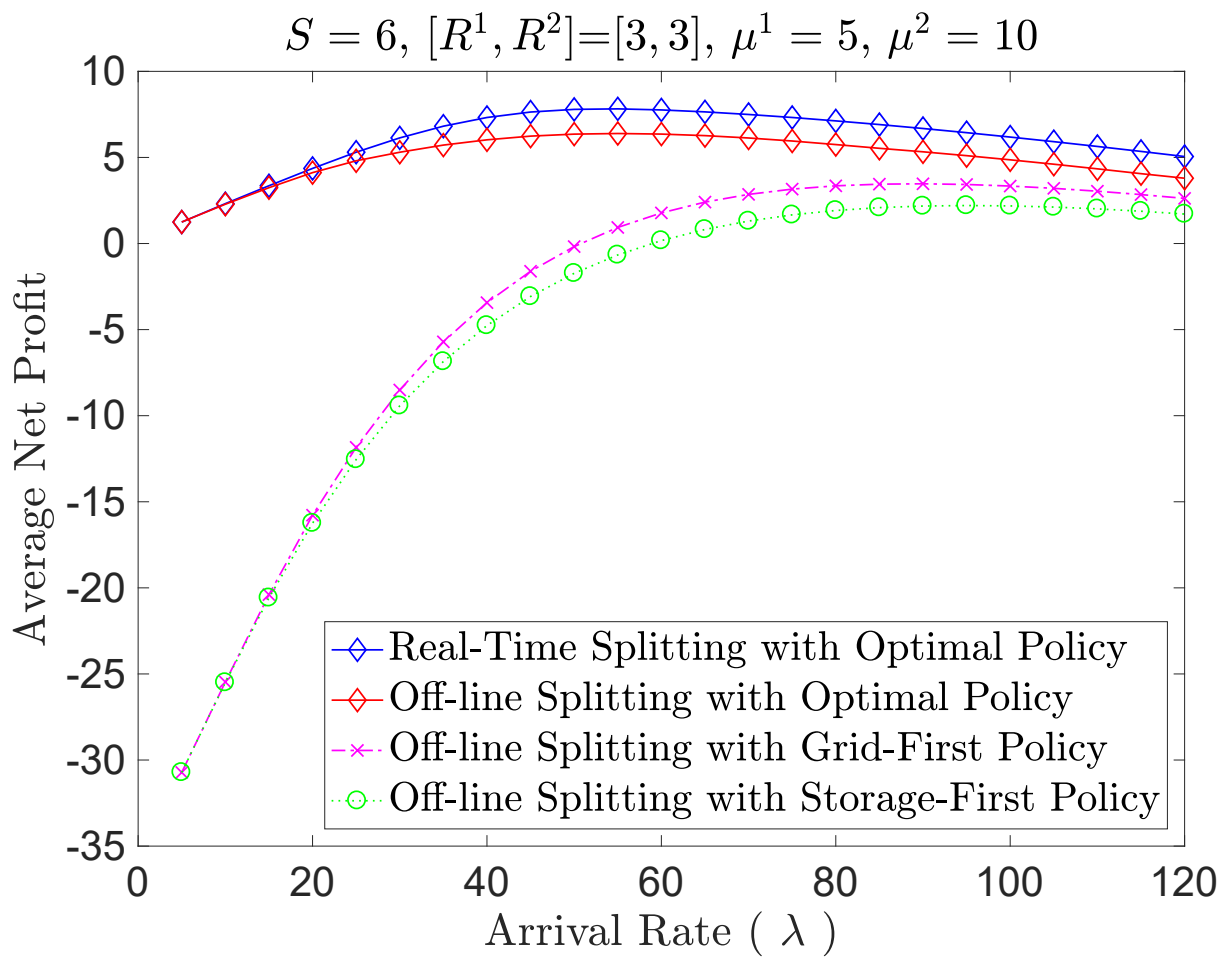

Figure 6.3: The average Utility $\mathbf{U}(z)=-\mathbf{J}(z)$ per unit time for Real-Time and Offline splitting of Power from the Grid optimal policies are compared to heuristic policies "Grid-First" and "Storage-First" for different values of $\lambda$, where $\mu^{1}=5$ and $\mu^{2}=10$.

Utilities of the optimal policies with those of in heuristic policies: "Grid-First" and "Storage-First" defined and used in [80] (Section 5.3.4).

As we expected from our analyses, the outputs of the optimal policies dominate that of other policies. Especially, "Real-Time" splitting with optimal policy (Optimization Problem (6.10)) shows the best performance. Also, according to Figures 6.2 and 6.3, the difference between the utility of the optimal and the heuristic policies becomes bigger for lower total arrival rates of customers. Moreover, as we can see in Figure 6.4, this difference grows as the charging rates of EVs grow. It means that for relatively higher charging rates of EVs, the optimal policies return better outcomes compared to that of the heuristic policies.

\subsection{Summary}

We considered the problem of optimally controlling a charging station serving multiple classes of EVs. We assumed that the charging station dedicates a distinct local 


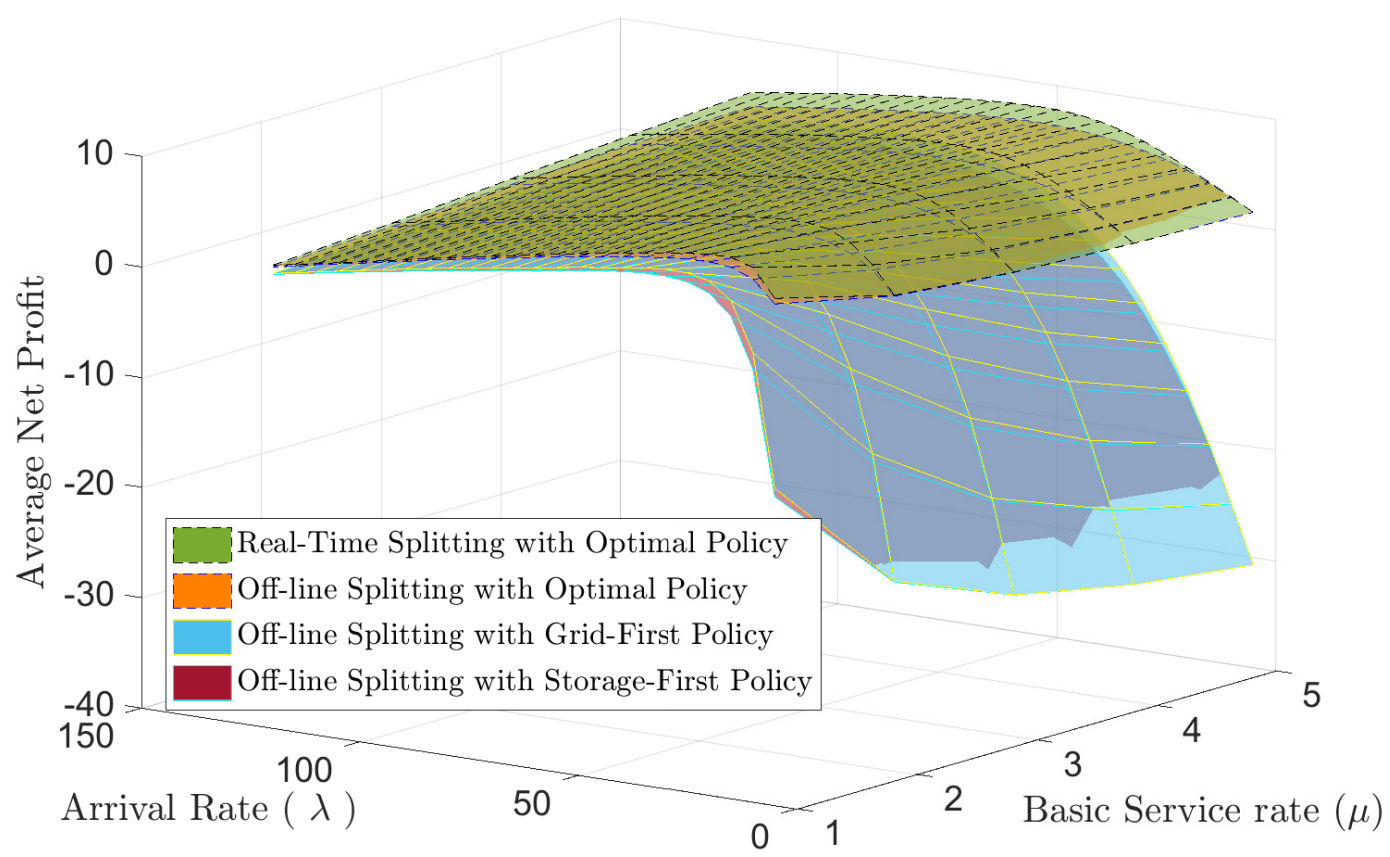

Figure 6.4: The average Utility $\mathbf{U}(z)=-\mathbf{J}(z)$ per unit time for Real-Time and Offline splitting of Power from the Grid optimal policies are compared to heuristic policies "Grid-First" and "Storage-First" for different values of $\lambda$, where $\mu^{1}=$ $[1,2,3,4,5]$ and $\mu^{2}=2 \mu^{1}$.

storage unit to each class of customers. Similar to the single-class scenario studied in Chapter 5, the charging station is modeled as continuous-time Markov Decision Process. In particular, a controller of a charging station should continuously decide about: whether to accept or block an arriving customer, how much of the energy demands should be met using the local storage units, and what should be the charging rate of each local storage unit. In addition to these control action, the controller has to decide how to split the available power from the grid between different classes of EVs. We first derived the resulted optimization problem for the cases where splitting decision is made offline, i.e. before serving the EVs starts. Then, we went further and derived and solved the optimization problem for real-time splitting of the available power form the grid; that is splitting decisions are made continuously while serving the customers. We concluded the chapter by presenting some numerical results supporting our analyses. In particular, we compared the performance of the proposed policies with some well-known heuristic policies. 


\section{Chapter 7}

\section{Conclusions and Suggestions for Future Research}

\subsection{Conclusions}

In this thesis, we studied the problem of optimal scheduling of flexible electric energy demands in a smart-grid framework. First inspired by the existence of electric appliances with flexibility on their charging rate, we introduced a model for the demand side of an electrical grid, as a set of "Malleable Rectangular-Shape" energy requirements. By "rectangular-shape" demand, we mean each energy requirement requires a certain amount of electrical energy, represented as the rectangle's area, and has to be supplied without interruption in a finite service interval with a constant power intensity. All of these energy requirements need to be served it a finite time interval, e.g. $[0,1]$. In addition, each demand is "Malleable"; that is, it has to be served with possible service time duration between a minimum and a maximum malleability constraints, which are given system parameters. Then a controller has to determine the starting time and the service duration for each and every demand, while service durations should satisfy malleability constraints. The set of all these starting times and the service durations are called a "scheduling policy". At each moment of time, the total energy consumption rate, or equivalently the total power of the grid is the sum of all consumption rates of the demands being supplied at that moment.

In Chapter 3, we only considered fixed malleability constraints, where malleability constraint pairs are the same for all demands. We first identified the lower bound on the optimal value of the power peak for all possible malleable constraint pairs. Then we introduced a linear time asymptotically optimal algorithm that is its asymptotic 
worst case performance ratio is equal to 1, i.e. the power peak will almost surely converge to its lower bound, when the number of demand is high. Then in Chapter 4, we extended our study of scheduling malleable electric demands, by letting each demand have its own malleability constraints and also by taking into account a convex cost of power consumption in our evaluations. Moreover, we considered an asymptotic setting with a large number of relatively small energy demands. We started our analyses by presenting an asymptotic analysis of stochastic demands to find the proper tight lower bounds for two types of costs in the system, i.e. power peak and total convex cost. Eventually we proposed an on-line scheduling algorithm for demands with stochastic energy demands and stochastic malleability constraints and showed that the presented algorithm is asymptotically optimal and also has fully linear running time.

In Chapter 5, we studied the problem of serving PHEVs in a charging station. Here PHEVs were considered as flexible demands due to the assumption that they can tolerate rejection of their energy request. In our model for the charging station, we assumed that it is equipped with a local storage energy unit with finite capacity. In particular, we included a Real-Time Pricing scheme in our treatment, in order to address the price fluctuations for electrical power in a grid. Our goal was to find a control policy minimizing the operational cost of the charging station, while considering the stability of the grid and Quality of Service. So we first modelled the charging station as a continuous time Markov Decision Process with three control actions at each state: whether to accept or block the arriving demands, determining the charging rate of the local storage unit and deciding on the number of demands being served by the storage unit. Using this model, we were able to use Dynamic Programming Techniques, which are widely used in Network Optimization problems. We proved that a stationary and bang-bang optimal policy exists, which minimizes a discounted cost over an infinite time horizon. Then we extended our analyses to find an optimal policy minimizing the infinite horizon average cost.

Eventually, in Chapter 6, we considered the problem of optimally controlling a charging station serving multiple classes of EVs. We assumed that the charging station dedicates a distinct local storage unit to each class of customers. Similar to the single-class scenario studied in Chapter 5, the charging station is modeled as continuous-time Markov Decision Process. In particular, a controller of a charging station should continuously decide about: whether to accept or block an arriving 
customer, how much of the energy demands should be met using the local storage units, and what should be the charging rate of each local storage unit. In addition to these control action, the controller has to decide how to split the available power from the grid between different classes of EVs. We first derived the resulted optimization problem for the cases where splitting decision is made offline, i.e. before serving the EVs starts. Then, we went further and derived and solved the optimization problem for real-time splitting of the available power form the grid; that is splitting decisions are made continuously while serving the customers.

\section{$7.2 \quad$ Future Directions}

Here are some possible directions we are suggesting for the future:

- In Chapter 3, for any possible fixed malleability constraint pair, we introduced an asymptotically optimal policy minimizing the power peak. As a future study, someone could try to find asymptotically optimal policies with respect to the total convex of the power consumption for cases where full coverage of the scheduling interval $[0,1]$ is not possible.

- In Chapter 4, we introduced an optimal policy minimizing the cost of serving malleable rectangular-shape demands, where malleability constraint pairs where identically and independently distributed random variable based on some general distribution. A possible direction would be finding a policy minimizing the convex cost of serving the demands with deterministic malleability constraint pair.

- In Chapters 5 and 6 , we studied the problem of minimizing the cost of serving EVs in a charging station. As a possible future direction, we may consider how to mathematically characterize the switching curve separating different regions corresponding to each particular action in the optimal policy.

- As another extension to optimal deployment of charging stations, we may consider some different models for representing the charging times of EVs, as well as the local storage unit. As examples, we could mention deterministic or some bounded distributions to model the charging time of EVs. 
- In the optimization problems studied in Chapters 5 and 6 , we did not address the problem in the presence of some fault or malfunction in the station. For example, someone could take into account:

- the probability that any of the charging stands becomes out of service (either while it is serving a customer or it is idle).

- The probability that the local storage unit becomes partially or completely unresponsive.

- The probability of an outage in the grid, such that the expected power from the grid becomes unavailable.

- In the proposed models for the charging station, we did not consider any queue; that is in cases where all available power is used to serve existing customers, any new customer will be blocked. A possible direction will be considering either a finite or infinite length queue and take into account its effect in calculating the cost of deploying the charging station and consequently finding the optimal policy.

- Instead of the Real-Time Pricing scheme considered in Chapters 5 and 6, someone could study the problem under some deterministic pricing schemes, like double-tariff schemes, such that the price of each unit of electrical power changes only at some known points during a day. 


\section{List of References}

[1] S. Massoud Amin and B. F. Wollenberg, "Toward a smart grid: power delivery for the 21st century," Power and Energy Magazine, IEEE, vol. 3, no. 5, pp. 34-41, 2005 .

[2] R. H. Lasseter, "Microgrids," in Power Engineering Society Winter Meeting, 2002. IEEE, vol. 1, pp. 305-308, IEEE, 2002.

[3] H. Farhangi, "The path of the smart grid," Power and Energy Magazine, IEEE, vol. 8, no. 1, pp. 18-28, 2010.

[4] R. G. Pratt, P. Balducci, C. Gerkensmeyer, S. Katipamula, M. C. Kintner-Meyer, T. F. Sanquist, K. P. Schneider, and T. Secrets, The Smart Grid: An Estimation of the Energy and CO2 Benefits. Pacific Northwest National Laboratory, 2010.

[5] M. Erol-Kantarci and H. T. Mouftah, "The impact of smart grid residential energy management schemes on the carbon footprint of the household electricity consumption," in IEEE Electrical Power and Energy Conference, 2010.

[6] A. Ipakchi and F. Albuyeh, "Grid of the future," IEEE Power and Energy Magazine, vol. 7 , Issue 2, pp. 52-62, 2009.

[7] L. D. Kannberg, M. C. Kintner-Meyer, D. P. Chassin, R. G. Pratt, L. A. DeSteese, J. G.and Schienbein, S. G. Hauser, and W. M. Warwick, GridWise: The Benefits of a Transformed Energy System. Pacific Northwest National Laboratory under contract with the United States Department of Energy. http://arxiv.org/pdf/nlin/0409035, 2003.

[8] T. e. t. s. o. o. F. RTE, "Daily load curve of the power consumption in france power distribution network on february 4, 2016.." http://clients. rte-france.com/lang/an/visiteurs/vie/courbes. jsp, 2016.

[9] L. D. Kannberg, D. P. Chassin, J. G. DeSteese, S. G. Hauser, M. C. KintnerMeyer, R. G. Pratt, L. A. Schienbein, and W. M. Warwick, "Gridwisetm: The benefits of a transformed energy system," arXiv preprint nlin/0409035, 2004.

[10] L. Wenpeng, "Advanced metering infrastructure," Southern Power System Technology, vol. 3, no. 2, pp. 6-10, 2009.

[11] P. Palensky and D. Dietrich, "Demand side management: Demand response, intelligent energy systems, and smart loads," Industrial Informatics, IEEE Transactions on, vol. 7, no. 3, pp. 381-388, 2011. 
[12] N. G. Paterakis, O. Erdinc, J. P. Catalão, and A. G. Bakirtzis, "Optimum generation scheduling based dynamic price making for demand response in a smart power grid," in Technological Innovation for Collective Awareness Systems, pp. 371-379, Springer, 2014.

[13] S. J. Yu, H. Y. Jin, X. B. Tan, and K. Q. Wang, "An economical scheduling strategy of battery-equipped data server with dynamic-pricing power supply in smart grid," in Advanced Materials Research, vol. 869, pp. 426-431, Trans Tech Publ, 2014.

[14] M. Albadi and E. El-Saadany, "Demand response in electricity markets: An overview," in IEEE Power Engineering Society General Meeting, vol. 2007, pp. 1$5,2007$.

[15] G. Barbose, C. Goldman, and B. Neenan, "A survey of utility experience with real time pricing," 2004.

[16] E. Coffman, M. Garey, D. Johnson, and R. Tarjan, "Performance bounds for level-oriented two-dimensional packing algorithms," SIAM Journal on Computing, vol. 9, Issue 4, pp. 808-826, 1980.

[17] M. Duvall, E. Knipping, M. Alexander, L. Tonachel, and C. Clark, "Environmental assessment of plug-in hybrid electric vehicles," EPRI, July, 2007.

[18] C. Guille and G. Gross, "A conceptual framework for the vehicle-to-grid (v2g) implementation," Energy policy, vol. 37, no. 11, pp. 4379-4390, 2009.

[19] H. Lund and W. Kempton, "Integration of renewable energy into the transport and electricity sectors through v2g," Energy policy, vol. 36, no. 9, pp. 3578-3587, 2008 .

[20] Q. Yang, Z. Bao, W. Yan, and T. Wu, "Smart energy management in microgrid with wind power generators and plug-in electric vehicles," in Plug In Electric Vehicles in Smart Grids, pp. 249-277, Springer, 2015.

[21] W. Su, J. Wang, and J. Roh, "Stochastic energy scheduling in microgrids with intermittent renewable energy resources," Smart Grid, IEEE Transactions on, vol. 5, no. 4, pp. 1876-1883, 2014.

[22] J. G. Kassakian, R. Schmalensee, G. Desgroseilliers, T. D. Heidel, K. Afridi, A. Farid, J. Grochow, W. Hogan, H. Jacoby, J. Kirtley, et al., "The future of the electric grid," Massachusetts Institute of Technology, Tech. Rep, pp. 197-234, 2011.

[23] H. W. Stanton, "Impact of plug-in hybrid vehicles on the electric grid," Oak Ridge National Laboratory, Tennessee, 2006.

[24] J. Song, A. Toliyat, D. Turtle, and A. Kwasinski, "A rapid charging station with an ultracapacitor energy storage system for plug-in electrical vehicles," in Electrical Machines and Systems (ICEMS), 2010 International Conference on, pp. 2003-2007, IEEE, 2010. 
[25] S. Borenstein, "Customer risk from real-time retail electricity pricing: Bill volatility and hedgability," tech. rep., National Bureau of Economic Research, 2006.

[26] Z. Tan, P. Yang, and A. Nehorai, "An optimal and distributed control strategy for charging plug-in electrical vehicles in the future smart grid," in Plug In Electric Vehicles in Smart Grids, pp. 79-106, Springer, 2015.

[27] T. Jiang, Y. Cao, L. Yu, and Z. Wang, "Load shaping strategy based on energy storage and dynamic pricing in smart grid," IEEE Transactions on Smart Grid, vol. 5, Issue 6, pp. 2868-2876, 2014.

[28] L. Bai, G. Xu, and N. D. A. Rahman, "Models for optimal charging scheduling for electric vehicles in smart grid," International Journal of Operational Research, vol. 21, no. 1, pp. 59-83, 2014.

[29] K. Morrow, D. Karner, and J. Francfort, "Plug-in hybrid electric vehicle charging infrastructure review," US Departent of Energy-Vehicle Technologies Program, 2008 .

[30] K. Lebeau, J. Van Mierlo, P. Lebeau, O. Mairesse, and C. Macharis, "Consumer attitudes towards battery electric vehicles: a large-scale survey," International Journal of Electric and Hybrid Vehicles, vol. 5, no. 1, pp. 28-41, 2013.

[31] A. Y. Lam, Y.-W. Leung, and X. Chu, "Electric vehicle charging station placement: Formulation, complexity, and solutions," IEEE Transactions on Smart Grid, vol. 5, Issue 6, pp. 2846-2856, 2013.

[32] M. M. Karbasioun, I. Lambadaris, G. Shaikhet, and E. Kranakis, "Optimal controlling of charging stations for heterogeneous electric vehicles," Submitted to the journal of Computer Communications, 2018.

[33] M. M. Karbasioun, G. Shaikhet, I. Lambadaris, and E. Kranakis, "Asymptotically optimal scheduling of random malleable demands in smart grid," The journal on Discrete Mathematics, Algorithms and Applications (DMAA), vol. 10, no. $02,2018$.

[34] M. M. Karbasioun, I. Lambadaris, G. Shaikhet, and E. Kranakis, "Optimal charging strategies for electrical vehicles under real time pricing," in IEEE International Conference on Smart Grid Communications (SmartGridComm), pp. 746751, IEEE, 2014.

[35] M. Karbasioun, G. Shaikhet, E. Kranakis, and I. Lambadaris, "Power strip packing of malleable demands in smart grid," in IEEE International Conference on Communications (ICC), Budapest, Hungary, 2013.

[36] G. Shaikhet, M. Karbasioun, E. Kranakis, and I. Lambadaris, "Asymptotic convex optimization for packing random malleable demands in smart grid," in IEEE International Conference on Communications (ICC), Budapest, Hungary, 2013.

[37] J. S. Vardakas, N. Zorba, and C. V. Verikoukis, "Performance evaluation of power demand scheduling scenarios in a smart grid environment," Applied Energy, vol. 142, pp. 164-178, 2015. 
[38] N. Ruiz, I. Cobelo, and J. Oyarzabal, "A direct load control model for virtual power plant management," IEEE Transactions on Power Systems, vol. 24, no. 2, pp. 959-966, 2009.

[39] M. H. Albadi and E. El-Saadany, "A summary of demand response in electricity markets," Electric Power Systems Research, vol. 78, no. 11, pp. 1989-1996, 2008.

[40] M. M. Jalali and A. Kazemi, "Demand side management in a smart grid with multiple electricity suppliers," Energy, vol. 81, pp. 766-776, 2015.

[41] M. López, S. de la Torre, S. Martín, and J. Aguado, "Demand-side management in smart grid operation considering electric vehicles load shifting and vehicleto-grid support," International Journal of Electrical Power $\& 6$ Energy Systems, vol. 64, pp. 689-698, 2015.

[42] A.-H. Mohsenian-Rad, V. W. Wong, J. Jatskevich, R. Schober, and A. LeonGarcia, "Autonomous demand-side management based on game-theoretic energy consumption scheduling for the future smart grid," IEEE transactions on Smart Grid, vol. 1, no. 3, pp. 320-331, 2010.

[43] A.-H. Mohsenian-Rad, V. W. Wong, J. Jatskevich, and R. Schober, "Optimal and autonomous incentive-based energy consumption scheduling algorithm for smart grid," in Innovative Smart Grid Technologies (ISGT), 2010, pp. 1-6, IEEE, 2010.

[44] I. Koutsopoulos and L. Tassiulas, "Control and optimization meet the smart power grid: Scheduling of power demands for optimal energy management," in Arxiv preprint: http://arxiv.org/abs/1008.3614v1, 2010.

[45] J. Le Boudec and D. Tomozei, "Satisfiability of elastic demand in the smart grid," in Arxiv preprint: http://arxiv.org/abs/1011.5606v2, 2011.

[46] S. Caron and G. Kesidis, "Incentive-based energy consumption scheduling algorithms for the smart grid," in First IEEE International Conference on Smart Grid Communications (SmartGridComm), 2010.

[47] I. S. Al-Anbagi and H. T. Mouftah, "Qos schemes for charging plug-in electric vehicles in a smart grid environment," in Plug In Electric Vehicles in Smart Grids, pp. 241-265, Springer, 2015.

[48] D. Li and S. K. Jayaweera, "Distributed smart-home decision-making in a hierarchical interactive smart grid architecture," IEEE Transactions on Parallel and Distributed Systems, vol. 26, Issue 1, pp. 75-84, 2015.

[49] I. Koutsopoulos, V. Hatzi, and L. Tassiulas, "Optimal energy storage control policies for the smart power grid," in IEEE International Conference on Smart Grid Communications (SmartGridComm), 2011.

[50] C. Kenyon and E. Rémila, "A near-optimal solution to a two-dimensional cutting stock problem," Mathematics of Operations Research, vol. 25, No. 4, pp. 645-656, 2000 . 
[51] B. Baker, E. Coffman Jr, and R. Rivest, "Orthogonal packings in two dimensions," SIAM Journal on Computing, vol. 9, pp. 846-855, 1980.

[52] A. Lodi, S. Martello, and M. Monaci, "Two-dimensional packing problems: A survey," European Journal of Operational Research, vol. 141, Issue 2, no. 2, pp. 241-252, 2002.

[53] C. Kenyon, "Best-fit bin-packing with random order," in Proceedings of the seventh annual ACM-SIAM symposium on Discrete algorithms (SODA 96), pp. 359364, 1996.

[54] S. Halfin, "Next-fit bin packing with random piece sizes," Journal of applied probability, vol. 26, No. 3, pp. 503-511, 1989.

[55] N. Ntene and J. H. van Vuuren, "A survey and comparison of guillotine heuristics for the 2d oriented offline strip packing problem," Discrete Optimization, vol. 6, no. 2, pp. 174-188, 2009.

[56] A. Steinberg, "A strip-packing algorithm with absolute performance bound 2," SIAM Journal on Computing, vol. 26, no. 2, pp. 401-409, 1997.

[57] C. L. Mumford-Valenzuela, J. Vick, and P. Y. Wang, "Heuristics for large strip packing problems with guillotine patterns: An empirical study," in Metaheuristics: computer decision-making, pp. 501-522, Springer, 2004.

[58] E. Hopper and B. Turton, "An empirical investigation of meta-heuristic and heuristic algorithms for a $2 \mathrm{~d}$ packing problem," European Journal of Operational Research, vol. 128, no. 1, pp. 34-57, 2001.

[59] R. Harren and R. van Stee, "Improved absolute approximation ratios for twodimensional packing problems," in Approximation, Randomization, and Combinatorial Optimization. Algorithms and Techniques, pp. 177-189, Springer, 2009.

[60] I. Schiermeyer, "Reverse-fit: A 2-optimal algorithm for packing rectangles," in AlgorithmsÜESA'94, pp. 290-299, Springer, 1994.

[61] R. Harren, K. Jansen, L. Prädel, and R. Van Stee, "A $(5 / 3+\varepsilon)$-approximation for strip packing," Computational Geometry, vol. 47, no. 2, pp. 248-267, 2014.

[62] K. Jansen and R. Solis-Oba, "Rectangle packing with one-dimensional resource augmentation," Discrete Optimization, vol. 6, no. 3, pp. 310-323, 2009.

[63] E. Hopper and B. Turton, "A review of the application of meta-heuristic algorithms to 2d strip packing problems," Artificial Intelligence Review, Springer, vol. 16, No. 4, pp. 257-300, 2001.

[64] E. Coffman Jr, P. Downey, and P. Winkler, "Packing rectangles in a strip," Acta informatica, Springer, vol. 38, No. 10, pp. 673-693, 2002.

[65] E. Coffman and P. Shor, "Average-case analysis of cutting and packing in two dimensions," European Journal of Operational Research, Elsevier, vol. 44, Issue 2, pp. 134-144, 1990. 
[66] C. Imreh, "Online strip packing with modifiable boxes," Operations Research Letters, Elsevier, vol. 29, Issue 2, pp. 79-85, 2001.

[67] K. Jansen, "Scheduling malleable parallel tasks: an asymptotic fully polynomial time approximation scheme," Algorithmica, vol. 39, Number 1, pp. 59 - 81, 2004.

[68] A. Ranjan, P. Khargonekar, and S. Sahni, "Offline preemptive scheduling of power demands to minimize peak power in smart grids," in Computers and Communication (ISCC), 2014 IEEE Symposium on, pp. 1-6, IEEE, 2014.

[69] A. Ranjan, P. Khargonekar, and S. Sahni, "Tight performance bound for bottom left decreasing height scheduling of preemptable power loads." http://www . cise.ufl.edu/ aranjan/Papers/BLDH.pdf, 2014. Accessed on 2015-08-15.

[70] A. Ranjan, P. Khargonekar, and S. Sahni, "Offline scheduling of power loads in smart grids." http: //www. cise .ufl. edu/ aranjan/Papers/NFDH. pdf, 2014. Accessed on 2015-08-15.

[71] A. Ranjan, P. Khargonekar, and S. Sahni, "Offline first fit decreasing height scheduling of power loads." http://www.cise.ufl.edu/ aranjan/Papers/ FFDH.pdf, 2014. Accessed on 2015-08-15.

[72] I. S. Bayram, G. Michailidis, M. Devetsikiotis, S. Bhattacharya, A. Chakrabortty, and F. Granelli, "Local energy storage sizing in plug-in hybrid electric vehicle charging stations under blocking probability constraints," in Smart Grid Communications (SmartGridComm), 2011 IEEE International Conference on, pp. 7883, IEEE, 2011.

[73] S. M. Ross, Introduction to probability models. Academic press, 2009.

[74] P. Kumar and P. Varaiya, Stochastic systems: estimation, identification and adaptive control. Prentice-Hall, Inc., 1986.

[75] S. Ross, Introduction to Stochastic Dynamic Programming. Academic Press, Inc. Orlando, FL, USA, 1983.

[76] Z. Rosberg, P. Varaiya, and J. Walrand, "Optimal control of service in tandem queues," IEEE Transactions on Automatic Control, vol. 27 , Issue: 3, pp. 600 610, 1982.

[77] M. Alonso, H. Amaris, J. G. Germain, and J. M. Galan, "Optimal charging scheduling of electric vehicles in smart grids by heuristic algorithms," Energies, vol. 7, no. 4, pp. 2449-2475, 2014.

[78] M. S. Kuran, A. Carneiro Viana, L. Iannone, D. Kofman, G. Mermoud, and J.-P. Vasseur, "Smart cities recharged: Improving electrical vehicles recharging by routine-aware scheduling," in Wireless and Mobile Computing, Networking and Communications (WiMob), 2014 IEEE 10th International Conference on, pp. 106-113, IEEE, 2014. 
[79] I. S. Bayram, G. Michailidis, M. Devetsikiotis, and B. Parkhideh, "Strategies for competing energy storage technologies for in dc fast charging stations," in Smart Grid Communications (SmartGridComm), 2012 IEEE Third International Conference on, pp. 1-6, IEEE, 2012.

[80] I. S. Bayram, G. Michailidis, M. Devetsikiotis, and F. Granelli, "Electric power allocation in a network of fast charging stations," IEEE Journal on Selected Areas in Communications, vol. 31, no. 7, pp. 1235-1246, 2013.

[81] P. Van de Ven, N. Hegde, L. Massoulie, and T. Salonidis, "Optimal control of residential energy storage under price fluctuations," in ENERGY 2011 : The First International Conference on Smart Grids, Green Communications and IT Energy-aware Technologies, 2011.

[82] N. Karmarkar and R. Karp, "An efficient approximation scheme for the onedimensional bin-packing problem," in 23rd Annual Symposium on Foundations of Computer Science (SFCS '08), 1982.

[83] I. Koutsopoulos and L. Tassiulas, "Optimal control policies for power demand scheduling in the smart grid," IEEE Journal on Selected Areas in Communications, vol. 30, no. 6, pp. 1049-1060, 2012.

[84] S. Boyd and L. Vandenberghe, Convex optimization. Cambridge University Press, 2004.

[85] C. L. Mumford-Valenzuela, J. Vick, and P. Y. Wang, "Heuristics for large strip packing problems with guillotine patterns: An empirical study," in Metaheuristics: computer decision-making, pp. 501-522, Springer, 2003.

[86] E. K. Burke, G. Kendall, and G. Whitwell, "A new placement heuristic for the orthogonal stock-cutting problem," Operations Research, vol. 52, no. 4, pp. 655$671,2004$.

[87] P. Sánchez-Martín and G. Sanchez, "Optimal electric vehicles consumption management at parking garages," in PowerTech, 2011 IEEE Trondheim, pp. 1-7, IEEE, 2011.

[88] G. Wang, M. Negrete-Pincetic, A. Kowli, E. Shafieepoorfard, S. Meyn, and U. V. Shanbhag, "Dynamic competitive equilibria in electricity markets," in Control and optimization methods for electric smart grids, pp. 35-62, Springer, 2012.

[89] M.-S. Arellano and P. Serra, "A model of market power in electricity industries subject to peak load pricing," Energy Policy, vol. 35, no. 10, pp. 5130-5135, 2007. 


\section{Appendix A}

\section{Proof of Lemma 1 in Chapter 3}

Proof. Suppose that there is a sequence $s_{j}, j=1, \ldots, q$ satisfying $\ell \leq s_{j} \leq r$. Now Consider the following definition: $s^{\prime} \triangleq \frac{1}{q} \sum_{j=1}^{q} s_{j}=\sum_{j=1}^{q} \frac{1}{q} \cdot s_{j}$ (Clearly $\left.\ell \leq s^{\prime} \leq r\right)$. Then $w$ is coverable if and only if there exist an integer value $q$ and a real value $s^{\prime}$ such that $\ell \leq s^{\prime} \leq r$ and $w=q . s^{\prime}$. So we have:

$$
\begin{array}{r}
s^{\prime}=\frac{w}{q} \Longleftrightarrow \ell \leq \frac{w}{q} \leq r \\
\Longleftrightarrow \frac{w}{r} \leq q \leq \frac{w}{\ell} \Longleftrightarrow\left\lceil\frac{w}{r}\right\rceil \leq \frac{w}{\ell}
\end{array}
$$

Therefore the sequences $s_{j}, j=1, \ldots, q$ satisfying $\ell \leq s_{j} \leq r$ exist if and only if $\left\lceil\frac{w}{r}\right\rceil \leq \frac{w}{\ell}$. 


\section{Appendix B}

\section{Proof of Lemma 2 in Chapter 3}

Proof. Instead of using $\sum_{i=1}^{q} s_{i}$, we just use its equivalent value $q \cdot s^{\prime}$, where $s^{\prime}=$ $\frac{1}{q} \sum_{i=1}^{q} s_{i}$. Suppose that there exists a value $v=q \cdot s^{\prime}$ such that $w^{*}<v<w$ which means $q \cdot s^{\prime}>r \cdot\left\lfloor\frac{w}{r}\right\rfloor$ (Note that $q \cdot s^{\prime} \neq w$ because $w$ is not coverable). We know that: $s^{\prime} \leq r$. Combining these two inequality results in: $q>\left\lfloor\frac{w}{r}\right\rfloor$, which implies that:

$$
\left\lceil\frac{w}{r}\right\rceil \leq q
$$

On the other hand $\ell \leq s^{\prime}$, so we have $q \cdot \ell \leq q \cdot s^{\prime}<w$ which results in:

$$
q<\frac{w}{\ell}
$$

Combing the inequalities (B.1) and (B.2) results in $\left\lceil\frac{w}{r}\right\rceil<\frac{w}{\ell}$, which contradicts the fact that $w$ is not coverable ( Lemma 1 in Chapter 3). Therefore the closest point to $w$ which is also smaller than $w$ is $w^{*}=r \cdot\left\lfloor\frac{w}{r}\right\rfloor$. 


\section{Appendix $\mathrm{C}$}

\section{Proof of Lemma 4 in Chapter 3}

Proof. To prove Lemma 4, we will show that for any FSP policy $\theta$ with $P_{\text {max }}^{\theta}$, there exists a filling $\theta^{*}$ with $P_{\max }^{\theta^{*}}$ such that $P_{\max }^{\theta^{*}} \leq P_{\max }^{\theta}$. In $\theta$, first consider an interval $\mathcal{T}=\left[t_{1}, t_{2}\right]$ such that $P(t)=P_{\text {max }}^{\theta} \forall t \in \mathcal{T}$. Now suppose there is a gap in $\mathcal{T}$ such that the length of this gap is more than the length of a narrow rectangle completely placed in $\mathcal{T}$. Then this rectangle can be put in that gap while keeping the $P(t)=P_{\max }^{\theta}$ in $\mathcal{T}$. Therefore we assume that $\mathcal{T}$ doesn't contain this kind of gaps. So in $\theta$ in every interval $\mathcal{T}$ such that $P(t)=P_{\text {max }}^{\theta} \forall t \in \mathcal{T}$ any rearrangement of the narrow rectangles does not increase $P_{\text {max }}^{\theta}$. Now we try to pick a rectangle scheduled in the interval $\mathcal{T}$ (partly or completely) and put it in an unfilled row. This results in a new scheduling $\theta^{\prime}$ with $P_{\max }^{\theta^{\prime}} \leq P_{\max }^{\theta}$. Now we repeat this procedure for $\theta^{\prime}$ and continue doing it until we get a scheduling $\theta^{*}$ in which every row becomes filled (maybe except for the last row), so $\theta^{*}$ is a filling with $P_{\max }^{\theta^{*}} \leq P_{\max }^{\theta}$. 


\section{Appendix D}

\section{Proof of Lemma 5 in Chapter 3}

Proof. With respect to the conditions $0<\ell \leq r<1$ and $\left\lceil\frac{1}{r}\right\rceil>\frac{1}{\ell}$, we conclude that $t=1$ is not coverable. Now define $K_{0}=\left\lfloor\frac{1}{r}\right\rfloor=\left\lfloor\frac{1}{\ell}\right\rfloor$. So we have: $\left(K_{0}+1\right)=\left\lceil\frac{1}{\ell}\right\rceil>\frac{1}{\ell}$, which means: $\left(K_{0}+1\right) \cdot \ell>1$ and hence: $1-K_{0} \cdot \ell<\ell$. The last inequality shows that there is no space to add even the smallest narrow rectangle (i.e. $s_{i}=\ell$ ) to the point $K_{0} \cdot \ell \leq 1$ and since $\ell \leq s_{i}, K_{0}$ is the largest number of rectangles which can fill a row and don't exceed $t=1$. On the other hand, from Lemma 2 in Chapter 3, $Z^{*}=K_{0} \cdot r$ is the largest coverable value in [0,1], i.e. $K_{0} r \leq 1$ and $1-K_{0} r \leq \ell$. Since $s_{i} \leq r, K_{0}$ is the smallest number of rectangles which can fill a row such that there is no space to add a new rectangle to this row. Therefore $K_{0}$ is the exact number of rectangles in each filled row. 